\title{
A THEORY OF SPHERICAL FUNCTIONS. I
}

\author{
BY \\ ROGER GODEMENT
}

\section{INTRODUCTION}

The fact that there exists a close connection between the classical theory of spherical harmonics and that of group representations was first established by $\mathrm{E}$. Cartan and H. Weyl in well known papers; in fact, spherical harmonics arise in a natural way from a study of functions on $G / K$, where $G$ is the orthogonal group in $n$-space and where $K$ consists of those transformations in $G$ under which a given vector is invariant. However, it is obvious that in order to get a theory applying to larger classes of special functions it is necessary to assume that only $K$ is compact, and to study not only functions on $G / K$ but also functions on $G$.

The first nontrivial example was given by V. Bargman in 1947; in his paper [1] on the group $G=S L(2, R)$, Bargman uses a maximal compact subgroup $K$ of $G$ ( $K$ is one-dimensional) and studies functions $\phi(g)$ which, for a given character $\chi$ of $K$, satisfy $\phi\left(k g k^{\prime}\right)=\chi(k) \phi(g) \chi\left(k^{\prime}\right)$. In particular, such functions occur by considering finite- or infinite-dimensional irreducible representations of $G$; these representations have coefficients satisfying the above relation, and from explicit calculations it follows that these functions can be identified in a simple way with particular hypergeometric functions; the case where $\chi(k)=1$ leads to Legendre functions of arbitrary index and to group-theoretical explanations of three important properties of these functions, namely, their differential equation, their representation by integral formulas, and their functional equation (which is connected with the classical addition formulas).

At the same time I. Gelfand and M. Naimark were led to study similar questions by considering irreducible unitary representations of the complex unimodular group $G=S L(n, C)$; if $K$ is a maximal compact subgroup of $G$, then it turns out that some of these representations have coefficients $\phi$ satisfying $\phi\left(k g k^{\prime}\right)=\phi(g)$; these functions have properties entirely similar to those of the Legendre functions; however, they are not very interesting from the point of view of the theory of "special" functions because they can be expressed in closed form in terms of exponential functions (the formulas are very similar to $\mathrm{H}$. Weyl's for the characters of compact groups).

In 1950 very important results were published without proofs by I. Gelfand; in [6] Gelfand considers a (not necessarily compact) Lie group $G$ and a compact subgroup $K$ of $G$, and studies functions $\phi(g)$ satisfying $\phi\left(k g k^{\prime}\right)$

Received by the editors January 26, 1952. 
$=\phi(g)$; those functions which are integrable on $G$ form a normed algebra $A$ under the convolution product on $G$; under the assumption that there exists on $G$ an anti-involution $g \rightarrow \bar{g}$ such that $k^{-1}=\bar{k}$, Gelfand proves that $\boldsymbol{A}$ is commutative; his "spherical functions" are then defined by the following requirements: they are continuous, positive-definite, invariant under $g \rightarrow k g k^{\prime}$, and finally the linear form $f \rightarrow \phi(f)=\int f(g) \phi(g) d g$ must be a homomorphism of $A$ onto the complex field. This property is equivalent to the functional equation

$$
\int \phi\left(k g k^{-1} g^{\prime}\right) d k=\phi(g) \phi\left(g^{\prime}\right),
$$

and implies that spherical functions correspond to "characters" or to maximal ideals of the algebra $\boldsymbol{A}$; Gelfand then applies the general theory of commutative normed algebras to prove a generalization of Bochner's theorem, namely, that every continuous positive-definite function invariant under $g \rightarrow k g k^{\prime}$ is the "continuous sum" of a locally compact set of spherical functions with respect to some positive and bounded measure; this result can be interpreted as a decomposition theorem for unitary representations of $G$ which contain "sufficiently many" vectors invariant under $K$, and proves that the general decomposition theorems due to von Neumann can be considerably improved in special and important situations: more accurately Gelfand's result means that the "exceptional null-sets" of the general theory do not occur under suitable assumptions. On the other hand, Gelfand exhibits differential equations for his spherical functions, and these differential equations of course express that the spherical functions, considered as functions on $G / K$, are eigenfunctions for every differential operator invariant under the motion group of the symmetric Riemann space $G / K$.

Despite their importance, these results are not general enough, mainly for the following two reasons: (1) Gelfand studies only unitary representations of $G$; (2) he studies only functions which are invariant under $g \rightarrow k g k^{\prime}$. It is obvious that not necessarily unitary representations (e.g., finite-dimensional representations) must be taken into consideration, and on the other hand there is no reason (except for convenience!) to consider only the trivial representation $k \rightarrow 1$ of $K$. Actually, it is necessary to consider an arbitrary irreducible representation $k \rightarrow U_{k}$ of $K$ and to study functions $\phi(g)=\operatorname{Tr}[\Phi(g)]$, where $\Phi(g)$ is an operator on the representation space of $k \rightarrow U_{k}$ and where

$$
\Phi(k g h)=U_{k} \Phi(g) U_{h}
$$

for every $k, h \in K$; this of course was done by Bargman for the hyperbolic group. Such functions can be obtained in the following special way. Consider a representation $g \rightarrow T_{0}$ of $G$ on a Banach space $\mathfrak{S}$; denote by $\mathfrak{d}$ the equivalence class of $k \rightarrow U_{k}$, and let $\mathfrak{S}(\mathfrak{d})$ be the set of vectors in $\mathfrak{S}$ which, under 
$k \rightarrow T_{k}$, transform according to $\mathfrak{b}$; then $\mathfrak{S}(\mathfrak{b})$ is a closed subspace and there exists a continuous projection $E(\mathfrak{d})$ of $\mathfrak{S}$ onto $\mathfrak{S}(\mathfrak{d})$. Now assume that $\mathfrak{S}(\mathfrak{d})$ is finite-dimensional; then the function

$$
\phi_{\mathrm{b}}(g)=\operatorname{Tr}\left[E(\boldsymbol{\delta}) T_{g}\right],
$$

where $\operatorname{Tr}$ is the ordinary trace on $\mathfrak{S}$ (defined at least for operators of finite rank), is well defined and has a representation by a function satisfying $\left(^{* *}\right)$.

The general theory of spherical functions consists in studying functions $(* * *)$ corresponding to irreducible representations of $G$. The first general results were recently announced by Harish-Chandra in [20]; HarishChandra's method applies only to semi-simple Lie groups $G$, and rests upon a detailed analysis of algebras of differential operators on $G$ (universal enveloping algebra of the Lie algebra of $G$ ) and a proof of the fact that every irreducible representation of $G$ has analytic coefficients (this is necessary in order to deduce "integral" properties from "infinitesimal" ones). The most important results announced by Harish-Chandra are about as follows: (1) every irreducible representation $\mathfrak{D}$ of $G$ contains every irreducible representation $\mathfrak{b}$ of $K$ a finite number of times only [and this number $n(\mathfrak{b}, \mathfrak{D})$ has a finite upper bound $n(\delta)$ when $\mathfrak{D}$ varies], so that spherical functions can be defined; (2) these spherical functions are analytic and determine up to equivalence the corresponding irreducible representations of $G ;(3)$ if $\delta$ is one-dimensional, then every spherical function $\phi_{0}$ can be represented by an integral formula generalizing those of Bargman and Gelfand-Naimark; similar but less precise results still hold for arbitrary $\delta$; (4) every irreducible representation of $G$ corresponds to an algebraically irreducible representation of its Lie algebra.

Our purpose in the present paper is to prove that a systematic use of functional analysis (normed algebras and so on) leads to: (1) elementary proofs of some of Harish-Chandra's results, in particular the fact that supD $n(\delta, \mathfrak{D})<+\infty ;(2)$ new results; (3) an extension of some of HarishChandra's results to not necessarily semi-simple groups (in particular, to all euclidean motion groups, which are important because they lead to generalized Bessel's functions). The content of our paper is as follows.

In $\$ 1$ we essentially prove the theorem that $(* * * *)$

$$
\sup _{D} n(D, D)<+\infty \text {; }
$$

our method is very simple: we write $G=K \cdot N$ where $N$ is a subgroup which, for semi-simple $G$, is solvable and which, for euclidean motion groups, is abelian and invariant; then we verify that $\sup n(d, \mathfrak{D})<+\infty$ when $\mathfrak{D}$ runs over the set of (not necessarily irreducible) representations of $G$ "induced" by one-dimensional representations of $N$ (this step of the proof is trivial, and inspired by Gelfand and Naimark); next we prove that these induced representations are "in sufficiently great number," and finally $(* * * *)$ fol- 
lows by using a theorem due to I. Kaplansky and asserting that, if an associative algebra has "sufficiently many" representations whose dimensions do not exceed a given number $n$, then every irreducible representation of this algebra has dimension $\leqq n$.

In $\S 2$, we use the assumption that $n(\mathfrak{D}, \mathfrak{D})<+\infty$ in order to prove that every irreducible representation of $G$ is more or less equivalent to the natural representation of $G$ on $A / \mathfrak{m}$, where $A$ is some "group algebra" and where $\mathfrak{m}$ is some regular maximal left ideal in $\boldsymbol{A}$; this fact was known for abelian and for compact groups only (and in this case is trivial).

In $\$ 3$ we study spherical functions (under the same assumption as in $\$ 2$ ) from the point of view of their functional properties. The basic result is equivalent to the following one: let $L^{\circ}(G)$ be the space of continuous functions with compact carrier on $G$ which are invariant under $g \rightarrow k g k^{-1} ; L^{\circ}(G)$ is an algebra under convolution product; now let $\phi$ be a continuous function on $G$, satisfying $\phi\left(k g k^{-1}\right)=\phi(g)$, and define $\phi(f)=\int f(g) \phi(g) d g$ for every $f \in L^{\circ}(G)$; then $\phi$ is proportional to a spherical function if and only if: (a) $\phi$ grows "not too fast" at infinity; (b) there exists a finite-dimensional irreducible representation $f \rightarrow T_{f}$ of $L^{\circ}(G)$ such that $\phi(f)$ is proportional to $\operatorname{Tr}\left(T_{f}\right)$ for every $f \in L^{\circ}(G)$. From this it follows that spherical functions are very similar to the characters of abelian and compact groups (actually, these characters are special instances of spherical functions!); in particular, the spherical function $\left({ }^{* * *}\right)$ satisfies the functional equation $\left({ }^{*}\right)$ if and only if $b$ occurs once only in the given representation of $G$ (but we do not know functional equations for arbitrary spherical functions). Finally we give integral formulas for spherical functions in the case of euclidean motion groups.

In $\S 4$ we study the differential properties of spherical functions. By using the classical Bernstein theorem on elliptic operators we first give a very simple proof of the fact that every irreducible representation of $G$ has analytic coefficients, from which the analyticity of spherical functions follows. Now consider the algebra $U^{\circ}(G)$ of differential operators on $G$ which commute with all translations $g \rightarrow k g g^{\prime}, k \in K, g^{\prime} \in G$, and for a given analytic function $\phi$ invariant under $g \rightarrow k g k^{-1}$ define $\phi(X)=X \phi(e)$ for every $X$ $\in U^{\circ}(G)$; then $\phi$ is proportional to a spherical function if and only if $\phi$ grows not too fast at infinity and if $X \rightarrow \phi(X)$ is proportional to the "character" of a finite-dimensional irreducible representation of $U^{\circ}(G)$. The analogy between this result and that of $\S 3$ is striking, and actually our proof of the "infinitesimal" result consists in proving (by Hahn-Banach's theorem in spaces of "distributions") that it is equivalent to the "integral" one. For spherical functions satisfying the functional equation $\left(^{*}\right)$ the above result takes on a simpler form, namely, that $\phi$ is an eigenfunction of every $X$ $\in U^{\circ}(G)$. Finally we give a very simple proof of the fact that irreducible representations of $G$ lead to algebraically irreducible representations of its Lie 
algebra - this result (which of course is the "infinitesimal" theorem corresponding to the "integral" result of $\$ 2$ ) is important because it proves that the purely algebraic results published by Harish-Chandra in [19] can be used to study the representations from the integral point of view.

In proving the basic result of $\S 1$ we have shown that if $G$ contains a solvable connected group $N$, then every finite-dimensional irreducible representation of $G$ is contained in a representation induced by some one-dimensional representation of $N$. We prove in an appendix that the above remark can be used in order to get a complete classification of finite-dimensional representations of the "classical groups"; our method of course does not lead to new results but is simpler than those of E. Cartan and H. Weyl, and furthermore is purely integral [ $\mathrm{H}$. Weyl's method of characters is purely integral too-although it rests upon integration formulas which cannot be proved without using "infinitesimal" arguments-but it applies to compact and to complex groups only; our method works for all (compact or not, complex or real) classical groups].

In this paper, we are concerned with representations on arbitrary Banach spaces; in a second paper we shall study more closely the unitary representations on Hilbert spaces, in order to prove that the general decomposition theorems (see [15]) can be stated in much better terms under the assumption that $\left({ }^{* * *}\right)$ holds. The method consists in considering, for a given irreducible representation $\delta$ of $K$, the algebra $L^{\circ}(\mathfrak{b})$ of continuous functions with compact carrier on $G$ which have the form $\operatorname{Tr}[\phi(g)]$ where $\Phi$ satisfies $\left({ }^{* *}\right)$ for the given $\delta$, and in observing: (a) that $L^{\circ}(\mathfrak{d})$ is an algebra with an involution whose irreducible unitary representations have finite and bounded dimensions (if $\mathfrak{d}$ is the one-dimensional representation $k \rightarrow 1$, then $L^{\circ}(\mathfrak{d}$ ) is identical - up to completion with respect to the $L^{1}$-norm-with the algebra $\boldsymbol{A}$ of Gelfand, which is commutative); (b) that there exists a one-to-one correspondence between the irreducible unitary representation of $G$ in which o does occur and the irreducible unitary representations of $L^{\circ}(\mathfrak{d})$. By using a very simple argument explained on p. 79 of [15] the above properties enable us to throw out of the decomposition theorems all exceptional null sets which necessarily occur in general groups, to define in a natural way locally compact "dual objects," and to prove topological uniqueness theorems. In other words, the basic results of the harmonic analysis on abelian groups can be extended without any pathological feature to a much larger class of groups, and this is interesting not only from a theoretical point of view but also because this class includes practically all groups whose importance is obvious in "classical" mathematics, namely, the abelian groups, the compact groups, the semisimple Lie groups, and the euclidean motion groups. Therefore it may be hoped that the theory of group representations is now able to provide new results and new interpretations of known results in the most classical parts 
of analysis; this, in the author's opinion, is the ultimate purpose of this theory.

\section{Preliminary Definitions AND RESUlts}

Throughout this paper we shall denote by $G$ a locally compact unimodular group and by $K$ a compact subgroup of $G$. We shall of ten use the following notation: if $X$ denotes a group, then $x$ will denote a generic element of $X$.

We shall denote by $\boldsymbol{M}(G)$ [resp. $\boldsymbol{L}(G)$ ] the space of all measures [resp. continuous functions] with compact carrier on $G$. The convolution product of two measures $\alpha, \beta \in \boldsymbol{M}(G)$ will be denoted by $\alpha \beta$ (so that we omit the ${ }^{*}$ ); see [29] and [13] for the main definitions and results; if $\epsilon_{g}$ denotes the measure on $G$ given by $\theta \rightarrow \theta(g), \theta \in L(G)$, then it is easy to see that

$$
\alpha \beta=\iint \epsilon_{g^{\prime} g^{\prime \prime}} \cdot d \alpha\left(g^{\prime}\right) d \beta\left(g^{\prime \prime}\right)
$$

-of course, (1) is a "weak" integral. We shail always identify a measure $\alpha \in \boldsymbol{M}(K)$ on $K$ with the measure $\alpha \in \boldsymbol{M}(G)$ on $G$ given by $\theta \rightarrow \int \theta(k) d \alpha(k)$; by (1) it is clear that we get an isomorphism of the algebra $\boldsymbol{M}(K)$ into the algebra $\boldsymbol{M}(G)$.

We shall choose once and for all a Haar measure $d g$ on $G$, and we shall always identify every (for instance, continuous) function $\theta(g)$ with the corresponding measure $\theta(g) d g$. In the same way, every continuous function on $K$ will be identified with a measure on $K$, hence with a measure on $G$. It is known and obvious that $\boldsymbol{L}(G)$ is a two-sided ideal in $\boldsymbol{M}(G)$.

By a representation of $G$ on a Banach space $\mathfrak{S}$ we shall mean a homomorphism $g \rightarrow T_{g}$ of $G$ into the group of nonsingular bounded operators on $\mathfrak{S}$, with the requirement that, for every $a \in \mathfrak{S}$, the mapping $g \rightarrow T_{0} a$ of $G$ into $\mathfrak{S}$ is (strongly) continuous. For every compact set $A \subset G$ and every $\boldsymbol{a} \in \mathfrak{S}$, the set of vectors $T_{o} \boldsymbol{a}, g \in A$, is therefore compact, hence bounded in $\mathfrak{S}$; by well known arguments (Banach-Steinhaus theorem) this proves that the function

$$
\rho(g)=\left\|T_{g}\right\|
$$

is bounded on every compact set. It is obvious that $\rho(g)$ is lower semi-continuous on $G$, and satisfies

$$
\rho\left(g^{\prime} g^{\prime \prime}\right) \leqq \rho\left(g^{\prime}\right) \rho\left(g^{\prime \prime}\right)
$$

for every two $g^{\prime}, g^{\prime \prime} \in G$; such a function will be called a semi-norm on $G$. If, for a given semi-norm $\rho$ and every $f \in L(G)$, we set

$$
\|f\|_{\rho}=\int|f(g)| \rho(g) d g
$$


then (3) implies $\left\|f^{\prime} f^{\prime \prime}\right\|_{\rho} \leqq\left\|f^{\prime}\right\|_{\rho} \cdot\left\|f^{\prime \prime}\right\|_{\rho}$; hence the space $\boldsymbol{A}_{\rho}(G)$ of absolutely integrable functions with respect to $\rho(g) d g$ can be considered in a natural way as a complete normed algebra under the convolution product. Of course, $\boldsymbol{L}(G)$ is everywhere dense in $A_{\rho}(G)$-by the very definition of integrable functions.

Every representation $g \rightarrow T_{o}$ of $G$ can be extended to a representation $\alpha \rightarrow T_{\alpha}$ of $M(G)$, given by

$$
T_{\alpha}=\int T_{g} \cdot d \alpha(g)
$$

if $d \alpha(g)=f(g) d g$ with $f \in L(G)$, we shall write $T_{f}$ instead of $T_{\alpha}$. It is obvious from (2) that $\left\|T_{f}\right\| \leqq\|f\|_{\rho}$; hence $f \rightarrow T_{f}$ can be extended to the whole of $\boldsymbol{A}_{\rho}(G)$. Conversely, let $f \rightarrow T_{f}$ be a representation of $\boldsymbol{A}_{\rho}(G)$ on a Banach space $\mathfrak{S}$, and assume that $\left\|T_{f}\right\| \leqq\|f\|_{\rho}$ for every $f \in A_{\rho}(G)$; then the given representation of $\boldsymbol{A}_{\rho}(G)$ is generated, by the above process, by a representation of $G$ on $\mathfrak{S}$, provided the set of vectors $T_{f} a\left[f \in A_{\rho}(G), a \in \mathfrak{S}\right]$ span the whole of $\mathfrak{S}\left({ }^{1}\right)$.

Concerning the concept of "irreducibility," we shall use three different definitions.

Definition 1. Let $A$ be an arbitrary associative algebra over a field $\Omega$, and let $x \rightarrow T_{x}$ be a linear representation of $A$ on an arbitrary vector spáce $\mathfrak{S}$ over $\Omega$; then the given representation is said to be algebraically irreducible if $\mathfrak{S}$ contains no nontrivial subspace invariant under the $T_{x}$.

Definition 2. Let $A$ be an associative algebra over the complex field and let $x \rightarrow T_{x}$ be a linear representation of $\boldsymbol{A}$ by bounded operators on a Banach space $\mathfrak{S} ;$ then the given representation is said to be completely irreducible if every bounded operator on $\mathfrak{S}$ is a strong limit $\left.{ }^{2}\right)$ of operators $T_{x}$.

DeFINITION 3. With the notation of Definition 2, the representation $x \rightarrow T_{x}$

(1) This can be proved in the following way. If $f \in A_{\rho}(G)$ and $s \in G$, then it is obvious that $\boldsymbol{\epsilon}_{s} f \in \boldsymbol{A}_{\rho}(G)$ and that $\left\|\epsilon_{s} f\right\|_{\rho} \leqq \rho(s) \cdot\|f\|_{\rho}$. Furthermore, for every compact neighborhood $V$ of $s$ in $G$, denote by $g_{V}$ a positive continuous function on $G$ vanishing outside $V$ and such that $\int g_{V}(x) d x=1$; then it is clear that $g_{V} f$ converges strongly in $\boldsymbol{A}_{\rho}(G)$ to $\epsilon_{s} f$ for every $f \in \boldsymbol{A}_{\rho}(G)$; furthermore we have $\left\|g_{V}\right\|_{\rho} \leqq \sup _{t \in V} \rho(t)$ so that by setting $\rho^{*}(s)=\inf _{V} \sup _{t \in V} \rho(t)(<+\infty)$ we see that $\left\|g_{V}\right\|_{\rho} \leqq \rho^{*}(s)+\epsilon$ provided $V$ is small enough. Now consider a continuous representation $f \rightarrow T_{f}$ on a Banach space $\mathfrak{W}$, and assume the set $\mathfrak{S}_{0}$ of vectors $\sum T_{f_{i}} \boldsymbol{a}_{i}\left[f_{i} \in A_{\rho}(G) ; a_{i} \in \mathfrak{S}\right]$ is everywhere dense in $\mathfrak{W}$. If $\boldsymbol{b}=\sum T_{f_{i}} \boldsymbol{a}_{i}$ is a vector in $\mathfrak{S}_{0}$ and if $s \in G$, then by the above remark we see that $\sum T_{o f_{i}} a_{i}=T_{o V} b$ converges to $\sum T_{\epsilon_{g} f_{i}} a_{i}$ when $V$ "converges to $s$," so that $\sum T_{f_{i}} a_{i}=0$ implies $\sum T_{\epsilon_{s} f_{i}} a_{i}=0$; hence we may define an operator $T_{s}$ on $\mathfrak{S}_{0}$ by $T_{s} \sum T_{f_{i}} a_{i}$ $=\sum T_{\epsilon_{s} f_{i}} a_{i}$; furthermore if $b \in \mathfrak{S}_{0}$, we have seen that $T_{s} b=\lim T_{\theta_{V}} b$ so that $\left\|T_{s} b\right\|=\lim \left\|T_{o_{V}} b\right\|$ $\leqq K \cdot \rho^{*}(s)\|b\|$; hence $T_{s}$ is continuous so that-since $\mathfrak{S}_{0}$ is everywhere dense in $\mathfrak{E}$ - we may extend it to a bounded operator on $\mathfrak{S}$; of course $s \rightarrow T_{s}$ is a representation of $G$ (the fact that $s \rightarrow T_{8} b$ is continuous for every $b$ follows from the fact that $\epsilon_{s} f$ depends continuously on $s$ for every $f \in A_{\rho}(G)$ ). It remains to prove that $T_{f}=\int T_{s} \cdot f(s) d s$, which is easy by using the fact that we have, in $A_{\rho}(G), g f=\int \epsilon_{s} f \cdot g(s) d s$ (strong integral). Toa.

(2) We recall that $T$ converges strongly to $T_{0}$ if, for every $a \in \mathfrak{S}, T a$ converges strongly to 
is said to be topologically irreducible if $\mathfrak{S}$ contains no nontrivial invariant closed subspace.

We shall use Definitions 2 and 3 for group representations by considering the corresponding representations of the algebra $L(G)$.

Concerning the connections between these three kinds of irreducibility, it is obvious by Burnside's theorem that they are equivalent for finite-dimen. sional representations. In the general case, it is obvious that complete irreducibility implies topological irreducibility. By the "density theorem of von Neumann" $[27$, p. $397 ; 15$, p. 99] it is known that, for unitary representations on Hilbert spaces, topological irreducibility implies (hence is equivalent to) complete irreducibility $\left(^{(}\right)$.

It is obvious that every completely irreducible representation of a commutative algebra or group is one-dimensional; this is still true for topologically irreducible bounded representations of abelian groups ("bounded" means: $\left\|T_{\theta}\right\|=1$ ), and the proof of this is by no means trivial-it essentially uses the spectral theorem of Beurling (see [14]); but we do not know whether this result still holds for arbitrary topologically irreducible representations! This is the essential reason for which we shall study completely irreducible representations only. In this connection, the following lemma will be an essential tool in our proofs $\left({ }^{4}\right)$ :

Lemma 1. Let $\boldsymbol{A}$ be an associative algebra over the complex field, and let $n$ be a finite integer. Assume that $\boldsymbol{A}$ has sufficiently many linear representations whose dimensions are $\leqq n$. Then every completely irreducible representation of $\boldsymbol{A}$ has dimension $\leqq n$.

Proof. Denote by $\boldsymbol{M}_{n}$ the algebra of all $n \times n$ matrices; it is known [23] that there exists a noncommutative polynomial $P\left(x_{1}, \cdots, x_{r}\right)$ such that:

$\left(\mathrm{P}_{1}\right): P\left(A_{1}, \cdots, A_{r}\right)=0$ whenever $A_{1}, \cdots, A_{r} \in M_{n}$;

$\left(\mathrm{P}_{2}\right)$ : there exist $A_{1}, \cdots, A_{r} \in M_{n+1}$ such that $P\left(A_{1}, \cdots, A_{r}\right) \neq 0$;

$\left(\mathrm{P}_{3}\right): P\left(x_{1}, \cdots, x_{r}\right)$ depends linearly on every $x_{i}$.

Since $\boldsymbol{A}$ has a "separating" set of representations whose dimensions are $\leqq n$, it is clear that $P\left(x_{1}, \cdots, x_{r}\right)=0$ whenever $x_{1}, \cdots, x_{r} \in A$; hence if $x \rightarrow T_{x}$ is a completely irreducible representation of $A$ on some Banach space $\mathfrak{S}$, we shall have $P\left(T_{x_{1}}, \cdots, T_{x_{r}}\right)=0$ identically; let then $A_{1}, \cdots, A_{r}$ be arbitrary bounded operators on $\mathfrak{S}$; approximating $A_{1}$ by operators $T_{x}$ and using $\left(\mathrm{P}_{3}\right)$ we get $P\left(A_{1}, T_{x_{2}}, \cdots, T_{x_{r}}\right)=0$; approximating $A_{2}$ we then get $P\left(A_{1}, A_{2}, T_{x_{z}}, \cdots, T_{x_{r}}\right)=0$, and finally we see that $P\left(A_{1}, \cdots, A_{r}\right)=0$; assume then that $\mathfrak{S}$ contains an $(n+1)$-dimensional subspace $\mathbb{E}$, and denote by $A_{1}, \cdots, A_{r}$ arbitrary operators on $\mathbb{E}$; since $\mathbb{E}$ is finite-dimensional, every

(3) This is true more generally for representations $x \rightarrow T_{x}^{*}$ on Hilbert spaces whenever it is known that the set of $T_{x}$ is invariant under $T \rightarrow T^{*}$.

(4) Special cases of Lemma 1 were published by I. Kaplansky; see for instance [24] for the case of unitary representations. 
$A_{i}$ is induced by some bounded operator on $\mathfrak{E}$; hence $P\left(A_{1}, \cdots, A_{r}\right)=0$, which contradicts $\left(\mathrm{P}_{2}\right)$, q.e.d.

The following lemma is due to J. Dieudonné:

LEMMA 2. Every algebraically irreducible representation of an algebra $\boldsymbol{A}$ by bounded operators on a Banach space $\mathfrak{S}$ is completely irreducible.

Proof. Let $\boldsymbol{M}$ be the set of all endomorphisms of $\mathfrak{S}$ which commute with every $T_{x}$ (the elements of $\boldsymbol{M}$ are not necessarily bounded operators); we first prove that $M$ reduces to scalar operators. In fact, choose a nonvanishing vector $\mathbf{a} \in \mathfrak{S}$ and for $U \in M$ define

$$
\|U\|=\inf \left\|T_{x}\right\|
$$

where $x$ runs over the elements such that $T_{x} \boldsymbol{a}=U \boldsymbol{a}$ (such $x$ do exist because the set of vectors $T_{x} a$ is the whole of $\mathfrak{S}$ ). By trivial computations it is immediately seen that $\boldsymbol{M}$ becomes a normed algebra (i.e., $\|U+V\| \leqq\|U\|+\|V\|$, $\|\lambda \cdot U\|=|\lambda| \cdot\|U\|,\|U V\| \leqq\|U\| \cdot\|V\|)$; but $\boldsymbol{M}$ is a field (Schur's lemma), hence reduces to the complex numbers (Gelfand-Mazur's theorem $\left.{ }^{5}\right)$ ) which proves our assertion. Now we may apply a theorem due to Jacobson [22] and asserting that for every endomorphism $A$ of $\mathfrak{S}$ and every finite set of vectors $\mathbf{a}_{1}, \cdots, \boldsymbol{a}_{n}$ there exists some $x \in A$ such that $T_{x} \boldsymbol{a}_{i}=A \boldsymbol{a}_{i}$ for $1 \leqq i \leqq n$; this obviously proves Lemma 2 (and even somewhat more!).

\section{The MAIN THEOREM ON SEMI-SIMPLE GROUPS}

1. The algebras $L(\mathfrak{d})$. We consider the given group $G$ and its compact subgroup $K$. We shall denote by $\delta$ an irreducible representation of $K$; if $\chi$ is the character of $\mathfrak{b}$ in the classical sense, and if $n(\mathfrak{b})$ is the dimension of $\mathfrak{b}$, then it is well known ("orthogonality relations") that $\chi \chi=n(\mathfrak{d})^{-1} \chi$ (convolution product). We shall denote by $\chi_{\delta}$ the function $n(\delta) \cdot \chi$, so that we have the more easily manageable relation

$$
\chi_{\triangleright} x_{b}=\chi_{\triangleright} .
$$

Now, since we identify functions on $K$ with measures on $G$, we may consider the set $L(\delta)$ of those $f \in L(G)$ which satisfy $\left(^{(6)}\right.$

$$
\bar{\chi}_{\triangleright} f=f \bar{\chi}_{b}=f ;
$$

clearly, $L(\mathfrak{d})$ is a subalgebra of $L(G)$, and $f \rightarrow \bar{\chi}_{\triangleright} f \bar{\chi}_{\mathrm{b}}$ is a projection of $L(G)$ onto $L(\mathfrak{d})$.

Now let $g \rightarrow T_{g}$ be a representation of $G$ on a Banach space $\mathfrak{S}$, and define

$$
E(\S)=\int T_{k} \cdot \bar{\chi}_{\mathfrak{b}}(k) d k
$$

(5) Observe that Gelfand-Mazur's theorem (whose proof uses Liouville's theorem only) holds even in noncommutative and noncomplete normed algebras.

(6) Equation (7) is equivalent to $\int f(k x) \chi_{\mathfrak{b}}(k) d k=\int f(x k) \chi_{\mathfrak{b}}(k) d k=f(x)$ for every $x \in G$. 
by $T_{\alpha \beta}=T_{\alpha} T_{\beta}$ and (6) we see that $E(\delta)$ is a continuous projection of $\mathfrak{S}$ onto a closed subspace $\mathfrak{S}(\mathfrak{D})$; it is easy to see that $\mathfrak{S}(\delta)$ is the set of vectors which, under $k \rightarrow T_{k}$, transform according to $\mathfrak{d}$. (A vector $\mathbf{x} \in \mathfrak{S}$ belongs to $\mathfrak{S}(\mathfrak{d})$ if and only if the vectors $T_{k} \mathbf{x}$ span a finite-dimensional subspace and if the representation $k \rightarrow T_{k}$ of $K$ on this subspace decomposes into irreducible representations which belong to $\delta$.)

If $\operatorname{dim} \mathfrak{S}(\mathfrak{d})=p \cdot \operatorname{dim}(\mathfrak{d})$, we shall say that $\mathfrak{d}$ is contained $p$ times in the given representation of $G$; then the representation $k \rightarrow T_{k}$ of $K$ on $\mathfrak{S}(\delta)$ decomposes into $p$ irreducible representations $\left({ }^{7}\right)$, each of which belongs to $\delta$.

It is clear that we have

$$
E(\mathfrak{b}) T_{f} E(\mathfrak{b})=T_{g} \quad \text { where } \quad g=\bar{\chi}_{\mathfrak{\delta}} f \bar{\chi}_{\mathfrak{\delta}}
$$

for every $f \in L(G)$; hence $\mathfrak{S}(\delta)$ is invariant under $T_{f}$ whenever $f \in L(\delta)$ and if we denote by $\widetilde{T}_{f}$ the operator induced on $\mathfrak{S}(\delta)$ by $T_{f}$, we get a representation $f \rightarrow \tilde{T}_{f}$ of $L(\mathfrak{D})$ on $\mathfrak{S}(\mathfrak{D})$. It is important to observe that, for $f \in L(\mathfrak{D})$, $T_{f}=0$ is equivalent to $\widetilde{T}_{f}=0$.

Lemma 3. Assume the representation $g \rightarrow T_{g}$ of $G$ on $\mathfrak{S}$ is completely irreducible; then, for every $\delta$, the representation $f \rightarrow \widetilde{T}_{f}$ of $L(\mathfrak{D})$ on $\mathfrak{E}(\mathfrak{D})$ is completely irreducible too.

Proof. Let $A$ be a bounded operator on $\mathfrak{S}(\delta)$; extend it to $\mathfrak{S}$ by the condition that $A a=A E(\delta)$ a for every $a \in \mathfrak{S}$; by our assumption, we can find functions $\left(^{8}\right) f_{i} \in L(G)$ such that $T_{f_{i}}$ converges strongly on $\mathfrak{S}$ to $A$; setting $g_{i}=\bar{\chi}_{\delta} f_{i} \bar{\chi}_{\delta}$, it is clear that $g_{i} \in L(\delta)$ and that $\widetilde{T}_{g_{i}}$ converges strongly to $A$ on $\mathfrak{S}(\mathfrak{D})$, q.e.d.

REMARK 1. Similar results hold for algebraically irreducible and for topologically irreducible representations.

REMARK 2. See a special case of Lemma 3 in Gelfand [6].

DEFINITION 4. Let $\Omega$ be a set of representations of $G ; \Omega$ is said to be complete if, for every nonvanishing function $f \in L(G)$, there exists some member $T$ of $\Omega$ such that $T_{f} \neq 0$.

LEMMA 4. Let $\Omega$ be a complete set of representations of $G$; for a given $\delta$ and a given finite integer $p$, assume that $\delta$ is contained at most $p$ times in every member of $\Omega$; then $\mathfrak{d}$ is contained at most $p$ times in every completely irreducible representation of $G$.

Proof. We know that, for $f \in L(\delta), T_{f}=0$ is equivalent to $\widetilde{T}_{f}=0$; hence the representations $f \rightarrow \tilde{T}_{f}$ of $L(\delta)$ associated with the various members of $\Omega$ form a complete set of representations of $L(\delta)$, whose dimensions are $\leqq n$

$\left({ }^{7}\right)$ Strictly speaking this is true for $p<+\infty$ only; in the general case this may be false (if this were true, then every Banach space would admit of at least one basis: take $K=e$ !).

${ }^{(8)}$ Notation $f_{i}$ does not mean that $f_{i}$ is a sequence; see N. Bourbaki, Topologie générale, Chap. I, where the canonical language is explained. 
$=p \cdot \operatorname{dim}(\delta) ;$ hence $($ Lemma 1$)$ every completely irreducible representation of $L(\delta)$ has a dimension $\leqq n$, so that Lemma 4 follows from Lemma 3 .

\section{Applications to semi-simple Lie groups.}

Lemma 5. Let $G$ be a connected semi-simple Lie group with a faithful (finite-dimensional) representation $\left({ }^{9}\right)$; then $G$ has a complete set of finite-dimensional irreducible representations.

Proof. Given a representation $g \rightarrow T_{g}$ on a vector space $\mathfrak{S}$, we call coefficient of that representation every function $\theta(g)=\left(T_{o} a, a^{\prime}\right)$ where $a$ is some vector in $\mathfrak{S}$ and $\boldsymbol{a}^{\prime}$ is some vector in the conjugate space $\mathfrak{S g}^{\prime}$. Denote by $C_{f}$ the set of linear combinations of coefficients of finite-dimensional irreducible representations of $G$; by the complete reducibility, it is clear that $C_{f}$ contains all coefficients of all finite-dimensional representations of $G$; since $G$ has a faithful representation, we see that

$$
\theta\left(g^{\prime}\right)=\theta\left(g^{\prime \prime}\right) \text { for every } \theta \in C_{f} \text { implies } g^{\prime}=g^{\prime \prime} ;
$$

on the other hand, the product of any two coefficients is still a coefficient (tensor product of representations!), and if $\theta \in C_{f}$, we have also $\bar{\theta} \in C_{f}$ (conjugate imaginary representations!); by (10) and the Stone-Weierstrass approximation theorem [2], we thus see that every continuous function on $G$ can be approximated uniformly on every compact set by members of $C_{f}$; hence if $f \in L(G)$ does not vanish, there exists some $\theta \in C_{f}$ such that $\int f(g) \theta(g) d g \neq 0$, and since $\left(T_{f} a, a^{\prime}\right)=\int\left(T_{o} a, a^{\prime}\right) f(g) d g$, Lemma 5 is proved.

THEOREM 1. Let $G$ be a semi-simple connected Lie group with a faithful representation; for $a$ given $\mathfrak{d}$ and a given $p$, assume $\mathfrak{d}$ is contained at most $p$ times in every finite-dimensional irreducible representation of $G$; then $b$ is contained at most $p$ times in every infinite-dimensional completely irreducible representation of $G$.

Proof. Trivial by Lemmas 4 and 5.

Corollary. Let $G=S L(2, C), K=S U(2)$; then every irreducible representation of $K$ occurs at most once in every completely irreducible representation of $G$.

Proof. For every irreducible representation $\delta$ of $K$, there exists a (complex analytic) finite-dimensional irreducible representation $\mathfrak{D}$ of $G$ whose restriction to $K$ is $b$; in addition, every finite-dimensional irreducible representation of $G$ is a tensor product $\mathfrak{D}^{\prime} \otimes \mathfrak{D}^{\prime \prime}$ (this is true for every complex semi-simple group); hence every such representation reduces on $K$ to a tensor product $\mathfrak{d}^{\prime} \otimes \mathfrak{d}^{\prime \prime}$; but it is known (Clebsch-Gordan formula; see [30, p. 115]) that

( $\left.{ }^{9}\right)$ We recall that, for semi-simple connected groups, the existence of a faithful representation is equivalent to that of sufficiently many finite-dimensional representations, and implies that the center of $G$ is finite; see, e.g., Harish-Chandra [18]; we also recall that every complex semi-simple group has a faithful representation. 
$\mathfrak{b}^{\prime} \otimes \mathfrak{d}^{\prime \prime}$ decomposes into inequivalent representations of $K$, so that the assumption of Theorem 1 is satisfied with $p=1$, q.e.d.

Of course, the above corollary can be verified by explicit constructions (at least for unitary representations); see Harish-Chandra [17] and GelfandNaimark [9].

Another corollary of Theorem 1 or Lemma 5 is as follows: let $K$ be a compact subgroup of a connected semi-simple Lie group $G$; let $G_{0}$ be the set of $g \in G$ such that $T_{o}=1$ for every finite-dimensional representation $T$ of $G$; let $k \rightarrow U_{k}$ be an irreducible representation of $K$; then $U$ is contained in some finite-dimensional irreducible representation of $G$ if and only if $U_{k}=1$ for every $k \in K \cap G_{0}$. In fact, if we consider $G / G_{0}$ instead of $G$, we may assume that $G_{0}$ reduces to the identity, i.e., that $G$ has sufficiently many representations (which is equivalent to the existence of a faithful representation); now if $k \rightarrow U_{k}$ were contained in no finite-dimensional representation of $G$, then by Lemma 5 we would have $L(\mathfrak{d})=0$, where $\delta$ denotes $k \rightarrow U_{k}$, hence we would have $\bar{\chi}_{b} f \bar{\chi}_{b}=0$ for every $f \in \boldsymbol{L}(G)$, hence also $\bar{\chi}_{\triangleright} \alpha \bar{\chi}_{\delta}=0$ for every $\alpha \in \boldsymbol{M}(G)$, hence $\bar{\chi}_{b}=0$, which is impossible. The above result is classical for compact $G$ (see A. Weil's book); for semi-simple $G$ it was proved by Harish-Chandra in [18]. Of course, the semi-simplicity assumption is needed in order to ensure that $k \rightarrow U_{k}$ is contained in some irreducible representation of $G$-which means that our result extends to arbitrary $G$ with obvious modifications.

Theorem 1 proves that a sufficiently detailed analysis of finite-dimensional representations yields information about infinite-dimensional ones. We want now to prove that even the study of finite-dimensional representations is useless.

Lemma 6. Let $G$ be a semi-simple connected Lie group with a faithful representation, and let $K$ be a maximal compact subgroup of $G$; then $G$ contains a connected solvable subgroup $N$ such that $G=N \cdot K$ (i.e., every $g \in G$ has a unique representation $g=n \cdot k$ ).

Proof. Since $G$ has a faithful representation, its center is finite (see, for instance, [18]), so that Lemma 6 follows from Lemma 3.12 of Iwasawa's paper [21].

Remark 3. Although a general proof of Lemma 6 requires the use of "deep" theorems concerning Lie algebras, it is quite easy and elementary to verify this lemma for most "classical" groups (unimodular, orthogonal, and symplectic groups); see [12] for the complex case.

The following definition is obviously inspired by [25]:

Definition 5. Let $N$ be a closed subgroup of $G$, and let $n \rightarrow \alpha(n)$ be a onedimensional representation of $N$; let $\mathfrak{S}^{\alpha}$ be the vector space of continuous functions $\theta(g)$ satisfying $\theta(n g)=\alpha(n) \theta(g)$; define a representation $g \rightarrow T_{0}^{\alpha}$ of $G$ on $\mathfrak{S}^{\alpha}$ by 


$$
T_{g}^{\alpha} \theta\left(g^{\prime}\right)=\theta\left(g^{\prime} g\right) ;
$$

then $g \rightarrow T_{0}^{\alpha}$ is called the representation of $G$ induced by $\alpha$; the set of these induced representations is denoted by $\Omega_{N}$.

If $G=N \cdot K$, then $\mathfrak{S}^{\alpha}$ can be identified in a natural way with the Banach space $L(K)$ of continuous functions on $K$ : we have only to associate with every $\theta \in \mathfrak{S}^{\alpha}$ its restriction to $K$.

Lemma 7. Let $N$ be a connected solvable subgroup of $G$; then every finitedimensional irreducible representation of $G$ is contained (as a discrete summand) in some member of $\Omega_{N}$.

Proof. Let $g \rightarrow U_{0}$ be a finite-dimensional irreducible representation of $G$ on $\mathfrak{S}$; consider the contragredient representation $g \rightarrow U_{0}^{\prime}$ of $G$ on the dual space $\mathfrak{S}^{\prime}$; since $N$ is a connected solvable group, the representation $n \rightarrow U_{n}^{\prime}$ of $N$ contains a one-dimensional representation of $N$ (Lie's theorem( $\left.{ }^{(10}\right)$ ); hence there exists a nonvanishing vector $\boldsymbol{a}^{\prime} \in \mathfrak{S g}^{\prime}$ such that we have relations

$$
U_{n}^{\prime} \mathbf{a}^{\prime}=\alpha(n)^{-1} \cdot \mathbf{a}^{\prime}
$$

for every $n \in N$.

Now associate with every $a \in \mathfrak{S}$ the continuous function

$$
\theta_{a}(g)=\left(U_{o} a, a^{\prime}\right) ;
$$

by (12) it is clear that $\theta_{a}(n g)=\alpha(n) \theta_{\mathbf{a}}(g)$, so that $\mathbf{a} \rightarrow \theta_{\mathbf{a}}$ is a linear (and, due to the irreducibility, one-to-one) mapping of $\mathfrak{S}$ onto a finite-dimensional subspace of the space $\mathfrak{S}^{\alpha}$ of the induced representation $g \rightarrow T_{0}^{\alpha}$; since we have obviously

$$
\theta_{U_{0}} \mathbf{a}\left(g^{\prime}\right)=\theta_{\mathbf{a}}\left(g^{\prime} g\right),
$$

Lemma 7 is proved.

THEOREM 2. Let $G$ be a semi-simple connected Lie group with a faithful representation and let $K$ be a maximal compact subgroup of $G$; then every irreducible representation $b$ of $K$ is contained at most $\operatorname{dim}(\mathfrak{b})$ times in every completely irreducible representation of $G$.

Proof. Use the decomposition $G=N \cdot K$ of Lemma 6; by Lemmas 5 and 7 , $\Omega_{N}$ is a complete set of representations of $G$; on the other hand, if we identify every $\mathfrak{S}^{\alpha}$ with $L(K)$, then the representation. $k \rightarrow T_{k}^{\alpha}$ of $K$ reduces to the "regular" representation of $K$ on $L(K)$ (right translations on $L(K)$ ), hence, by well known theorems, contains $\delta$ exactly $\operatorname{dim}(\delta)$ times. Hence Theorem 2 follows from Lemma 4.

Theorem 2 was verified in special cases by Gelfand and Naimark $[10 ; 11$; 12 ], who used also induced representations (but since all representations constructed by Gelfand and Naimark are just induced representations, it was

(10) See a direct proof in the appendix. 
practically trivial to verify the theorem in that case!); general results were announced by Harish-Chandra in [20] (see mainly Theorem 1 in the third Harish-Chandra note), but our method is somewhat simpler.

\section{3 . The case of complex semi-simple groups.}

Theorem 3. Let $G$ be a semi-simple connected complex Lie group and let $K$ be a maximal compact subgroup of $G$; let $\Gamma$ be a maximal abelian subgroup of $K$; for a given $\mathfrak{\delta}$, denote by $n(\mathfrak{b})$ the smallest integer such that every irreducible representation of $\Gamma$ occurs at most $n(\mathfrak{d})$ times in $\mathfrak{d}$; then $\mathfrak{d}$ occurs at most $n(\mathfrak{d})$ times in every completely irreducible representation of $G$.

Proof. Using a modification of Iwasawa's theorem, it is easy to see that we can find a solvable subgroup $T$ of $G$ such that $K \cap T=\Gamma$, and such that every $g \in G$ has at least one (and actually infinitely many!) representation $g=t \cdot k$. Consider then a member $g \rightarrow T_{g}^{\alpha}$ of $\Omega_{T}$; then $\mathfrak{S}^{\alpha}$ can be identified in a natural way with the subspace of $L(K)$ whose elements satisfy $\theta(\gamma k)$ $=\alpha(\gamma) \theta(k)$; using simple arguments due to Gelfand and Naimark $[11 ; 12]$, we conclude that

$$
\text { multiplicity of } \delta \text { in } g \rightarrow T_{0}^{\alpha}=\text { multiplicity of } \gamma \rightarrow \alpha(\gamma) \text { in } \delta \text {; }
$$

hence $\mathfrak{b}$ occurs at most $n(\mathfrak{b})$ times in every member of $\Omega_{T}$; but every complex semi-simple Lie group has a faithful representation (see [18]), so that $\Omega_{T}$ is a complete set (Lemma 7), and Theorem 3 is proved.

Of course, Theorem 3 is inspired by results due to Gelfand and Naimark.

Observe that Theorem 3 leads to a new proof of the corollary of Theorem 1 : we have only to verify that every "weight" of an irreducible representation of $S U(2)$ has multiplicity one.

Theorem 3 implies the following result:

THEOREM 4. Let $K$ be a compact connected semi-simple Lie group, and let $\mathfrak{\delta}, \mathfrak{d}^{\prime}, \mathfrak{d}^{\prime \prime}$ be any three irreducible representations of $K$; let $n(\mathfrak{\delta})$ be the maximal dimension of the various "weight" subspaces of $\mathfrak{b}$; then $\mathfrak{b}$ is contained at most $n(\mathfrak{d})$ times in $\mathfrak{d}^{\prime} \otimes \mathfrak{d}^{\prime \prime}$.

Proof. Imbed $K$ in the associated "algebraic group" $G$ (see Chevalley [3]), observe that $\mathfrak{d}^{\prime} \otimes \mathfrak{d}^{\prime \prime}$ is the restriction of some finite-dimensional irreducible representation of $G$, and apply Theorem 3 to $G$.

REMARK 4 . Let $K$ be an arbitrary compact group, and let $\delta$, $\mathfrak{\delta}^{\prime}$, and $\mathfrak{\delta}^{\prime \prime}$ be any three irreducible representations of $K$; denote by $\chi_{0}, \chi_{0^{\prime}}$ and $\chi_{\delta^{\prime \prime}}$ their characters in the usual normalization (so that $\chi_{b}(e)=\operatorname{dim}(\delta)$ ). Then the number of times $\mathfrak{d}$ occurs in $\mathfrak{d}^{\prime} \otimes \mathfrak{d}^{\prime \prime}$ is given by

$$
N=\int \bar{\chi}_{\mathfrak{b}}(k) \chi_{\boldsymbol{b}^{\prime}}(k) \chi_{\boldsymbol{b}^{\prime \prime}}(k) d k ;
$$

since $\left|\chi_{\mathfrak{b}}(k)\right| \leqq \chi_{\mathfrak{b}}(e)=\operatorname{dim}(\mathfrak{d})$, we get 


$$
N \leqq \operatorname{dim}(\delta) \int\left|\chi_{\mathfrak{D}^{\prime}}(k) \chi_{\mathfrak{b}^{\prime \prime}}(k)\right| d k
$$

but (orthogonality relations) we have

$$
\int\left|\chi_{\mho^{\prime}}(k)\right|^{2} d k=\int\left|\chi_{\jmath^{\prime \prime}}(k)\right|^{2} d k=1 ;
$$

hence $N \leqq \operatorname{dim}(\mathfrak{d})$, and we therefore obtain:

REFEREE'S THEOREM. Let $\mathfrak{\delta}, \mathfrak{b}^{\prime}, \mathfrak{d}^{\prime \prime}$ be any three irreducible representations of an arbitrary compact group $K$; then $\delta$ occurs at most $\operatorname{dim}(\delta)$ times in $\delta^{\prime} \otimes \delta^{\prime \prime}$.

\section{Motion groups.}

TheOREM 5. Let $G$ be a locally compact group, $K$ a compact subgroup of $G$; assume that $G=N \cdot K$ where $N$ is an abelian subgroup of $G$; then every irreducible representation of $K$ occurs at most dim (ס) times in every completely irreducible representation of $G$.

Proof. As in the proof of Theorem 2, we consider the set $\Omega_{N}$ of representations of $G$ induced by one-dimensional representations of $N$; clearly, Theorem 5 will be proved if we establish that $\Omega_{N}$ is a complete set. Of course, we cannot use Lemma 7 , so that we have to give a direct proof.

Consider a given $f \in L(G)$, and assume that $T_{f}^{\alpha}=0$ for every one-dimensional representation $n \rightarrow \alpha(n)$ of $N$; we must prove that $f=0$. In order to make the situation clear, we shall not use now the assumption that $N$ is abelian; we shall use only the fact that $G=N \cdot K$.

Denote by $d n$ the left-invariant Haar measure on $N$; by the theory of homogeneous spaces we may assume that

$$
\int f(g) d g=\iint f(n k) d n d k
$$

for every $f \in L(G)$. Now if $f \in L(G)$ and $\theta \in \mathfrak{S}^{\alpha}$, we have

$$
\begin{aligned}
T_{f}^{\alpha} \theta(k) & =\int T_{0}^{\alpha} \theta(k) \cdot f(g) d g=\int \theta(k g) f(g) d g=\int \theta(g) f\left(k^{-1} g\right) d g \\
& =\iint \theta\left(n k^{\prime}\right) f\left(k^{\prime \prime} n k^{\prime}\right) d n d k^{\prime}=\iint \theta\left(k^{\prime}\right) \alpha(n) f\left(k^{\prime \prime} n k^{\prime}\right) d n d k^{\prime}
\end{aligned}
$$

where we set $k^{\prime \prime}=k^{-1}$ and where we use the definition of $\mathfrak{S}^{\alpha}: \theta(n g)=\alpha(n) \theta(g)$. If $T_{f}^{\alpha}=0$, we have therefore

$$
\int f\left(k^{\prime \prime} n k^{\prime}\right) \alpha(n) d n=0
$$


for any $k^{\prime}, k^{\prime \prime} \in K$, since we must have $T_{f}^{\alpha} \theta=0$ for every $\theta \in L(K)$. Now denote by $Z$ the subgroup of $N$ defined by the condition that $\alpha(z)=1$ for every onedimensional representation $\alpha$ of $N$; of course, $Z$ is the topological commutator group of $N$, and $N / Z$ is an abelian group; denote by $n \rightarrow \dot{n}$ the natural mapping of $N$ onto $N / Z$. Since every one-dimensional representation of $N$ reduces on $Z$ to the identity, it is clear that: (1) $d n$ is invariant under $n \rightarrow n z$; (2) $Z$ is a unimodular group; hence we have an integration formula

$$
\int f(n) d n=\int d \dot{n} \int f(n z) d z,
$$

so that (16) implies

$$
\int \alpha(\dot{n}) d \dot{n} \int f\left(k^{\prime} n z k^{\prime \prime}\right) d z=0 ;
$$

but since $\dot{n} \rightarrow \alpha(\dot{n})$ is an arbitrary character of the abelian group $N / Z$, this means that

$$
\int f\left(k^{\prime} n z k^{\prime \prime}\right) d z=0
$$

Now we use the assumption that $N$ is abelian; then $Z=e$, so that (18) reduces to $f\left(k^{\prime} n k^{\prime \prime}\right)=0$, hence means that $f=0$, and Theorem 5 is proved.

REMARK 5. If $G=S L(n, C)$ and if we use (as in the proof of Theorem 3) the subgroup $T$ with $K \cap T=\Gamma$, then it follows from Plancherel's formula on $G$ that the set of representations in $\Omega_{T}$ which are unitary [of course, with respect to the scalar product $\left(f^{\prime}, f^{\prime \prime}\right)=\int f^{\prime}(k) \bar{f}^{\prime \prime}(k) d k$ on $\left.L(G)\right]$ is complete. We can now prove this in a direct way; in fact, $g \rightarrow T_{0}^{\alpha}$ is unitary if and only if

$$
\int\left|T_{o}^{\alpha} \theta(k)\right|^{2} d k=\int|\theta(k)|^{2} d k
$$

for every $\theta \in L(K)$ satisfying $\theta(\gamma k)=\alpha(\gamma) \theta(k)$; but the above relation means $\int|\theta(k g)|^{2} d k=\int|\theta(k)|^{2} d k$ where $\theta$ is extended to $G$ by the requirement that $\theta(t k)=\alpha(t) \theta(k)$, hence is equivalent to the fact that

$$
\iint|\theta(k g)|^{2} f(g) d k d g=\iint|\theta(k)|^{2} f(g) d k d g
$$

for every $f \in L(G)$; but we may use the decomposition $G=N \cdot K$ of Lemma 6 and assume that $T=N \cdot \Gamma$; (19) is then equivalent to

$$
\iiint\left|\theta\left(n k^{\prime}\right)\right|^{2} f\left(k^{-1} n k^{\prime}\right) d k d k^{\prime} d n=\iint\left|\theta\left(k^{\prime}\right)\right|^{2} f(g) d k^{\prime} d g,
$$


i.e., by $\theta\left(n k^{\prime}\right)=\alpha(n) \theta\left(k^{\prime}\right)$, and since $\theta$ is more or less arbitrary in $L(K)$, to

$$
\iint f\left(k n k^{\prime}\right)|\alpha(n)|^{2} d k d n=\int f(g) d g
$$

but from the integration formula $\int f(g) d g=\iint f(n k) d n d k$ it is easy to deduce that $\int f(g) d g=\iint f(k n) \beta(n) d k d n$, where we set $d\left(n^{-1}\right)=\beta(n) d n$; hence (21) implies $\left(k^{\prime}=e\right)$

$$
\iint f(k n)|\alpha(n)|^{2} d k d n=\iint f(k n) \beta(n) d k d n
$$

and finally we see that $g \rightarrow T_{g}^{\alpha}$ is unitary if and only if

$$
|\alpha(n)|=\beta^{1 / 2}(n), \text { where } d\left(n^{-1}\right)=\beta(n) d n .
$$

Of course, this is not new; see Gelfand and Naimark [8;12].

Now to prove that the corresponding induced representations form a complete set we use the same arguments as in the proof of Theorem 5, and instead of (17) for every $\alpha$ we get (17) for every $\alpha$ satisfying (23); but this already implies (18), because the abelian group $N / Z$ has a complete set of bounded characters; hence if $f \in L(G)$ satisfies $T_{f}^{\alpha}=0$ whenever $g \rightarrow T_{a}^{\alpha}$ is unitary, then $f$ satisfies (18), hence satisfies $T_{f}^{\alpha}=0$ for arbitrary $\alpha$, so that $f=0$.

REMARK 6. Of course, Theorem 5 is useful mainly in connection with "motion groups," i.e., in the case where the abelian subgroup $N$ is invariant. The study of such groups is much easier than that of semi-simple groups, but it is important to study both classes in order to get a theory of "spherical functions" including Bessel functions as a special case.

\section{MAXIMAL IDEALS IN GROUP ALGEBRAS}

We recall that a left ideal $\mathfrak{m}$ in an associative algebra $\boldsymbol{A}$ is said to be regular if there exists some $u \in A$ such that $x u \equiv x(\bmod \mathfrak{m})$ for every $x \in A$; similar definitions apply to right ideals and to two-sided ideals. We also recall that every regular left ideal is contained in at least one maximal left ideal, which is regular; in a complete normed algebra every regular maximal ideal is closed.

Let $G$ be a locally compact group, $\rho(g)$ a semi-norm on $G, A_{\rho}(G)$ the complete normed algebra of absolutely integrable functions with respect to the measure $\rho(g) d g$, and consider a regular maximal left ideal $\mathrm{m}$ in $\boldsymbol{A}_{\rho}(G)$; since $\mathfrak{m}$ is closed it is invariant under left translations, so that we can define in a natural way a representation $g \rightarrow T_{o}$ of $G$ on the Banach space $A_{\rho}(G) / \mathrm{m}$; the corresponding representation of $A_{\rho}(G)$ is obtained by associating with $f \in A_{\rho}(G)$ the operator which transforms the class (modulo $\mathfrak{m}$ ) of $\theta \in A_{\rho}(G)$ into the class of $f \theta$ (convolution product); hence this representation of $\boldsymbol{A}_{\rho}(G)$ is algebraically irreducible, so that the representation $g \rightarrow T_{0}$ of $G$ is completely irreducible by Lemma 2 ; in addition, if we denote by $u$ a right identity 
modulo $\mathfrak{m}$ and by $\dot{u}$ the class of $u$ in $A_{\rho}(G) / \mathfrak{m}$, then it is clear that $f \in \mathfrak{m}$ is equivalent to $T_{f} \dot{u}=0$.

Take conversely a completely irreducible representation $g \rightarrow T_{0}$ of $G$ on a Banach space $\mathfrak{S}$, and for a given vector $\boldsymbol{u} \in \mathfrak{S}$ denote by $\mathfrak{m}$ the set of $f \in A_{\rho}(G)$ such that $T_{f} u=0$ (of course, we choose $\rho(g)=\left\|T_{o}\right\|$ ); it is obvious that $\mathfrak{m}$ is a closed left ideal in $\boldsymbol{A}_{\rho}(G)$; but it is not known whether $\mathrm{m}$ is a regular maximal left ideal in $\boldsymbol{A}_{\rho}(G)$, except of course for abelian and for compact groups, i.e., for groups whose completely irreducible representations are finite-dimensional; and actually there are reasons to believe that, in "general" groups, this conjecture is false. Our purpose in this section is to prove that, from this point of view, semi-simple Lie groups and motion groups behave in a relatively normal way. We shall, namely, prove the following theorem:

THEOREM 6. Let $g \rightarrow T_{o}$ be a topologically irreducible representation of a locally compact group $G$ on a Banach space $\mathfrak{S}$; for a given irreducible representation $\mathfrak{d}$ of a given compact subgroup $K$ of $G$, assume that $\operatorname{dim} \mathfrak{S}(\mathfrak{D})<+\infty$; then for every nonvanishing vector $u \in \mathfrak{S}(\mathfrak{b})$ the set $\mathfrak{m}$ of $f \in A_{\rho}(G)$ such that $T_{f} \mathbf{u}=0$ is a regular maximal left ideal in $\boldsymbol{A}_{\rho}(G)$.

5. A general lemma. To simplify the terminology, we shall say that an associative algebra $\boldsymbol{A}$ over the complex field is a group algebra of $G$ if $\boldsymbol{A}$ is a two-sided module over the ring $\boldsymbol{M}(G)$ of measures with compact carrier on $G$; hence for every $f \in \boldsymbol{A}$ and every $\alpha \in \boldsymbol{M}(G)$ we may define $\alpha f$ and $f \alpha$, and all usual associative rules are satisfied.

In such a group algebra $A$, we can of course define subalgebras $A(\mathfrak{D})$ by the condition

$$
\bar{\chi}_{\triangleright} f=f \bar{\chi}_{b}=f .
$$

Lemma 8. Let $\boldsymbol{A}$ be a group algebra of $G$ and let $\mathfrak{a}$ be a regular maximal left ideal in $A(\mathfrak{d})$; let $\mathfrak{m}$ be the set of $f \in A$ such that

$$
\bar{\chi}_{\triangleright} g f \bar{\chi}_{b} \in \mathfrak{a} \quad \text { for every } g \in A \text {; }
$$

then $\mathfrak{m}$ is a regular maximal left ideal in $\boldsymbol{A}, \mathfrak{a}=\mathfrak{m} \cap \boldsymbol{A}(\mathfrak{b})$, and we have f $\bar{\chi}_{\mathfrak{b}}$ $\equiv f(\bmod \mathfrak{m})$ for every $f \in A$.

Proof. The fact that $\mathfrak{m}$ is a left ideal is obvious; to see that $\mathfrak{m}$ is regular, take a right identity $u \in A(\mathfrak{d})$ modulo $\mathfrak{a}$; for arbitrary $f, g \in A$ we have $\bar{\chi}_{\triangleright} g(f u-f) \bar{\chi}_{\delta}=h u-h$ where $h=\bar{\chi}_{\triangleright} g f \bar{\chi}_{b} \in A(\delta)$; hence $u$ is a right identity modulo $\mathfrak{m}$. To prove that $\mathfrak{a}=\mathfrak{m} \cap \boldsymbol{A}(\mathfrak{b})$ it is enough (by the maximality of $\mathfrak{a}$ ) to prove that $\mathfrak{m} \cap A(\mathfrak{b})$ is a nontrivial ideal containing $\mathfrak{a}$; but $u \in \mathfrak{m}$ would imply $\bar{\chi}_{\triangleright} f u \bar{\chi}_{\triangleright} \in \mathfrak{a}$ for every $f \in A$, hence would imply $f u \in \mathfrak{a}$ for every $f \in A(\delta)$, hence would imply that $\mathfrak{a}=\boldsymbol{A}(\mathfrak{b})$; hence $\mathfrak{m}$ does not contain $u$, so that it remains to prove that $\mathfrak{a} \subset \mathfrak{m}$; but take any $f \in \mathfrak{a}$; for every $g \in \boldsymbol{A}$ we have 
$\bar{\chi}_{\triangleright} g f \bar{\chi}_{b}=\bar{\chi}_{\triangleright} g \bar{\chi}_{b} f \in \mathfrak{a}$ since $\bar{\chi} \diamond s \bar{\chi}_{\mathfrak{b}} \in A(\mathfrak{d})$, and this concludes the proof $\left({ }^{11}\right)$ of $\mathfrak{a}=\mathfrak{m}$ $\cap \boldsymbol{A}(\delta)$. The fact that $f \bar{\chi}_{b}-f \in \mathfrak{m}$ for every $f$ follows from $\bar{\chi}_{\delta} g\left(f \bar{\chi}_{b}-f\right) \bar{\chi}_{b}$ $=\bar{\chi}_{\diamond} g f \bar{\chi}_{\diamond} \bar{\chi}_{b}-\bar{\chi}_{\diamond} g f \bar{\chi}_{b}=0$. Now we can prove that $\mathfrak{m}$ is maximal. Let $\mathfrak{n}$ be a left ideal containing $\mathfrak{m}$ and assume $\mathfrak{n} \neq \boldsymbol{A}$, so that $u \notin \mathfrak{n}$; by the maximality of $\mathfrak{a}$ we see that $\mathfrak{n} \cap \boldsymbol{A}(\mathfrak{d})=\mathfrak{a}$; but if $f \in \mathfrak{n}$, we have $f \bar{\chi}_{\mathfrak{b}}-f \in \mathfrak{m} C \mathfrak{n}$, hence $f \bar{\chi}_{\mathfrak{b}} \in \mathfrak{n}$, hence (since $\mathfrak{n}$ is a left ideal) $\bar{\chi}_{\mathfrak{b}} g f \bar{\chi}_{\mathfrak{b}} \in \mathfrak{n}$ for every $g$, hence $\bar{\chi}_{\triangleright} g f \bar{\chi}_{\mathfrak{b}} \in \mathfrak{n} \cap \boldsymbol{A}(\mathfrak{b})=\mathfrak{a}$; hence $f \in \mathfrak{n}$ implies $f \in \mathfrak{m}$, and Lemma 7 is entirely proved.

6. Proof of Theorem 6. With the notation of Theorem 6, consider the left ideal $\mathfrak{a}=\mathfrak{m} \cap A_{\rho}(\mathfrak{d}) \quad\left(A_{\rho}(\mathfrak{d})\right.$ is the set of $f \in A_{\rho}(G)$ satisfying $\bar{\chi}_{\mathfrak{b}} f=f \bar{\chi}_{\mathfrak{b}}=f$; of course, $\boldsymbol{A}_{\rho}(G)$ is a group algebra!) and consider the representation $f \rightarrow \widetilde{T}_{f}$ of $A_{\rho}(\mathfrak{d})$ on $\mathfrak{S}(\mathfrak{b})$, defined as in no. $1 ; \mathfrak{a}$ is given by $\widetilde{T}_{f} \mathfrak{u}=0$; but since $g \rightarrow T_{g}$ is topologically irreducible, the same is true for the representation $f \rightarrow \widetilde{T}_{f}$ of $\boldsymbol{A}_{\boldsymbol{\rho}}(\mathfrak{D})$ (Lemma 3 suitably modified $\left({ }^{12}\right)$ ), and since we assume $\operatorname{dim} \mathfrak{S}(\mathfrak{D})<+\infty$ we see that $f \rightarrow \widetilde{T}_{f}$ is algebraically irreducible; hence $\mathfrak{a}$ is a regular maximal left ideal in $\boldsymbol{A}_{\boldsymbol{\rho}}(\boldsymbol{\delta})$.

Now consider the regular maximal left ideal $\mathfrak{m}^{\prime}$ in $A_{\rho}(G)$ defined by applying to $\mathfrak{a}$ the process of Lemma $8 ; f \in \mathfrak{m}^{\prime}$ means that $\bar{\chi}_{\triangleright} g f \bar{\chi}_{\mathfrak{b}} \in \mathfrak{a}$ for every $g$; but it is clear that, if we set $h=\bar{\chi}_{\triangleright} g f \bar{\chi}_{\triangleright}$, then $\widetilde{T}_{h} \boldsymbol{u}=T_{h} \boldsymbol{u}=E(\mathfrak{\delta}) T_{\mathfrak{o}} T_{f} E(\mathfrak{b}) u$ $=E(\mathfrak{b}) T_{o} T_{f} u$; hence $f \in \mathfrak{m}^{\prime}$ means that the invariant subspace of $\mathfrak{S}$ generated by $T_{f} u$ is "orthogonal" to $\mathfrak{S}(\delta)$; but since the given representation of $G$ is topologically irreducible this invariant subspace either vanishes (and then $f \in \mathfrak{m}$ ) or is everywhere dense in $\mathfrak{S}$ (which cannot arise, because this would imply $\mathfrak{S}(\mathfrak{b})=0$ ); hence $\mathfrak{m}=\mathfrak{m}^{\prime}$, and Theorem 6 follows from Lemma 8 .

Remark 7. Of course Theorem 6 applies also to $L(G)$ instead of $A_{\rho}(G)$, and also to $\boldsymbol{M}(G)$-more generally, Theorem 6 applies to every group algebra $\boldsymbol{A}$ provided $T_{f}$ can be defined in a reasonable way for every $f \in \boldsymbol{A}$.

7. Consequences of Theorem 6 . Let $g \rightarrow T_{g}$ be a topologically irreducible representation of $G$ on a Banach space $\mathfrak{S}$, and assume that $\mathfrak{S}(\mathfrak{d})$ is finitedimensional for every $\mathfrak{b}$. Set $\rho(g)=\left\|T_{\vartheta}\right\|$, and for a given $\boldsymbol{a} \in \mathfrak{S}(\mathfrak{b})$ let $\mathfrak{S}_{0}$ be the set of vectors $T_{f} a, f \in A_{\rho}(G)$. Of course, $\mathfrak{S}_{0}$ is invariant under the given representation of $G$, and the representation $f \rightarrow T_{f}$ of $A_{\rho}(G)$ on $\mathfrak{S}_{0}$ is algebraically irreducible.

(11) The fact that $\mathfrak{a}=\mathfrak{m} \cap \boldsymbol{A}(\boldsymbol{b})$ can be proved in many cases without assuming that $\mathfrak{a}$ is maximal. In fact if $f \in \boldsymbol{A}(\boldsymbol{\delta})$, then we have $\bar{\chi}_{\delta} g f \bar{\chi}_{\delta}=\bar{\chi}_{\delta} g \bar{\chi}_{\triangleright} f$ for every $g \in \boldsymbol{A}$, so that the ideal $\mathfrak{a}^{\prime}=\mathfrak{m} \cap \boldsymbol{A}(\mathfrak{b})$ is the set of $f \in A(\mathfrak{b})$ such that $g f \in \mathfrak{a}$ for every $g \in A(\mathfrak{b})$; if, for instance, $\mathfrak{a}$ is not only a left ideal but a regular two-sided ideal in $A(b)$ and if $u \in A(b)$ is a unit modulo a, then the above property implies $u f \in \mathfrak{a}$ and since $u f-f \in \mathfrak{a}$, we see that in this case $\mathfrak{a}^{\prime}=\mathfrak{a}$; on the other hand assume that $\boldsymbol{A}=\boldsymbol{A}_{\rho}(G)$ for some semi-norm $\rho$ and assume that the regular left ideal $a$ is closed in $A_{\rho}(\delta)$; by the well known "smoothing method" it can be proved that, for every $f \in A_{\rho}(G), f$ is a strong limit of functions $g f, g \in A_{\rho}(G)$; if $f \in A_{\rho}(\mathcal{\delta})$, we may of course assume that $g \in A_{\rho}(\delta)$; hence if $f \in \mathfrak{a}^{\prime}$, then $f$ is a strong limit of functions belonging to $\mathfrak{a}$, hence belongs to $\mathfrak{a}$ since $\mathfrak{a}$ is closed, so that relation $\mathfrak{a}=\mathfrak{m} \cap \boldsymbol{A}_{\rho}(\mathfrak{b})$ still holds in this case.

(12) Proof. Let $a \in \mathfrak{S}(\delta)$; by the topological irreducibility of $f \rightarrow T_{f}$ we can approximate a by vectors $T_{f} u, f \in A_{p}(G)$; since $E(\delta) a=a$, we can therefore approximate a by vectors $E(\delta) T_{f} u$,

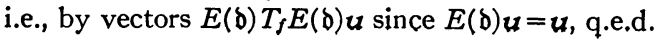


The subspace $\mathfrak{S}_{0}$ depends neither on $a \in \mathfrak{S}(\mathfrak{D})$ nor on $\mathfrak{b}$. In fact for every $\mathfrak{d}^{\prime}, E\left(\mathfrak{d}^{\prime}\right) \mathfrak{S}_{0}$ is everywhere dense in $\mathfrak{S}\left(\mathfrak{d}^{\prime}\right)$ since $\mathfrak{S}_{0}$ is everywhere dense in $\mathfrak{S}$; since $\mathfrak{S}\left(\mathfrak{b}^{\prime}\right)$ is finite-dimensional, this implies $E\left(\mathfrak{b}^{\prime}\right) \mathfrak{S}_{0}=\mathfrak{S}\left(\mathfrak{b}^{\prime}\right)$, and since $\mathfrak{S}_{0}$ is obviously invariant under every $E\left(\mathfrak{d}^{\prime}\right)$ we see that $\mathfrak{S}\left(\mathfrak{d}^{\prime}\right) \subset \mathfrak{S}_{0}$ for every $\mathfrak{b}^{\prime}$; hence if instead of $\mathfrak{b}$ and $a \in \mathfrak{S}(\mathfrak{b})$ we consider $\mathfrak{b}^{\prime}$ and $\boldsymbol{a}^{\prime} \in \mathfrak{S}\left(\mathfrak{b}^{\prime}\right)$, the new subspace $\mathfrak{S}_{0}^{\prime}$ will be contained in $\mathfrak{S}_{0}$, hence will be identical with $\mathfrak{S}_{0}$ by the algebraical irreducibility of $f \rightarrow T_{f}$ on $\mathfrak{S}_{0}$.

We thus see that there exists a natural way of restricting the given representation to a representation on an everywhere dense subspace $\mathfrak{S}_{0}$, in order to get an algebraically irreducible representation of $G$ (i.e., of $\boldsymbol{A}_{\rho}(G)$ ). Since $\mathfrak{S}_{0}$ is isomorphic with the factor space of $\boldsymbol{A}_{\rho}(G)$ modulo a closed ideal, we can consider $\mathfrak{S}_{0}$ as a Banach space; in a precise way, to get a norm on $\mathfrak{S}_{0}$ such that $\mathfrak{S}_{0}$ becomes a complete normed vector space we choose a nonvanishing vector a belonging to some $\mathfrak{E}(\mathfrak{b})$, and for every $\mathbf{x} \in \mathfrak{S}_{0}$ we define $\|\boldsymbol{x}\| \mid$ $=\inf \|f\|_{\rho}$, where the inf runs over the set of functions $f \in A_{\rho}(G)$ such that $\mathbf{x}=T_{f} \mathbf{a}$. Of course, the new norm depends on $\mathbf{a}$; but by a classical theorem due to Banach or by direct computation it is obvious that the norms defined by two vectors $\mathbf{a} \in \mathfrak{S}(\mathfrak{D})$ and $\mathbf{a}^{\prime} \in \mathfrak{S}\left(\mathfrak{b}^{\prime}\right)$ are equivalent.

The above results show that, on a semi-simple Lie group with a faithful representation, every completely irreducible representation contains a welldefined representation which, extended to the corresponding algebra $\boldsymbol{A}_{\rho}(G)$, is algebraically irreducible.

Concerning the structure of the operators $T_{f}$, it is important to note the following result:

TheOREM 7. Assume that $\operatorname{dim} \mathfrak{S}(\mathfrak{D})<+\infty$ for every $\mathfrak{d}$; then every operator $T_{f}, f \in A_{\rho}(G)$, is completely continuous.

Proof. If $\bar{\chi}_{b} f=f$, we have $E(\mathfrak{D}) T_{f}=T_{f}$, and since $E(\mathfrak{d})$ has a finite rank the theorem is obvious in that case. Now take an arbitrary $f \in A_{\rho}(G)$; if we denote by $L_{k}$ the left translation $f(g) \rightarrow f\left(k^{-1} g\right)$ on $A_{\rho}(G)$ and if we decompose that representation of $K$ it is obvious that the corresponding projection operators $E(\delta)$ are given by $f \rightarrow \chi \diamond f$; using Péter-Weyl's theorem we thus see that $f$ is a strong limit of linear combinations of functions $\chi_{b} f$; since we have $\left\|T_{f}\right\| \leqq\|f\|_{\rho}$, this proves that $T_{f}$ is a uniform limit of operators of finite rank, q.e.d.

For representations on Hilbert spaces, more precise results were announced by Harish-Chandra (see third note [20]).

8. The kernel of a representation. Let $g \rightarrow T_{0}$ be a topologically irreducible representation, and assume that every $\mathfrak{W}(\mathfrak{D})$ is finite-dimensional. The set of functions $f \in A_{\rho}(G)$ such that $T_{f}=0$ is of course a closed two-sided ideal; one might think that it is a maximal two-sided ideal-but this is not true; for if this were true, then the algebra of operators $T_{f}$ would be simple, and, since it contains nonvanishing operators of finite rank (namely the $T_{f}$ where 
$\chi_{\triangleright} f=f$ for some $\delta$ ), this would prove that every $T_{f}$ is of finite rank, which is obviously false.

However, one can prove that the ideal $\mathfrak{a}$ defined by $T_{f}=0$ is maximal in the set of closed two-sided ideals in $A_{\rho}(G)$. In fact let $\mathfrak{b}$ be a closed two-sided ideal containing $\mathfrak{a}$, and assume that $\mathfrak{b} \neq A_{\rho}(G)$. For every $\mathfrak{b}$ we have $\mathfrak{a} \cap A_{\rho}(\mathfrak{d})$ $\subset \mathfrak{b} \cap \boldsymbol{A}_{\rho}(\mathfrak{D})$; but $\mathfrak{a} \cap \boldsymbol{A}_{\rho}(\mathfrak{b})$ is the kernel of the finite-dimensional irreducible representation $f \rightarrow \widetilde{T}_{f}$ of $A_{\rho}(\mathfrak{D})$ on $\mathfrak{S}(\mathfrak{D})$, hence is a regular maximal two-sided ideal in $A_{\rho}(\mathfrak{d})$ so that we have either $\mathfrak{a} \cap A_{\rho}(\mathfrak{d})=\mathfrak{b} \cap A_{\rho}(\mathfrak{d})$ or $\mathfrak{b} \cap A_{\rho}(\mathfrak{d})=A_{\rho}(\mathfrak{d})$. Now assume that $\mathfrak{b}$ contains every $\boldsymbol{A}_{\rho}(\mathfrak{d})$; then we have $f \chi_{\triangleright} g \chi_{\triangleright} h \in \mathfrak{b}$ for arbitrary $f, g, h$ and arbitrary $\mathfrak{b}$; but if we assume that the measure $g(x) d x$ converges to $\epsilon$, then $f \chi_{\diamond} g \chi_{\diamond} h$ converges to $f \chi_{\triangleright} h$, and since $\mathfrak{b}$ is closed, we see that $f \chi_{\triangleright} h \in \mathfrak{b}$; if now $h(x) d x$ converges to $\epsilon$, then by the same argument we get $f \chi_{\mathfrak{b}} \in \mathfrak{b}$ for arbitrary $f \in A_{\rho}(G)$ and arbitrary $\mathfrak{b}$; but by Péter-Weyl's theorem $f$ can be approximated in $\boldsymbol{A}_{\rho}(G)$ by linear combinations of functions $f \chi_{\triangleright}$, and we finally see that our assumption implies $\mathfrak{b}=A_{\rho}(G)$.

If $\mathfrak{b} \neq A_{\rho}(G)$, we have therefore $\mathfrak{a} \cap A_{\rho}(\mathfrak{b})=\mathfrak{b} \cap A_{\rho}(\mathfrak{b}) \neq A_{\rho}(\mathfrak{d})$ for at least one $\mathfrak{b}$. Now take an arbitrary $f \in \mathfrak{b}$; since $\mathfrak{b}$ is a two-sided ideal we have

$$
\bar{\chi}_{\mathfrak{b}} g f h \bar{\chi}_{\mathfrak{b}} \in \mathfrak{b} \cap A_{\rho}(\mathfrak{(})=\mathfrak{a} \cap A_{\rho}(\mathfrak{d})
$$

for arbitrary $g, h \in A_{\rho}(G)$, which means that

$$
E(\mathfrak{d}) T_{g} T_{f} T_{h} E(\mathfrak{d})=0
$$

for arbitrary $g, h \in A_{\rho}(G)$; taking $a \in \mathfrak{S}(\delta)$ and setting $b=T_{f} T_{h} a$ we see that $E(\delta) T_{b} b=0$ for every $g$, so that by the topological irreducibility of the given representation and the fact that $E(\mathfrak{d}) \neq 0$ [which follows from $a \cap A_{\rho}(\mathfrak{d})$ $\left.\neq A_{\rho}(b)\right]$ we see that $b=0$; hence $T_{f}$ maps all vectors $T_{h} a, h \in A_{\rho}(G)$, a $\in \mathfrak{S}(\mathfrak{b})$, on 0 , which proves that $T_{\mathfrak{f}}=0$. Hence $f \in \mathfrak{b}$ implies $f \in \mathfrak{a}$, and this concludes the proof of our assertion.

\section{FunCTIONAL PROPERTIES OF SPHERICAL FUNCTIONS}

Throughout this section, we assume that $G$ is a locally compact group with a compact subgroup $K$ such that, for every $\delta$, every completely irreducible representation of $L(\delta)$ is finite-dimensional. Hence, for every completely irreducible representation $g \rightarrow T_{g}$ of $G$ on a Banach space $\mathfrak{S}$, the subspaces $\mathfrak{S}(\mathfrak{D})$ are finitedimensional, so that we may consider the functions

$$
\phi_{\mathrm{b}}(g)=\operatorname{Tr}\left[E(\mathfrak{b}) T_{g}\right]
$$

where $\operatorname{Tr}$ is the usual trace, defined at least for operators of finite rank. The function (25) will be called the spherical function of type $b$ of the given representation, and if $b$ occurs $p$ times in that representation, then we shall say that $\phi_{b}$ has height $p$. Our purpose is to study the connections between spherical functions and representations of subalgebras of $L(G)$, in order to generalize the well known theorem to the effect that, if $G$ is an abelian group, then 
every one-dimensional representation of $L(G)$ is given by $f \rightarrow \int f(g) \chi(g) d g$ where $\chi$ is a character of $G$. Observe that abelian groups are included in the class of groups we are going to study: take $G$ abelian and $K$ reduced to the identity. Also our theory will include that of characters of compact groups: take $G$ compact and $K$ reduced to the identity (one could also take $G=K$ ).

9. Elementary properties of spherical functions. A first obvious property of spherical functions is their invariance under $K$, i.e.,

$$
\phi_{\mathrm{D}}\left(k g k^{-1}\right)=\phi_{\mathrm{D}}(g) \text {; }
$$

the proof of this can be left to the reader. Another property is

$$
\chi_{\triangleright} \phi_{b}=\phi_{\triangleright} \chi_{b}=\phi_{b} ;
$$

in fact take a measure $\alpha \in M(G)$ and denote in a general way by $\alpha^{\prime}$ the transform of $\alpha$ under $g \rightarrow g^{-1}$; then we have

$$
\alpha^{\prime} \phi_{\mathrm{b}}(g)=\int \phi_{\mathrm{b}}\left(g^{\prime} g\right) d \alpha\left(g^{\prime}\right)=\int \operatorname{Tr}\left[E(\mathfrak{b}) T_{g^{\prime}} T_{g}\right] d \alpha\left(g^{\prime}\right),
$$

i.e.,

$$
\alpha^{\prime} \phi_{\mathrm{b}}(g)=\operatorname{Tr}\left[E(\mathrm{\delta}) T_{\alpha} T_{g}\right] ;
$$

taking $\alpha=\bar{\chi}_{b}$ and observing that $\alpha^{\prime}=\chi_{b}$ we get the first half of (27); the second half follows from (26) and the first.

A third important property is

$$
\int \phi_{\mathrm{D}}\left(g k g^{\prime}\right) \bar{\chi}_{\mathrm{D}}(k) d k=\int \phi_{\mathrm{D}}\left(g^{\prime} k g\right) \bar{\chi}_{\mathrm{D}}(k) d k ;
$$

in fact we have

$$
\begin{aligned}
& \int \phi_{\mathrm{D}}\left(g k g^{\prime}\right) \bar{\chi}_{\mathrm{D}}(k) d k=\int \operatorname{Tr}\left[E(\mathfrak{D}) T_{g} T_{k} T_{g^{\prime}}\right] \bar{\chi}_{\mathfrak{D}}(k) d k \\
& =\operatorname{Tr}\left[E(\mathfrak{\delta}) T_{g} E(\mathfrak{d}) T_{g^{\prime}}\right]
\end{aligned}
$$

and since the last expression is obviously symmetric with respect to $g$ and $g^{\prime}$ we get (29).

Another property similar to (29) is that

$$
\int \phi_{\mathrm{D}}\left(k g k^{-1} g^{\prime}\right) d k=\int \phi_{\mathrm{D}}\left(k g^{\prime} k^{-1} g\right) d k
$$

holds for every $g, g^{\prime} \in G$. In fact, if we set $T_{o}^{\circ}=\int T_{k} T_{o} T_{\mathbf{k}}^{-1} \cdot d k$, then it is clear that the left-hand side of $(31)$ is identical to $\operatorname{Tr}\left[E(\mathfrak{b}) T_{0}^{\circ} T_{g^{\prime}}\right]$; but it is obvious 
that $E(\mathfrak{b})$ and $T_{0}^{\circ}$ commute with every $T_{k}$ so that we can write

$$
\begin{aligned}
& \operatorname{Tr}\left[E(\mathfrak{d}) T_{g}^{\circ} T_{g^{\prime}}\right\rfloor=\operatorname{Tr}\left[T_{k} E(\mathfrak{\delta}) T_{g}^{\circ} T_{g^{\prime}} T_{k}^{-1}\right]=\operatorname{Tr}\left[E(\mathfrak{b}) T_{g}^{\circ} T_{k} T_{g^{\prime}} T_{k}^{-1}\right] \\
& =\int \operatorname{Tr}\left[E(\mathfrak{D}) T_{g}^{\circ} T_{k} T_{g^{\prime}} T_{k}^{-1}\right] d k=\operatorname{Tr}\left[E(\delta) T_{o}^{\circ} T_{g^{\prime}}^{\circ}\right] \text {, }
\end{aligned}
$$

and since every $T_{g}^{\circ}$ commutes with $E(\delta)$, we see that the left-hand side of (31) is symmetric with respect to $g$ and $g^{\prime}$.

It is important to observe that (31) is equivalent to the fact that

$$
f \phi_{\mathrm{b}}=\phi_{\mathrm{b}} f
$$

for every $f \in L(G)$ invariant under $g \rightarrow k g k^{-1}$; the proof can be left to the reader. Of course these $f \in L(G)$ invariant under $g \rightarrow k g k^{-1}$ form a subalgebra $L^{\circ}(G)$ of $L(G)$, and it is easy to see that (31) is verified not only by the spherical functions (which in general do not belong to $L^{\circ}(G)$, because their carrier is not compact) but also by the functions belonging to the center of $L^{\circ}(G)$. Of course when $K$ reduces to the identity, (29) and (31) reduce to $\phi_{\mathfrak{b}}\left(g g^{\prime}\right)=\phi_{\mathfrak{b}}\left(g^{\prime} g\right)$, an obvious property of characters of compact groups.

So far we have not used the fact that the given representation $g \rightarrow T_{g}$ is irreducible, so that functional relations such as (29) do not characterize spherical functions. But this assumption will be essential in the next sections.

10. Spherical functions of type $b$ and characters of $L^{\circ}(\delta)$. In what follows, we shall denote by $L^{\circ}(G)$ the set of functions $f \in L(G)$ which are invariant under $g \rightarrow k g k^{-1}$; of course it is a subalgebra of $L(G)$, and the operator

$$
f(g) \rightarrow f^{\circ}(g)=\int f\left(k g k^{-1}\right) d k
$$

is a projection of $L(G)$ onto $L^{\circ}(G)$. We shall put

$$
L^{\circ}(\delta) \cap L(\delta) \cap L^{\circ}(G) ;
$$

this is also a subalgebra of $L(G)$, and $f \rightarrow f^{\circ}$ maps $L(\delta)$ onto $L^{\circ}(\mathfrak{d})$. Observe that for $f \in L^{\circ}(G)$ relation $\bar{\chi}_{\mathrm{b}} f=f$ implies $f \bar{\chi}_{\mathrm{b}}=f$ (for $f$ commutes with every measure $\epsilon_{k}$, hence with $\left.\bar{\chi}_{\triangleright}\right)$, so that $f \rightarrow \bar{\chi}_{b} f$ is a projection of $L^{\circ}(G)$ onto $L^{\circ}(\mathcal{b})$.

Of course, for every reasonable group algebra $A$, subalgebras $A^{\circ}$ and $A^{\circ}(\mathcal{b})$ can be defined in a similar way; we shall use them mainly in the case where $\boldsymbol{A}=\boldsymbol{M}(G)$ and in the case where $\boldsymbol{A}=\boldsymbol{A}_{\rho}(G)$ for some semi-norm $\rho$. If $\alpha$ is a measure, then $\alpha^{\circ}$ can be defined by the following "weak" integral:

$$
\alpha^{\circ}=\int \epsilon_{k} \alpha \epsilon_{k}^{-1} \cdot d k
$$

Observe that

$$
\left(\alpha^{\circ} \beta\right)^{\circ}=\left(\alpha \beta^{\circ}\right)^{\circ}=\alpha^{\circ} \beta^{\circ}
$$


for every $\alpha, \beta \in M(G)$.

We shall use the following general notation: given a continuous function $\phi$ on $G$ and a measure $\alpha \in M(G)$, we put

$$
\phi(\alpha)=\alpha(\phi)=\int \phi(g) d \alpha(g) ;
$$

if $d \alpha(g)=\theta(g) d g$ for some $\theta \in L(G)$, then we write $\phi(\theta)$ or $\theta(\phi)$. On the other hand, a function $\phi(g)$ on $G$ will be said to be quasi-bounded if there exists some semi-norm $\rho(g)$ such that

$$
\sup _{g \in G}|\phi(g)| / \rho(g)<+\infty ;
$$

it is obvious that every spherical function is quasi-bounded.

THEOREM 8. Let $\phi$ be a quasi-bounded continuous function on $G$, satisfying $\phi\left(k g k^{-1}\right)=\phi(g)$ and $\chi_{\diamond} \phi=\phi$. Then $\phi$ is a spherical function of type $\delta$ and height $p$ if and only if there exists a p-dimensional irreducible representation $f \rightarrow U_{f}$ of $L^{\circ}(\delta)$ such that

$$
\phi(f)=\operatorname{dim}(\boldsymbol{b}) \cdot \operatorname{Tr}\left(U_{f}\right)
$$

for every $f \in L^{\circ}(\boldsymbol{D})$.

This theorem establishes a strong connection between spherical functions of type $\delta$ and "characters" of the algebra $L^{\circ}(\delta)$. We shall prove it in several steps.

11. Proof of Theorem 8. Necessity of the condition. Consider a spherical function $\phi_{\mathfrak{b}}(g)=\operatorname{Tr}\left[E(\delta) T_{\mathfrak{g}}\right]$ of type $\delta$ and height $p$, and consider the representation $f \rightarrow \tilde{T}_{f}$ of $L(\delta)$ on $\mathfrak{S}(\delta)$; we know that this representation is irreducible and finite-dimensional.

LEMмA 9. The set of operators $\tilde{T}_{f}, f \in L^{\circ}(\delta)$, is the commutator of the representation $k \rightarrow T_{k}$ of $K$ on $\mathfrak{S}(\delta)$.

It is obvious that $f=f^{\circ}$ implies $T_{f} T_{k}=T_{k} T_{f}$. Conversely let $A$ be an operator on $\mathfrak{S}(\mathbb{D})$, and assume $A$ commutes with every $T_{k}$; since the representation $f \rightarrow \widetilde{T}_{f}$ of $L(\delta)$ is irreducible and finite-dimensional, there exists (Burnside's theorem) some $f \in L(\delta)$ such that $A=\tilde{T}_{f}$; but

$$
A=\int T_{k} A T_{k}^{-1} \cdot d k=\int T_{k} \tilde{T}_{f} T_{k}^{-1} \cdot d k=\tilde{T}_{f^{\circ}}
$$

by obvious computations, and this proves Lemma 9.

Now, since the representation $k \rightarrow T_{k}$ of $K$ on $\mathfrak{S}(\delta)$ decomposes into $p$ equivalent irreducible representations, it is clear that its commutator is isomorphic with the algebra $\boldsymbol{M}_{\boldsymbol{p}}$ of all $p \times p$ matrices; hence, by Lemma 9 , we can construct an isomorphism $\tilde{T}_{f} \rightarrow U_{f}$ of the algebra of operators $\tilde{T}_{f}, f$ 
$\in L^{\circ}(\delta)$, onto $M_{p}$; it is clear that $f \rightarrow U_{f}$ is a $p$-dimensional irreducible representation of $L^{\circ}(\mathfrak{D})$. On the other hand, it is known that the trace on $\boldsymbol{M}_{p}$ is characterized up to a constant factor by the fact that $\operatorname{Tr}(A B)=\operatorname{Tr}(B A)$; hence we have $\operatorname{Tr}\left(\widetilde{T}_{f}\right)=c \cdot \operatorname{Tr}\left(U_{f}\right)$, and actually $c=\operatorname{dim}(\mathfrak{D})$ as shown by taking $\widetilde{T}_{f}=1$; hence

$$
\phi_{\mathfrak{b}}(f)=f^{\prime} \phi_{\mathfrak{b}}(e)=\operatorname{Tr}\left[E(\mathfrak{\S}) T_{f}\right]=\operatorname{Tr}\left(\tilde{T}_{f}\right)=\operatorname{dim}(\mathfrak{\S}) \cdot \operatorname{Tr}\left(U_{f}\right)
$$

and this establishes the necessity of condition (39).

12. A lemma concerning $L(\delta)$. To prove the sufficiency of (39) we shall need a result which asserts that $L(\delta)$ can be deduced in a very simple way from $L^{\circ}(\mathfrak{D})$. Actually we shall need it for the similar subalgebras $A_{\rho}(\mathfrak{D})$ and $A_{\rho}^{\circ}(\mathfrak{D})$ of $A_{\rho}(G), \rho$ an arbitrary semi-norm.

First we prove:

Lemma 10. Let $\theta$ be a function or measure on $G$, satisfying $\chi_{\triangleright} \theta=\theta \chi_{\triangleright}=\theta$ for some $\mathfrak{b}$, and satisfying $\left(\epsilon_{k} \theta \epsilon_{h}\right)^{\circ}=0$ whenever $k, h \in K$; then $\theta=0$.

Proof. Denote by $\mathfrak{B}$ the vector space spanned by the measures $\alpha \theta \beta$ where $\alpha, \beta$ are arbitrary measures on $K$; by the first assumption about $\theta$ we may restrict ourselves to measures $\alpha$ and $\beta$ satisfying $\alpha \chi_{\triangleright}=\alpha$ and $\chi_{\triangleright} \beta=\beta$; but the set of such measures $\alpha$ is a finite-dimensional subspace in $\boldsymbol{M}(K)$; therefore, $\mathfrak{B}$ is finite-dimensional. Now define a linear representation $(k, h) \rightarrow T_{k, h}$ of $K \times K$ on $\mathfrak{B}$ by the formula $T_{k, h} \phi=\epsilon_{k} \phi \epsilon_{h^{-1}}$ for every $\phi \in \mathfrak{B}$; since $\chi_{\triangleright} \alpha=\alpha \chi_{\triangleright}$ for every measure $\alpha$ on $K\left(\chi_{b}\right.$ is a central function on $K$ ), it is clear that $\chi_{\triangleright} \phi \chi_{b}=\phi$ for every $\phi \in \mathfrak{B}$; using the identity $\chi_{\mathfrak{b}}=\int \epsilon_{k} \cdot \chi_{\mathfrak{b}}(k) d k$ (weak integral of measures on $K$ ), we see that

$$
\iint T_{k, h} \cdot \chi_{\mathfrak{b}}(k) \bar{\chi}_{\mathfrak{b}}(h) d k d h=1
$$

hence, if $\mathfrak{B}$ does not vanish, then the representation $k \rightarrow T_{k, k}$ of $K$ on $\mathfrak{B}$ has the character $\chi_{\mathfrak{b}}(k) \bar{\chi}_{b}(k)$; using a well known form of Schur's lemma (see, for instance, $[29$, p. 64]) we see that there exists a nonvanishing $\phi \in \mathfrak{B}$ such that $T_{k, k} \phi=\phi$, i.e., such that $\phi^{\circ}=\phi$; but from the fact that $\left(\epsilon_{k} \theta \epsilon_{h}\right)^{\circ}=0$ it follows more generally that $(\alpha \theta \beta)^{\circ}=0$ for arbitrary measures $\alpha, \beta$ on $K$, hence that $\phi \in \mathfrak{B}$ implies $\phi^{\circ}=0$, which contradicts the assumption that $\mathfrak{B} \neq 0$.

We can now prove our main lemma:

Lemma 11. For every semi-norm $\rho$, the linear combinations of translates under $K$ of functions belonging to $A_{\rho}^{\circ}(d)$ are everywhere dense in $A_{\rho}(\S)$.

Proof. Since $\epsilon_{k} \bar{\chi}_{\mathbb{\delta}}=\bar{\chi}_{\delta} \epsilon_{k}$, it is obvious that $A_{\rho}(\mathfrak{D})$ is invariant under translations by elements of $K$, so that every function $\epsilon_{k} f, k \in K, f \in A^{\circ}(\mathfrak{D})$, belongs to $A_{\rho}(\mathfrak{d})$. To prove that these functions span the whole of $A_{\rho}(\mathfrak{d})$ we shall use Hahn-Banach's theorem, so that we have only to prove that if a continuous linear form $f \rightarrow \theta(f)$ on $\boldsymbol{A}_{\rho}(\mathfrak{d})$ satisfies $\theta\left(\epsilon_{k} f\right)=0$ whenever $k \in K, f \in A^{\circ}(\mathfrak{D})$, then 
$\theta$ vanishes. But $f \rightarrow \theta(f)$ can be extended to a continuous linear form on the whole of $A_{\rho}(G)$-because $A_{\rho}(\mathfrak{D})$ is obviously a closed subspace of $A_{\rho}(G)$-so that there exists a measurable function $\theta(g)$ on $G$ such that $\theta(f)=\int f(g) \theta(g) d g$ for every $f \in A_{\rho}(\delta)$; of course we have a relation $|\theta(g)| \leqq M \cdot \rho(g)$. Setting $\theta^{\prime}$ $=\chi_{\diamond} \theta \chi_{\diamond}$ it is immediately seen that $\theta(f)=\theta^{\prime}(f)$ for every $f \in A_{\rho}(\mathfrak{b})$, so that we may assume that the function $\theta(g)$ satisfies $\chi_{\triangleright} \theta \chi_{b}=\theta$; then we have

$$
\theta\left(\bar{\chi}_{\triangleright} f \bar{\chi}_{\mathrm{b}}\right)=\theta(f)
$$

for every $f \in L(G)$; now if $f \in L(G)$ then $\left(\bar{\chi}_{b} f\right)^{\circ}=\bar{\chi}_{\delta} f^{\circ}$ belongs to $A_{\rho}^{\circ}(\mathcal{\delta})$ so that by our assumption about the linear form $f \rightarrow \theta(f)$ we get $\theta\left(\epsilon_{k} f^{\circ}\right)=0$ for every $f \in L(G)$, hence also $\theta\left(\epsilon_{k} f^{\circ} \epsilon_{h}\right)=0$ since $f^{\circ}$ commutes with $\epsilon_{k}$; setting $\theta_{1}=\left(\epsilon_{k} \theta \epsilon_{h}\right)^{\circ}$ we thus see that $\theta_{1}(f)=0$ for every $f \in L(G)$, so that $\theta_{1}=0$; applying Lemma 11 we get $\theta=0$ as asserted.

REMARK 8 . We implicitly used the identity

$$
\theta\left(f^{\circ}\right)=\theta^{\circ}(f),
$$

which can be easily proved.

13. Proof of Theorem 8. Sufficiency of the conditions. To prove the conditions stated in Theorem 8 are sufficient, we shall need some more lemmas.

Lemma 12. Let $\phi$ be a continuous function satisfying $\phi=\phi^{\circ}, \chi_{\triangleright} \phi=\phi$; then the following properties are equivalent:

$(\mathrm{a}): \phi(f g)=\phi(g f)$ for every $f, g \in L^{\circ}(\mathfrak{b})$;

(b) : $f^{\prime} \phi=\phi f^{\prime}$ for every $f \in L^{\circ}(\mathfrak{b})$.

Proof. Since $f^{\prime}(x)=f\left(x^{-1}\right)$, it is clear that $\phi(f)=f^{\prime} \phi(e)=\phi f^{\prime}(e)$; hence (a) is equivalent to $g^{\prime} f^{\prime} \phi(e)=f^{\prime} g^{\prime} \phi(e)=\phi f^{\prime} g^{\prime}(e)$, i.e., to $f^{\prime} \phi(g)=\phi f^{\prime}(g)$ for every $f, g \in L^{\circ}(\mathfrak{b})$; but since $f^{\prime} \phi$ and $\phi f^{\prime}$ are invariant under both $\theta \rightarrow \theta^{\circ}$ and $\theta \rightarrow \chi_{\diamond} \theta$, it is clear that (a) is equivalent to $f^{\prime} \phi(g)=\phi f^{\prime}(g)$ for arbitrary $f \in L^{\circ}(\mathfrak{b})$ and $g \in L(G)$, hence is equivalent to (b).

Lemma 13. Assume $\phi$ satisfies the assumptions of Theorem 8 and that $|\phi(g)| \leqq M \cdot \rho(g)$ for some semi-norm $\rho$; then we have $f^{\prime} \phi=\phi f^{\prime}$ for every $f \in A_{\rho}(\mathcal{(})$, and the set $\mathfrak{p}$ of $f \in A_{\rho}(\mathfrak{D})$ such that $f^{\prime} \phi=0$ is a regular two-sided ideal in $A_{\rho}(\mathfrak{b})$; furthermore, $f \in \mathfrak{p} \cap L^{\circ}(\mathfrak{D})$ if and only if $U_{f}=0$.

Proof. We have $\phi(f g)=\phi(g f)$ for every $f, g \in L^{\circ}(\mathcal{\delta})$, so that by Lemma 12 we get $f^{\prime} \phi=\phi f^{\prime}$ for every $f \in L^{\circ}(\mathfrak{D})$; but since $\phi=\phi^{\circ}$, we have $\epsilon_{k} \phi=\phi \epsilon_{k}$ and therefore the relation

$$
f^{\prime} \phi=\phi f^{\prime}
$$

is still true if $f=\epsilon_{k} h$ with $h \in L^{\circ}(\mathfrak{D})$; using Lemma 11 and the fact that $L^{\circ}(\delta)$ is everywhere dense in $A_{\rho}^{\circ}(\mathfrak{D})$ [this is due to the fact that $L(G)$ is everywhere dense in $A_{\rho}(G)$ and that $f \rightarrow \bar{\chi}_{\delta} f^{\circ}$ is a continuous projection of $A_{\rho}(G)$ onto $\left.A_{\rho}^{\circ}(\delta)\right]$ we conclude that (42) holds for every $f \in A_{\rho}(\delta)$. This of course proves that $\mathfrak{p}$ 
is a two-sided ideal in $A_{\rho}(\mathfrak{D})$. Now if $f \in L^{\circ}(\mathfrak{d}), f^{\prime} \phi=0$ is equivalent to $\phi(f g)=0$ for every $g \in L^{\circ}(\mathfrak{D})$, or to $\operatorname{Tr}\left(U_{f} U_{o}\right)=0$, and since the set of $U_{g}$ is a full matrix algebra, to $U_{f}=0$. Taking $u \in L^{\circ}(\delta)$ such that $U_{u}=1$ we see in a similar way that $u^{\prime} \phi=\phi u^{\prime}=\phi$, which implies that the ideal $\mathfrak{p}$ is regular.

We are now in a position to conclude the proof of Theorem 8 . Since $\mathfrak{p}$ is regular (and of course differs from $A_{\rho}(\delta)$ if $\phi \neq 0$ ), we can find a regular maximal left ideal $\mathfrak{a}$ in $\boldsymbol{A}_{\rho}(\mathfrak{d})$ containing $\mathfrak{p}$. Consider the corresponding ideal $\mathfrak{m}$ in $A_{\rho}(G)$, given by Lemma 8 , and consider the completely irreducible representation $g \rightarrow T_{g}$ of $G$ on the Banach space $\mathfrak{S}=A_{\rho}(G) / \mathfrak{m}$. If we denote by $f \rightarrow \boldsymbol{f}$ the natural mapping of $\boldsymbol{A}_{\rho}(G)$ onto $\mathfrak{S}$, then the operators $T_{\alpha}, \alpha \in \boldsymbol{M}(G)$, are obviously given by

$$
T_{\alpha} \boldsymbol{f}=\boldsymbol{f}^{\prime} \text { where } f^{\prime}=\alpha f ;
$$

in particular, the projection operator $E(\mathfrak{d})$ on $\mathfrak{S}$ is given by $E(\mathfrak{d}) \boldsymbol{f}=\boldsymbol{f}^{\prime}$ with $f^{\prime}=\bar{\chi} \mathrm{b} f$; but we know $($ Lemma 8$)$ that $f \bar{\chi}_{b} \equiv f(\bmod \mathfrak{m})$ for every $f$; hence $E(\mathfrak{d}) \boldsymbol{f}=\boldsymbol{f}^{\prime}$ with $f^{\prime}=\bar{\chi}_{\mathfrak{b}} f \bar{\chi}_{\mathfrak{b}}$, so that $f \rightarrow \boldsymbol{f}$ maps $\boldsymbol{A}_{\rho}(\mathfrak{d})$ onto $\mathfrak{S}(\mathfrak{D})$. Since $\mathfrak{a}=\mathfrak{m} \cap \boldsymbol{A}_{\rho}(\mathfrak{d})$, this obviously implies that the representation $f \rightarrow \widetilde{T}_{f}$ of $A_{\rho}(\mathfrak{b})$ on $\mathfrak{S}(\mathfrak{D})$ is equivalent to the natural representation $f \rightarrow V_{f}$ of $A_{\rho}(\mathfrak{d})$ on the factor space $A_{\rho}(\mathfrak{D}) / \mathfrak{a}$, which of course is finite-dimensional. If we denote by $\phi_{b}$ the spherical function of type $\delta$ of the representation $g \rightarrow T_{0}$ on $\mathfrak{S}$, we have therefore

$$
\phi_{\mathfrak{b}}(f)=\operatorname{Tr}\left(V_{f}\right)
$$

for every $f \in A_{\rho}(\mathfrak{D})$.

Since $\mathfrak{p}$ is a two-sided ideal in $\mathfrak{a}$, it is clear that $f \in \mathfrak{p}$ implies $V_{f}=0$; hence by the last assertion in Lemma 13 we see that, if $f \in L^{\circ}(\mathfrak{D})$, then $U_{f}=0$ implies $V_{f}=0$.

Now let $q$ be the height of $\phi_{b}$; by the direct part of the proof (no. 11) we know that there exists a $q$-dimensional irreducible representation $f \rightarrow W_{f}$ of $L^{\circ}(\mathfrak{d})$ such that $\phi_{\mathfrak{b}}(f)=\operatorname{dim}(\mathfrak{D}) \cdot \operatorname{Tr}\left(W_{f}\right)$ for every $f \in L^{\circ}(\mathfrak{b})$, and furthermore that $W_{f}=0$ is equivalent to $\widetilde{T}_{f}=0$, or to $V_{f}=0$; hence $U_{f}=0$ implies $W_{f}=0$, and since we are dealing with finite-dimensional irreducible representations of an associative algebra, we see that these two representations are equivalent. Hence $q=p$ and

$$
\phi(f)=\operatorname{dim}(\mathfrak{D}) \cdot \operatorname{Tr}\left(U_{f}\right)=\operatorname{dim}(\mathfrak{D}) \cdot \operatorname{Tr}\left(W_{f}\right)=\phi_{\mathrm{b}}(f),
$$

which proves that $\phi=\phi_{b}$.

COROLLARY OF THEOREM 8. The following properties are equivalent:

(a): $L^{\circ}(\mathfrak{b})$ is commutative;

(b): D occurs at most once in every completely irreducible representation of $G$.

In fact, if (b) holds, then $L^{\circ}(\mathfrak{D})$ has sufficiently many one-dimensional representations; conversely, if $L^{\circ}(\mathfrak{D})$ is commutative, then every finitedimensional irreducible representation of $L^{\circ}(\S)$ is one-dimensional, so that 
every spherical function of type $b$ is of height one.

It is easy to see that both properties (a) and (b) are equivalent to

(c): $L^{\circ}(\delta)$ is the center of $L(\delta)$.

First of all, it is clear that (c) implies (a). Now assume that $L^{\circ}(\delta)$ is commutative; then (b) holds, so that, for every completely irreducible representation $g \rightarrow T_{g}$ of $G$ on $\mathfrak{S}$, the representation $k \rightarrow T_{k}$ of $K$ on $\mathfrak{S}(\mathfrak{d})$ is irreducible; hence $\tilde{T}_{f}$ reduces to a scalar for every $f \in L^{\circ}(\mathfrak{D})$, so that we have $\widetilde{T}_{h f-f h}=0$ for every $f \in L^{\circ}(\delta)$ and every $h \in L(\delta)$; hence $L^{\circ}(\delta)$ is contained in the center of $L(\delta)$. Furthermore, if $f$ belongs to the center of $L(\delta)$, then it is clear that $\tilde{T}_{f}$ is a scalar in every completely irreducible representation of $G$, hence that $\widetilde{T}_{f}=\int T_{k} \widetilde{T}_{f} T_{k}^{-1} \cdot d k=\widetilde{T}_{f^{\circ}}$, which proves that $f=f^{\circ}$.

See a special case of the above result in [6].

14. Equivalence of representations. Let $g \rightarrow T_{g}$ be a completely irreducible representation of $G$ on $\mathfrak{S}$ and set $\rho(g)=\left\|T_{g}\right\|$; we shall say that the given representation is algebraically irreducible if the corresponding representation of $\boldsymbol{A}_{\rho}(G)$ is. As shown in $\$ 2$ every completely irreducible representation contains an algebraically irreducible representation.

The following result is a generalization of a theorem announced by HarishChandra in his first note [20]:

ThEOREM 9. Let $g \rightarrow T_{g}$ and $g \rightarrow T_{g}^{\prime}$ be two algebraically irreducible representations of $G$ such that $\left\|T_{o}\right\|=\left\|T_{o}^{\prime}\right\|$ for every $g \in G$; assume that, for some $\mathrm{b}$, their spherical functions of type $\mathfrak{d}$ are proportional and do not vanish; then these two representations are topologically equivalent.

Proof. By our assumption we have $\operatorname{Tr}\left(\tilde{T}_{f}\right)=c \cdot \operatorname{Tr}\left(\tilde{T}_{f}^{\prime}\right)$ for every $f \in L(\grave{D})$; hence the finite-dimensional irreducible representations $f \rightarrow \widetilde{T}_{f}$ and $f \rightarrow \widetilde{T}_{f}^{\prime}$ of $L(\mathfrak{D})$ are equivalent, so that we can find nonvanishing vectors $a \in \mathfrak{S}(\mathfrak{b})$ and $\boldsymbol{a}^{\prime} \in \mathfrak{S}^{\prime}(\mathfrak{b})$ such that $\tilde{T}_{f} \boldsymbol{a}=0$ is equivalent to $\tilde{T}_{f}^{\prime} \boldsymbol{a}^{\prime}=0$ (of course, $\mathfrak{S}$ and $\mathfrak{S}^{\prime}$ denote the representation spaces). Hence the maximal ideals $m: T_{f} a=0$, and $\mathrm{m}^{\prime}: T_{f}^{\prime} \mathbf{a}^{\prime}=0$ in $A_{\rho}(G)$, where $\rho(g)=\left\|T_{g}\right\|=\left\|T_{g}^{\prime}\right\|$, are identical (see Lemma 8), so that each representation is equivalent to the natural representation of $G$ on $A_{\rho}(G) / \mathfrak{m}$ - and is actually topologically equivalent to that representation, as follows from Banach's theorem about homomorphisms.

Theorem 9 does not apply to representations which are completely irreducible only; but if two such representations $g \rightarrow T_{g}$ and $g \rightarrow T_{0}^{\prime}$ on $\mathfrak{S}$ and $\mathfrak{S}^{\prime}$ have a common spherical function, and satisfy $\left\|T_{g}\right\|=\left\|T_{o}^{\prime}\right\|$ for every $g$, then the given representations become equivalent if we replace $\mathfrak{S}$ and $\mathfrak{S}^{\prime}$ by suitably choosen invariant subspaces; this follows in an obvious way from Theorem 9 and the results of $\$ 2$, no. 7 .

The case of unitary representations is much simpler; if two topologically irreducible unitary representations have a common spherical function, then they are unitarily equivalent. This was announced by Harish-Chandra in [20] and can be proved in a simple way. In fact, consider on $L(G)$ the involution 
$f \rightarrow f^{*}$ where $f^{*}(g)=\bar{f}\left(g^{-1}\right)$; clearly $L(\delta)$ and $L^{\circ}(\mathfrak{b})$ are invariant under this involution, and if we consider the representation $f \rightarrow \widetilde{T}_{f}$ of $L(\mathfrak{D})$ on the (finitedimensional) Hilbert space $\mathfrak{S}(\mathfrak{D})$, then we obviously have

$$
\tilde{T}_{f^{*}}=\left(\tilde{T}_{f}\right)^{*},
$$

so that we get a unitary representation of $L(\mathfrak{d})$ on $\mathfrak{S}(\mathfrak{d})$ in the sense of the general theory of unitary representations of algebras with an involution (see, for instance, [15] and [26]); now it is known and obvious that if two finitedimensional unitary irreducible representations of such an algebra are algebraically equivalent, then they are unitarily equivalent; hence if we use the notation in the proof of Theorem 9 we see that from the assumption of Theorem 9 follows the existence of nonvanishing vectors $a \in \mathfrak{S}(\mathfrak{d})$ and $\mathbf{a}^{\prime} \in \mathfrak{S}^{\prime}(\mathfrak{b})$ such that

$$
\left(\widetilde{T}_{f} \boldsymbol{a}, \boldsymbol{a}\right)=\left(\widetilde{T}_{f}^{\prime} \boldsymbol{a}^{\prime}, \boldsymbol{a}^{\prime}\right)
$$

for every $f \in L(\mathfrak{b})$; if we consider the positive-definite functions

$$
\theta(g)=\left(T_{g} \mathbf{a}, \boldsymbol{a}\right), \quad \theta^{\prime}(g)=\left(T_{g}^{\prime} \mathbf{a}^{\prime}, \boldsymbol{a}^{\prime}\right),
$$

we have therefore $\theta(f)=\theta^{\prime}(f)$ for every $f \in L(\delta)$; but it is obvious that $\chi_{\triangleright} \theta$ $=\theta \chi_{b}=\theta$ and the same is true for $\theta^{\prime}$, so that $\theta$ and $\theta^{\prime}$ are identical; since it is known that if two irreducible unitary representations of a group have a common "coefficient," then they are unitarily equivalent (see [13]), our assertion is proved.

REMARK 9. Of course, the proof of Theorem 9 requires only the trivial part of Theorem 8.

\section{Functional equation of spherical functions of height one.}

TheOREM 10. Let $\phi$ be a quasi-bounded continuous function on $G$; then $\phi$ is proportional to a spherical function of height one if and only if it satisfies

$$
\phi(e) \int \phi\left(k g k^{-1} g^{\prime}\right) d k=\phi(g) \phi\left(g^{\prime}\right)
$$

for arbitrary $g, g^{\prime} \in G$.

In fact, let $\phi_{b}$ be a spherical function of type $\mathfrak{b}$ and height one; setting $\phi=\phi_{\mathfrak{b}} / \operatorname{dim}(\mathfrak{d})$ it follows from Theorem 8 that $f \rightarrow \phi(f)$ is a homomorphism of $L^{\circ}(\mathfrak{d})$ onto the complex field, i.e.,

$$
\phi(f g)=\phi(f) \phi(g)
$$

for $f, g \in L^{\circ}(\mathfrak{D})$; now take arbitrary $f, g \in L(G)$ and compute $\phi\left(f^{\circ} g\right)$; by $\phi=\phi^{\circ}$ and $\chi_{\diamond} \phi=\phi$ we have

$$
\begin{aligned}
\phi\left(f^{\circ} g\right) & =\phi\left(\left(f^{\circ} g\right)^{\circ}\right)=\phi\left(f^{\circ} g^{\circ}\right)=\phi\left(\bar{\chi}_{\mathrm{b}} f^{\circ} g^{\circ} \bar{\chi}_{\mathrm{b}}\right) \\
& =\phi\left(\bar{\chi}_{\mathrm{b}} f^{\circ} \bar{\chi}_{\mathrm{b}} g^{\circ}\right)=\phi\left(\bar{\chi}_{\mathrm{b}} f^{\circ}\right) \phi\left(\bar{\chi}_{\mathrm{b}} g^{\circ}\right)=\phi(f) \phi(g)
\end{aligned}
$$


[we use the fact that $\bar{\chi}_{\delta} f^{\circ} \in L^{\circ}(\mathfrak{d})$ ]. Now since every measure with compact carrier can be approximated in an obvious sense by measures $f(x) d x$, we see that (47) more generally implies

$$
\phi\left(\alpha^{\circ} \beta\right)=\phi(\alpha) \phi(\beta)
$$

for every two $\alpha, \beta \in M(G)$; taking $\alpha=\epsilon_{g}, \beta=\epsilon_{g^{\prime}}$ we find at once (45).

Conversely, (45) means that (48) holds if $\alpha=\epsilon_{g}, \beta=\epsilon_{g^{\prime}}$; since every $\alpha \in \boldsymbol{M}(G)$ can be approximated by linear combinations of such measures, (45) implies (48) for arbitrary $\alpha, \beta \in \boldsymbol{M}(G)$, hence implies that, for every $\mathfrak{d}, f \rightarrow \phi(f)$ is the trace of a one- or null-dimensional representation of $L^{\circ}(\mathfrak{b})$; to prove that $\phi$ is proportional to a spherical function of type $\mathfrak{d}$ (for some $\mathfrak{b}$ ) it remains to prove that $\chi_{\triangleright} \phi=\phi$ for some $\mathfrak{b}$; but take $\mathfrak{b}$ such that $\chi_{\triangleright} \phi \neq 0$; since $\chi_{\triangleright} \in M^{\circ}(G)$, we have by $(48)$

$$
\phi\left(\bar{\chi}_{\triangleright} \alpha\right)=\phi\left(\bar{\chi}_{\diamond}\right) \phi(\alpha)
$$

for every $\alpha \in \boldsymbol{M}(G)$, i.e., $\chi_{\triangleright} \phi=\phi\left(\bar{\chi}_{\diamond}\right) \cdot \phi$; since $\chi_{\diamond} \chi_{\diamond}=\chi_{\triangleright}$, this implies $\phi\left(\bar{\chi}_{\triangleright}\right)^{2}$ $=\phi\left(\bar{\chi}_{b}\right)$, hence $=1$, and Theorem 10 is entirely proved.

REMARK 10. A special case of Theorem 10 has been announced by Gelfand in [6]; Gelfand studies only spherical functions associated with the onedimensional representation $k \rightarrow 1$ of $K$ and unitary representations of $G$. By Theorem 2, Theorem 10 applies (if $G$ is a semi-simple group with a faithful representation) to every $\phi_{b}$ if $\delta$ is an arbitrary one-dimensional representation of $K$; for instance, Theorem 10 applies to all spherical functions on the hyperbolic group (group of all real unimodular $2 \times 2$ matrices) because in this case $K$ is abelian. On the other hand, by the corollary of Theorem 1 , Theorem 10 applies also to all spherical functions on the Lorentz group. In $S L(n, C)$ with $n>2$, not all spherical functions are of height one; however it follows from Gelfand-Naimark's results that every irreducible component of the regular representation of $G$ has a spherical function of height one. In fact if we take the decomposition $G=K \cdot T, K \cap T=\Gamma$, which was used in the proof of Theorem 3 , and if $g \rightarrow T_{o}^{\alpha}$ is the representation induced by a one-dimensional representation $t \rightarrow \alpha(t)$ of $T$, then we have

$$
\text { multiplicity of } \mathfrak{b} \text { in } g \rightarrow T_{g}^{\alpha}=\text { multiplicity of } \gamma \rightarrow \alpha(\gamma) \text { in } \mathfrak{b} \text {; }
$$

hence if $\delta$ is the representation of $K$ whose "highest" weight is $\gamma \rightarrow \alpha(\gamma)$, we see that $b$ occurs exactly once in $g \rightarrow T_{\theta}^{\alpha}$, which proves our assertion.

Added in proof. The following result has been announced by Naimark (Doklady vol. 94 (1952) pp. 883-886): let $g \rightarrow T_{g}$ be any irreducible unitary representation of $S L(n, C)$; then some irreducible representation of $S U(n)$ is contained exactly once in $g \rightarrow T_{g}$.

Remark 11. Consider a semi-norm $\rho$; it is clear that the Banach space dual to $A_{\rho}(G)$ can be identified with the space $L_{\rho}^{\infty}(G)$ of measurable functions $\theta(g)$ on $G$ satisfying $\sup _{o \in G}|\theta(g)| / \rho(g)<+\infty$, the scalar product of $f \in A_{\rho}(G)$ 
and $\theta \in L_{\rho}^{\infty}(G)$ being given by $\int f(g) \theta(g) d g=\theta(f)$. Now for a given $b$ and a given $p$ denote by $\widehat{G}_{\rho}(\delta ; p)$ the set of spherical functions of type $\delta$ and height $p$ which belong to $L_{\rho}^{\infty}(G)$; it is easy to deduce from the proof of Theorem 8 that these spherical functions are associated with representations of $G$ on factor spaces $A_{\rho}(G) / \mathrm{m}$, hence can be obtained from representations $g \rightarrow T_{0}$ satisfying $\left\|T_{o}\right\| \leqq \rho(g)$; since

$$
|\phi(g)|=\left|\operatorname{Tr}\left[E(\delta) T_{\vartheta}\right]\right| \leqq \operatorname{dim} \mathfrak{g}(\delta) \cdot\left\|T_{\iota}\right\|,
$$

we see that every $\phi \in \widehat{G}_{\rho}(\mathcal{D} ; p)$ satisfies

$$
|\phi(g)| \leqq p \cdot \operatorname{dim}(\delta) \cdot \rho(g),
$$

so that $\widehat{G}_{\rho}(\mathfrak{D} ; p)$ is a bounded set in $L\left({ }_{\rho}^{\infty} G\right)$; hence the weak closure of $\widehat{G}_{\rho}(\mathfrak{b} ; p)$ in $L_{\rho}^{\infty}(G)$ is weakly compact.

Now consider $\widehat{G}_{\rho}(\mathfrak{D} ; 1)$; for every $\phi \in \widehat{G}_{\rho}(\emptyset ; 1), f \rightarrow \phi(f)$ is, up to a constant factor, a homomorphism of $L^{\circ}(G)$ into the complex field; hence the same is true for every weak limit of functions in $\widehat{G}_{\rho}(\mathfrak{D} ; 1)$ so that such a weak limit either vanishes or is proportional to some $\phi \in \widehat{G}_{\rho}(\mathbb{D} ; 1)$; actually it is practically obvious that the proportionality factor is 1 , and we see that the weak closure of $\widehat{G}_{\rho}(\mathfrak{b} ; 1)$ is obtained by adjoining to $\widehat{G}_{\rho}(\mathfrak{b} ; 1)$ the function 0 . Since this weak closure is weakly compact, we conclude that $\widehat{G}_{\rho}(\mathfrak{D} ; 1)$ is locally compact with respect to the weak topology on $L_{\rho}^{\infty}(G)$. By using the fact that $f \phi$ is proportional to $\phi$ for every $f \in L^{\circ}(G)$ and every $\phi \in \widehat{G}_{\rho}(\boldsymbol{b} ; 1)$ it can be shown that the weak topology actually reduces on $\widehat{G}_{\rho}(\mathfrak{b} ; 1)$ to the compact-open topology (uniform convergence on every compact set); the proof is identical, up to trivial modifications, with that of the similar property of the character group of an abelian group.

It is very probable that similar results hold for arbitrary $\widehat{G}_{\rho}(\delta ; p)$. The case of spherical functions corresponding to unitary representations of $G$ will be studied in our second paper.

16. Integral formulas for spherical functions. From the point of view of practical applications it would be extraordinarily important to get integral representations of spherical functions, generalizing the well known formulas for Bessel's and Legendre's functions. Important results were announced by Harish-Chandra, and so far we are unable to prove (and a fortiori to improve!) these results by using "functional" methods.

Assume that $G$ has a closed subgroup $T$ such that every $g$ has at least one representation $g=k \cdot t$, and set $K \cap T=\Gamma$. For a given one-dimensional representation $t \rightarrow \alpha(t)$ of $T$, consider the induced representation

$$
g \rightarrow T_{g}^{\alpha}
$$

of $G$; we want to compute the functions

$$
\phi_{b}^{\alpha}(g)=\operatorname{Tr}\left[E(\delta) T_{g}^{\alpha}\right]
$$


which are spherical functions if $g \rightarrow T_{g}^{\alpha}$ is irreducible $\left({ }^{13}\right)$.

To do this, we recall that the representation space $\mathfrak{S E}^{\alpha}$ of this induced representation is the set of continuous functions $\theta(g)$ such that $\theta(t g)=\alpha(t) \theta(g)$, and that $T_{g}^{\alpha} \theta\left(g^{\prime}\right)=\theta\left(g^{\prime} g\right)$. Of course $\mathfrak{S}^{\alpha}$ can be identified with the set of functions $\theta \in L(K)$ such that $\theta(\gamma k)=\alpha(\gamma) \theta(k)$. Now to compute $\phi_{0}^{\alpha}$ we shall compute for every $f \in L(G)$ the number $\phi_{b}^{\alpha}(f)=\int \phi_{b}^{\alpha}(g) f(g) d g=\operatorname{Tr}\left[E(\delta) T_{f}^{\alpha}\right]$. First we have

$$
T_{f}^{\alpha} \theta(k)=\int \theta(k g) f(g) d g=\int \theta(g) f\left(k^{-1} g\right) d g ;
$$

let $k \rightarrow \dot{k}$ be the natural mapping of $K$ onto $K / \Gamma$; by the theory of homogeneous spaces we have an integration formula

$$
\int f(g) d g=\int d \dot{h} \int f(t h) d t
$$

where $d t$ is a left-invariant measure on $T$; hence (51) yields

$$
T_{f}^{\alpha} \theta(k)=\int d \dot{h} \int \theta(t h) f\left(k^{-1} t h\right) d t=\int d \dot{h} \int \theta(h) f\left(k^{-1} t h\right) \alpha(t) d t ;
$$

but since $\theta(\gamma h)=\alpha(\gamma) \theta(h)$, it is immediately seen that, for every $k$, the function $\int \theta(h) f\left(k^{-1} t h\right) \alpha(t) d t$ is invariant under $h \rightarrow \gamma h$, so that we may write

$$
T_{f}^{\alpha} \theta(k)=\iint \theta(h) f\left(k^{-1} t h\right) \alpha(t) d t d h ;
$$

now, to compute $E(\mathfrak{b}) T_{f}^{\alpha}$ we observe that $E(\mathfrak{b})$ is given by $\theta(k) \rightarrow \int \theta(k l) \bar{\chi}_{\mathbb{\delta}}(l) d l$, so that we get by standard computations

$$
E(\mathfrak{b}) T_{f}^{\alpha} \theta(k)=\iiint \theta(h) f\left(l k^{-1} t h\right) \alpha(t) \chi_{\mathfrak{\delta}}(l) d t d h d l ;
$$

in other words, $E(\delta) T_{f}^{\alpha}$ is an integral operator on $\mathfrak{S}^{\alpha}$, defined by the kernel

$$
K_{f}^{\alpha}(k, h)=\iint f\left(l k^{-1} t h\right) \alpha(t) \chi_{\mathfrak{b}}(l) d t d l .
$$

(13) Observe that the irreducibility of $g \rightarrow T_{a}^{\alpha}$ is not a necessary condition for the function $\phi_{\mathrm{b}}^{\alpha}$ to be spherical (this is due to the fact that the converse of Lemma 3 in $\$ 1$ is not true); for instance, if $b$ occurs exactly once in $g \rightarrow T_{\sigma}^{\alpha}$, then $\phi_{b}^{\alpha}$ is a spherical function (for in this case the operators $\tilde{T}_{f}^{\alpha}, f \in L^{\circ}(\delta)$, commute with the $T_{k}$ on $\mathfrak{S}^{\alpha}(\delta)$, hence reduce to scalar operators, so that $\phi_{\delta}^{\alpha}$ satisfies the functional equation of spherical functions of type $\delta$ and height one). In the general case, it is very probable that every $\phi_{b}^{\alpha}$ is a finite sum of spherical functions of type $b$ (this is true at least when $g \rightarrow T_{0}^{\alpha}$ is unitary with respect to the scalar product $\int \theta(k) \bar{\theta}^{\prime}(k) d k$ on $L(K)$, for in this case the representation $f \rightarrow \widetilde{T}_{f}^{\alpha}$ of $L(\delta)$ on $\mathfrak{S}^{\alpha}(\delta)$ is unitary, hence is completely decomposable into a finite sum of irreducible representations). 
To compute the trace of this operator on $\mathfrak{S}^{\alpha}$, we first observe that $T_{f}^{\alpha}$ can be defined, by using the kernel (55), on the whole of $L(K)$; now if we consider on $L(K)$ the operator

$$
P(\alpha): \theta(k) \rightarrow \int \theta(\gamma k) \bar{\alpha}(\gamma) d \gamma,
$$

it is obvious that $P(\alpha)$ is a projection of $L(K)$ onto $\mathfrak{S}^{\alpha}$; on the other hand we have by (55) the relations

$$
K_{f}^{\alpha}(\gamma k, h)=\alpha(\gamma) \cdot K_{f}^{\alpha}(k, h) ; \quad K_{f}^{\alpha}(k, \gamma h)=\alpha(\gamma)^{-1} \cdot K_{f}^{\alpha}(k, h)
$$

so that $P(\alpha) T_{f}^{\alpha}=T_{f}^{\alpha} P(\alpha)=T_{f}^{\alpha}$; hence the trace of $T_{f}^{\alpha}$ on $\mathfrak{S}^{\alpha}$ is identical with the trace of $T_{f}^{\alpha}$ on $L(K)$, so that

$$
\begin{aligned}
\phi_{\delta}^{\alpha}(f) & =\int K_{f}^{\alpha}(h, h) d h=\iiint f\left(k h^{-1} t h\right) \alpha(t) \chi_{\mathbb{b}}(k) d t d k d h \\
& =\iiint f(k t h) \alpha(t) \chi_{\triangleright}(k h) d t d k d h .
\end{aligned}
$$

Since $d t$ is invariant under $t \rightarrow t \gamma,(56)$ yields

$$
\begin{aligned}
\phi_{\delta}^{\alpha}(f) & =\iiint \int f(k t \gamma h) \alpha(t) \alpha(\gamma) \chi_{\triangleright}(h k) d t d k d h d \gamma \\
& =\iiint \int f(k t h) \alpha(t) \bar{\alpha}(\gamma) \chi_{\triangleright}(\gamma h k) d t d k d h d \gamma ;
\end{aligned}
$$

now define a function $\chi_{b}^{\alpha}(g)$ on $G$ by the requirement that

$$
\chi_{\delta}^{\alpha}(t h)=\alpha(t) \int \bar{\alpha}(\gamma) \chi_{\mathbb{\delta}}(\gamma h) d \gamma ;
$$

it is immediately seen that the second member of (58) depends only on $t$, although the representation $g=t h$ may be not unique; then (57) leads to

$$
\phi_{\mathbf{b}}^{\alpha}(f)=\iiint f(k t h) \chi_{\mathbf{b}}^{\alpha}(t h k) d t d k d h,
$$

hence, by using the theory of homogeneous spaces, to

$$
\phi_{\mathrm{b}}^{\alpha}(f)=\iint f(k g) \chi_{\mathrm{b}}^{\alpha}(g k) d g d k=\int f(g) d g \int \chi_{\mathrm{b}}^{\alpha}\left(k g k^{-1}\right) d k ;
$$

since $\phi_{b}^{\alpha}(f)=\int \phi_{b}^{\alpha}(g) f(g) d g$, we conclude that

$$
\phi_{0}^{\alpha}(g)=\int \chi_{0}^{\alpha}\left(k g k^{-1}\right) d k
$$


where $\chi_{b}^{\alpha}$ is given by (58).

It is probable that in most cases all spherical functions are given by formulas similar to (61); in semi-simple groups, this was announced by Harish-Chandra for the spherical functions associated with one-dimensional representations of $K$; in case $G=S L(2, C), K=S U(2)$ the fact that all spherical functions associated with the representation $k \rightarrow 1$ of $K$ are given by (61) was also proved in a very interesting way by Naimark in [26]; Naimark's proof essentially uses the functional equation of these spherical functions.

When $\delta$ is the identity representation $k \rightarrow 1$ of $K$, formulas (58) and (61) take on a simpler form; in fact, if we use the decomposition $G=N \cdot K$ of $G$ with $T=\Gamma \cdot N$, then (58) proves that we must assume $\alpha(\gamma)=1$ in order that $\chi_{\delta}^{\alpha}$ be $\neq 0$, and then we get $\chi_{\delta}^{\alpha}(t h)=\alpha(t)$, i.e., $\chi_{\delta}^{\alpha}(n k)=\alpha(n)$; hence (61) leads to

$$
\phi_{b}^{\alpha}(g)=\int \alpha(k g) d k \text { where } \alpha(n k)=\alpha(n) .
$$

This formula can be used in order to study the structure of the algebra $L(\delta)$. In fact it is clear that $L(\delta)$ is the set of functions $f \in L(G)$ such that $f\left(k g k^{\prime}\right)$ $=f(g)$; since $\delta$ is a one-dimensional representation of $K$, we know that $L(\delta)$ is a commutative algebra; furthermore if we set

$$
\hat{f}(\alpha)=\int f(g) \phi_{b}^{\alpha}(g) d g,
$$

then we know that $f \rightarrow \hat{f}(\alpha)$ is a homomorphism of $L(\delta)$ onto the complex field, and also that we get in this way sufficiently many such homomorphisms (because by Lemmas 5 and 7 we know that the induced representations $g \rightarrow T_{g}^{\alpha}$ of $G$ form a complete set); hence (63) establishes an isomorphism between $L(\delta)$ and an algebra of functions on the group of characters of $N$.

Observe that (63) can be computed in the following way. Let $Z$ be the topological commutator group of $N$ and assume there exists an abelian closed subgroup $\Delta$ of $N$ such that $N=\Delta \cdot Z$ (which arises in all practical situations); we have $\alpha(\delta z)=\alpha(\delta)$; hence

$$
\begin{aligned}
\widehat{f}(\alpha) & =\iint f(g) \alpha(k g) d k d g=\iint f\left(k^{-1} g\right) \alpha(g) d k d g=\int f(g) \alpha(g) d g \\
& =\iint f(n k) \alpha(n k) d n d k=\int f(n) \alpha(n) d n=\iint f(\delta z) \alpha(\delta z) d \mu(\delta) d z
\end{aligned}
$$

i.e.,

$$
\hat{f}(\alpha)=\int \dot{f}(\delta) \alpha(\delta) d \mu(\delta)
$$


where we set

$$
\dot{f}(\delta)=\int f(\delta z) d z
$$

and where $d \mu(\delta)$ denotes a Haar measure an $\Delta$.

It is clear that $\dot{f}$ is a continuous function with compact carrier on $\Delta$ for every $f \in L(\delta)$; hence $f \rightarrow \dot{f}$ is a mapping of $L(\mathfrak{d})$ onto a subset $\dot{A}$ of $L(\Delta)$; by (63) and (64) it is obvious that $f \rightarrow \dot{f}$ is actually an isomorphism of the algebra $L(\delta)$ (equipped with the convolution product on $G$ ) onto a subalgebra of $L(\Delta)$ (equipped with the convolution product on $\Delta$ ). To prove HarishChandra's theorem, namely that (62) represents all spherical functions of type $\mathfrak{D}(k \rightarrow 1)$ we thus see that we should establish that every homomorphism of $\dot{A}$ onto the complex field can be extended to a homomorphism of the whole of $L(\Delta)$. A proof of this by the methods of functional analysis would be extremely interesting; it is very probable that such a proof would require a detailed analysis of $\dot{A}$ and mainly an "inner" characterization of the functions $\dot{f}(\delta)$ which can be obtained by (65). This is connected with a study of integral equations (65); if $G=S L(2, R)$, it can be shown that (65) is essentially equivalent to the classical Abel integral equation, so that it seems that a study of more general groups would lead to similar integral equations in several variables. This question is also connected with the explicit form of Plancherel's theorem for spherical functions; actually if an explicit inversion formula is known for (65), i.e., if we can express $f$ in terms of $\dot{f}$, then by (64) and the Fourier inversion formula on $\Delta$ we can express $f$ in terms of $\hat{f}$ and this is just the Plancherel theorem for functions belonging to $L(\mathcal{b})$ !

A complete answer to these problems will be given elsewhere for $G=S L(2, R)$. Now we shall study the case of motion groups, which is much simpler than the case of semi-simple groups. In that case, integral formulas can be obtained for all spherical functions as we shall prove.

17. The case of motion groups. First of all, for a given $b$ denote by $k \rightarrow U_{k}$ an irreducible representation of $K$ on a vector space $\mathfrak{E}$, and assume this representation belongs to $\bar{D}$; defining for an arbitrary operator $A$ on $\mathbb{E}$ the number $\sigma(A)=\operatorname{dim}(\mathbb{E}) \cdot \operatorname{Tr}(A)$ we see that

$$
\bar{\chi}_{\triangleright}(k)=\sigma\left(U_{k}\right) \text {; }
$$

now let $f \in L^{\circ}(\delta)$; since $\bar{\chi}_{\triangleright} f=f$, we have

$$
f(g)=\int f\left(k^{-1} g\right) \bar{\chi}_{\mathfrak{b}}(k) d k=\int \sigma\left(U_{k}\right) f\left(k^{-1} g\right) d k ;
$$

hence

$$
f(g)=\sigma|F(g)|
$$


where $F$ is the operator-valued function

$$
F(g)=\int U_{k}^{-1} \cdot f(k g) d k
$$

using the fact that $f=f^{\circ}$ it is obvious that $F$ satisfies

$$
F(k g h)=U_{k} F(g) U_{h}
$$

for arbitrary $k, h \in K$. Of course, $F$ is continuous and vanishes outside some compact set. Now we want to express the convolution product in $L^{\circ}(\mathfrak{D})$ in terms of the corresponding functions (68); if $F_{1}$ and $F_{2}$ correspond to functions $f_{1}, f_{2} \in L^{\circ}(\mathcal{\delta})$, then the function $F_{1} F_{2}$ corresponding to $f_{1} f_{2}$ is given by

$$
\begin{aligned}
F_{1} F_{2}(n) & =\int U_{k}^{-1} \cdot f_{1} f_{2}(k n) d k=\iint U_{k}^{-1} f_{1}(k n g) f_{2}\left(g^{-1}\right) d k d g \\
& =\iiint U_{k}^{-1} \cdot f_{1}\left(k n n^{\prime} h\right) f_{2}\left(h^{-1} n^{\prime-1}\right) d k d h d n^{\prime} ;
\end{aligned}
$$

but it follows from the orthogonality relations of characters that

$$
\int U_{k}^{-1} \cdot \sigma\left(U_{k} A\right) d k=A
$$

for every operator $A$ on $\&$; hence

$$
\begin{aligned}
F_{1} F_{2}(n) & =\iiint U_{k}^{-1} \cdot \sigma\left[U_{k} F_{1}\left(n n^{\prime}\right) U_{h}\right] \cdot \sigma\left[U_{h}^{-1} F_{2}\left(n^{\prime-1}\right)\right] d k d h d n^{\prime} \\
& =\iint F_{1}\left(n n^{\prime}\right) U_{h} \cdot \sigma\left[U_{h}^{-1} F_{2}\left(n^{\prime-1}\right)\right] d h d n^{\prime}
\end{aligned}
$$

so that we finally obtain

$$
F_{1} F_{2}(n)=\int F_{1}\left(n n^{\prime}\right) F_{2}\left(n^{\prime-1}\right) d n^{\prime} .
$$

Of course this is true even if $N$ is neither abelian nor invariant.

We shall now use the fact that $N$ is an abelian invariant subgroup. Denote by $L_{\mathfrak{E}}(N)$ the set of all operator-valued functions $F(n)$ which are continuous and vanish outside some compact set in $N$; under the convolution product $(71), L_{\mathbb{E}}(N)$ is an algebra; furthermore it is clear by $(69)$ that $L^{\circ}(\mathfrak{D})$ is isomorphic with the subalgebra of those functions $F \in L_{\mathfrak{E}}(N)$ which satisfy

$$
F\left(k n k^{-1}\right)=U_{k} F(n) U_{k}^{-1} .
$$

To find spherical functions of type $\delta$, we have to construct all finite-dimen- 
sional irreducible representations of $L^{\circ}(\mathcal{D})$ satisfying obvious continuity conditions. But define

$$
F^{\circ}(n)=\int U_{k}^{-1} F\left(k n k^{-1}\right) U_{k} \cdot d k
$$

for every $F \in L_{\mathfrak{E}}(N)$; of course we get a projection of $L_{\mathfrak{E}}(N)$ onto $L^{\circ}(\mathfrak{\wp})$, with the properties

$$
\left(F^{\circ} G\right)^{\circ}=\left(F G^{\circ}\right)^{\circ}=F^{\circ} G^{\circ}
$$

now let $\mathfrak{a}$ be a regular maximal left ideal in $L^{\circ}(\mathfrak{b})$, and consider the set $\mathfrak{m}$ of $F \in L_{\mathfrak{E}}(N)$ such that $(G F)^{\circ} \in \mathfrak{a}$ for every $G \in L_{\mathfrak{G}}(N)$; it is obvious that $\mathfrak{m}$ is a left-ideal, and by using (74) it is easy to see that every right identity modulo $a$ is also a right identity modulo $\mathfrak{m}$, so that $\mathfrak{m}$ is regular; furthermore we have, by (74), $\mathfrak{a} \subset \mathfrak{m}$ so that $\mathfrak{a}=\mathfrak{m} \cap L^{\circ}(\mathfrak{b})$ by the maximality of $\mathfrak{a}$. Now since $\mathfrak{m}$ is regular, it is contained in a maximal left ideal; hence we see that $\mathfrak{a}$ is the intersection of $L^{\circ}(\mathfrak{d})$ with some regular maximal left ideal in $L_{\mathbb{E}}(N)$; from this it follows at once that every irreducible representation of $L^{\circ}(\mathfrak{b})$ is contained in some irreducible representation of $L_{\mathfrak{E}}(N)$. But $L_{\mathfrak{E}}(N)$ is isomorphic with the tensor product of the algebra of operators on $\&$ and the algebra $L(N)$ of complex-valued continuous functions with compact carrier on $N$; using the fact that every "reasonable" irreducible representation of $L(N)$ is one-dimensional and given by $f \rightarrow \hat{f}(\alpha)=\int f(n) \alpha(n) d n$, where $n \rightarrow \alpha(n)$ is a character of $N$, we easily see that every irreducible representation of $L_{\mathfrak{E}}(N)$ is given by

$$
F \rightarrow \widehat{F}(\alpha)=\int F(n) \alpha(n) d n .
$$

Therefore, every irreducible representation of $L^{\circ}(\delta)$ is contained in a representation (75), i.e., is obtained as an irreducible component of a representation (75) restricted to $L^{\circ}(\delta)$.

Of course it may happen that $(75)$ is still irreducible on $L^{\circ}(\mathfrak{b})$; in that case it is quite easy to compute the corresponding spherical function $\left({ }^{14}\right)$. Actually, this function $\phi(g)$ is given (Theorem 8 ) by

$$
\phi(f)=\operatorname{dim}(\mathfrak{d}) \cdot \operatorname{Tr}[\widehat{F}(\alpha)]=\operatorname{dim}(\mathbb{E}) \cdot \operatorname{Tr}[\widehat{F}(\alpha)]=\sigma[F(\alpha)]
$$

for every $f \in L^{\circ}(\delta)$; using (67) we get

$$
\phi(f)=\int f(n) \alpha(n) d n
$$

for every $f \in L^{\circ}(\mathcal{D})$. Now for an arbitrary $f \in L(G)$ we have

${ }^{(14)}$ Observe that the integral formulas we are going to prove yield all spherical functions in case $K$ is abelian (and, even in this case, lead to nontrivial "special" functions). 


$$
\begin{aligned}
\phi(f) & =\phi\left(\bar{\chi}_{\triangleright} f^{\circ}\right)=\int \bar{\chi}_{\triangleright} f^{\circ}(n) \alpha(n) d n \\
& =\iiint f\left(h k n h^{-1}\right) \chi_{\triangleright}(k) \alpha(n) d n d k d h ;
\end{aligned}
$$

using $\chi_{b}(k h) \doteq \chi_{\triangleright}(h k)$ and the fact that $d n$ is invariant under $n \rightarrow h n h^{-1}$ we find at once

$$
\phi(f)=\int f(g) \phi(g) d g=\iint f(n k) \chi_{\diamond}(k) \alpha\left(h n h^{-1}\right) d n d k d h
$$

and, since $f \in L(G)$ is arbitrary, we see that

$$
\phi(n k)=\chi_{0}(k) \int \alpha\left(h n h^{-1}\right) d h ;
$$

of course this is a special case of formulas in the preceding section.

It may happen also that representation (75) is not irreducible on $L^{\circ}(\mathrm{b})$, and then the above method breaks down; for instance, if the character $\alpha$ is invariant under the mappings $n \rightarrow k n k^{-1}$, then it is easy to see that the operators $\hat{F}(\alpha), f \in L^{\circ}(\delta)$, commute with the $U_{k}$, hence reduce to scalar operators, so that in this case the representation (75) of $L^{\circ}(\delta)$ is not irreducible [but it turns out that $(80)$ is still valid up to a constant factor, due to the fact that $\widehat{F}(\alpha)$ is identical, up to a constant factor, with the scalar operator $\sigma[\widehat{F}(\alpha)] \cdot 1$, so that (76) is still true ]. However, let $\mathfrak{F} \subset$ \& be a minimal subspace invariant under the operators $\widehat{F}(\alpha), f \in L^{\circ}(\delta)$; the representation $f \rightarrow \widehat{F}(\alpha)$ of $L^{\circ}(\mathbb{D})$ on $\mathfrak{F}$ is irreducible, hence defines a spherical function on $G$. To compute this spherical function $\phi$, we choose an orthonormal basis $\left(e_{i}\right), 1 \leqq i \leqq p$, of $\mathfrak{F}$, and we get by Theorem 8

$$
\left.\phi(f)=\operatorname{dim}(\boldsymbol{\delta}) \cdot \sum \widehat{F}(\alpha) \boldsymbol{e}_{i}, \boldsymbol{e}_{i}\right)=\operatorname{dim}(\delta) \cdot \sum \int\left(F(n) \boldsymbol{e}_{i}, \boldsymbol{e}_{i}\right) \alpha(n) d n
$$

now for arbitrary $f \in L(G)$ set $f^{\prime}=\bar{\chi}_{\diamond} f^{\circ}$; then the corresponding function $F^{\prime}(n)$ is given [see equation (68)] by

$$
F^{\prime}(n)=\int U_{k}^{-1} \cdot f^{\prime}(k n) d k=\iiint U_{k}^{-1} \cdot f\left(h l k n h^{-1}\right) \chi_{\mathfrak{b}}(l) d k d h d l
$$

so that we get (up to a constant factor)

$$
\begin{aligned}
\phi(f)=\phi\left(f^{\prime}\right) & =\sum \iiint \int\left(\boldsymbol{e}_{i}, U_{k} \mathbf{e}_{i}\right) f\left(h l k n h^{-1}\right) \chi_{\downarrow}(l) \alpha(n) d n d k d h d l \\
& =\sum \iiint \int\left(U_{h} U_{l} \mathbf{e}_{i}, U_{k} \mathbf{e}_{i}\right) f\left(k n h^{-1}\right) \chi_{\triangleright}(l) \alpha(n) d n d k d h d l ;
\end{aligned}
$$


but from $\bar{\chi}_{\mathrm{d}}(l)=\sigma\left(U_{l}\right)$ it follows that

$$
\int U_{l} \cdot \chi_{\triangleright}(l) d l=1
$$

up to a constant factor, so that

$$
\begin{aligned}
\phi(f) & =\sum \iiint\left(U_{h} \mathbf{e}_{i}, U_{k} \mathbf{e}_{i}\right) f\left(k n h^{-1}\right) \alpha(n) d n d k d h \\
& =\sum \iiint\left(U_{k}^{-1} U_{h} U_{k} \boldsymbol{e}_{i}, \mathbf{e}_{i}\right) f\left(k n k^{-1} h^{-1}\right) \alpha(n) d n d k d h \\
& =\sum \iiint\left(U_{k}^{-1} U_{h}^{-1} U_{k} \mathbf{e}_{i}, \boldsymbol{e}_{i}\right) f(n h) \alpha\left(k^{-1} n k\right) d n d k d h
\end{aligned}
$$

and since $f \in L(G)$ is arbitrary, we finally obtain

$$
\phi(n h)=\int \theta\left(k h k^{-1}\right) \alpha\left(k n k^{-1}\right) d k
$$

where the function $\theta$ is given by

$$
\theta(h)=\sum\left(\boldsymbol{e}_{i}, U_{h} \boldsymbol{e}_{i}\right)=\sum \overline{\left(U_{h} \boldsymbol{e}_{i}, \boldsymbol{e}_{i}\right)},
$$

i.e., is a sum of coefficients of the irreducible representation $b$ (we recall that $k \rightarrow U_{k}$ belongs to $\bar{\delta}$ ).

Of course, if $\theta$ denotes an arbitrary sum of coefficients of $\delta$, then (85) does not necessarily represent a spherical function; we may only assert that every spherical function of type $\mathfrak{b}$ is obtained by an integral formula (85).

Observe that (85) proves that

$$
\phi(n) / \phi(e)=\int \alpha\left(k n k^{-1}\right) d k
$$

does not depend on $\mathfrak{b}$; formula (87) could be obtained in another way, namely by using a functional property of spherical functions (which, unfortunately, works for motion groups only). Let $g \rightarrow T_{g}$ be a completely irreducible representation of $G$ on a Banach space $\mathfrak{S}$; for every $n$, the operator $\int T_{k} T_{n} T_{k}^{-1} d k$ commutes with the $T_{k}$; on the other hand, since $k n k^{-1} \in N$ and since $N$ is abelian, this operator commutes with every $T_{n^{\prime}}$ too-hence with every $T_{q}$ hence reduces to a scalar:

$$
\int T_{k} T_{n} T_{k}^{-1} \cdot d k=\lambda(n) \cdot 1 ;
$$

if we consider the spherical function of type $\delta$ of the given representation, $\phi_{\mathrm{b}}(g)=\operatorname{Tr}\left[E(\mathfrak{d}) T_{g}\right]$, we have therefore 


$$
\int \phi\left(g k n k^{-1}\right) d k=\int \operatorname{Tr}\left[E(\mathfrak{b}) T_{g} T_{k} T_{n} T_{k}^{-1}\right] d k=\lambda(n) \phi(g) ;
$$

taking $g=e$ we find $\phi(n)=\lambda(n) \phi(e)$, and this proves that every spherical function $\phi$ on $G$ satisfies

$$
\phi(e) \int \phi\left(g k n k^{-1}\right) d k=\phi(g) \phi(n) .
$$

Now if we replace $\phi$ by $\phi / \phi(e)$, and if we set

$$
\phi(f)=\int f(n) \phi(n) d n ; \quad f^{\circ}(n)=\int f\left(k n k^{-1}\right) d k
$$

for every $f \in L(N)$, it is obvious that (90) implies

$$
\phi\left(f_{1}^{\circ} f_{2}\right)=\phi\left(f_{1}\right) \phi\left(f_{2}\right)
$$

for arbitrary $f_{1}, f_{2} \in L(N)$; hence $\phi$ defines a "character" of the subalgebra $L^{\circ}(N)$; but by using as above relations (74) it is easy to see that every character of $L^{\circ}(N)$ can be extended to a character of $L(N)$, and this leads to (87).

The most classical instance of (87) is obviously given by the Bessel function $J_{0}$; but other interesting functions can be obtained; for instance consider in the complex euclidean 3 -space $N$ the compact abelian group of rotations represented by matrices

$$
k=\left(\begin{array}{lll}
e^{i \phi} & 0 & 0 \\
0 & e^{i \psi} & 0 \\
0 & 0 & e^{-i \phi-i \psi}
\end{array}\right)
$$

if we consider the motion group $G$ generated by these rotations and the translations on $N$, then we see that the spherical functions are given on $N$ by

$$
(2 \pi)^{-2} \iint_{0}^{2 \pi} \exp \left(\alpha x e^{i \phi}+\beta \bar{x} e^{-i \phi}+\gamma y e^{i \psi}+\delta \bar{y} e^{-i \psi}+\lambda z e^{-i \phi-i \psi}+\mu \bar{z} e^{i \phi+i \psi}\right) d \phi d \psi
$$

where $\alpha, \cdots, \mu$ are arbitrary complex numbers, so that they can be expressed in terms of the function

$$
\begin{array}{r}
(2 \pi)^{-2} \iint_{0}^{2 \pi} \exp (i[x \cdot \cos \phi+y \cdot \sin \phi+z \cdot \cos \psi+t \cdot \sin \psi \\
+u \cdot \cos (\phi+\psi)+v \cdot \sin (\phi+\psi)]) \cdot d \phi d \psi \\
=(2 \pi)^{-1} \int_{0}^{2 \pi} J_{0}\left(\left((x+u \cdot \cos \psi+v \cdot \sin \psi)^{2}+(y-u \cdot \sin \psi+v \cdot \cos \psi)^{2}\right)^{1 / 2}\right) \\
\cdot \exp (i(z \cdot \cos \psi+t \cdot \sin \psi)) d \psi
\end{array}
$$


which cannot be expressed in terms of Bessel's functions (which means that the author is unable to do it).

This and other similar examples have been studied by J. Delsarte in unpublished papers.

\section{Differential Properties of SPherical FunCtions}

18. The algebra $\mathrm{D}(G)$. In this section, we assume that $G$ is a connected Lie group. In addition to functions and measures, we may consider distributions on $G$ in the sense of L. Schwartz [28]; a distribution $\alpha$ on $G$ is a linear form $\alpha(f)$ on the space of indefinitely differentiable functions with compact carrier on $G$, satisfying certain continuity conditions - see [28]. It is known that every distribution $\alpha$ has a carrier: it is the smallest closed set $S \subset G$ such that $\alpha(f)=0$ whenever $f$ vanishes in some neighbourhood of $S$. If the carrier of $\alpha$ is a compact set, then $\alpha(f)$ can be defined for arbitrary indefinitely differentiable functions $f$, without any restriction concerning the carrier of $f$. We shall denote by $\boldsymbol{D}(G)$ the vector space of all distributions with compact carrier on $G$; we have therefore "natural" inclusions

$$
L(G) \subset \boldsymbol{M}(G) \subset \boldsymbol{D}(G) .
$$

We shall use for distributions the same notation as for measures, namely $\alpha(f)=f(\alpha)=\int f(g) d \alpha(g)$; this apparently involves some inconvenience (e.g., to denote differential operators by integral formulas), but actually these inconveniences are advantages, just because we intend to deduce differential properties from integral ones!

As in euclidean spaces, it is possible to define a convolution product on $D(G)$; if $\alpha, \beta \in D(G)$, then the distribution $\gamma=\alpha \beta$ is given by

$$
\int f(z) d \gamma(z)=\iint f(x y) d \alpha(x) d \beta(y)
$$

for every indefinitely differentiable function $f$ on $G$. Of course $D(G)$ is an associative algebra under that product. We also note that a convolution product $\alpha_{1} \alpha_{2} \cdots \alpha_{n}$ can be defined whenever all factors but one have compact carriers, and that the associative rule still holds in this case; see [28, vol. II, p. 14].

We shall denote by $U(G)$ the set of $\alpha \in D(G)$ whose carrier reduces to the identity. Of course, $U(G)$ is a subalgebra of $D(G)$. If we denote by

$$
\left(x_{1}, \cdots, x_{n}\right)
$$

a system of coordinates in a neighbourhood of $e$, then every $\alpha \in U(G)$ is a finite linear combination of the derivatives

$$
f \rightarrow \frac{\partial^{p_{1}+\ldots+p_{n} f}}{\partial x_{1^{1}}^{p_{1}} \cdots \partial x_{n^{n}}^{p^{n}}}(e)
$$


where the $p_{i}$ are arbitrary non-negative integers $\left({ }^{15}\right)$, and conversely. In particular denote by $\&$ the set of linear combinations of the distributions

$$
f \rightarrow \frac{\partial f}{\partial x_{i}}(e)
$$

then it is easy to see that $\boldsymbol{g}$ is isomorphic to the complexification of the Lie algebra of $G$, the Jacobi product in $\xi$ being given by $[\alpha, \beta]=\alpha \beta-\beta \alpha$ (convolution products!). One can deduce from this (L. Schwartz, unpublished) that $\boldsymbol{U}(G)$ is isomorphic to the universal associative enveloping algebra of $\boldsymbol{g}$-but we shall not use this fact.

The elements of the (abstract) algebra $U(G)$ can be represented by differential operators on $G$. In fact, for a given $\alpha \in D(G)$ and an indefinitely differentiable function $f$ on $G$, the convolution product $\alpha f$ is an indefinitely differentiable function (see [28, vol. II, p. 22]), given by

$$
\alpha f(x)=\int f\left(y^{-1} x\right) d \alpha(y) ;
$$

we shall denote by $X_{\alpha}$ the operator $f \rightarrow \alpha f$; it is clear that $X_{\alpha}$ commutes with the right translations on $G$, and if $\alpha \in U(G)$, we obviously obtain a differential operator in the classical sense. Conversely, let $f \rightarrow X f$ be a differential operator commuting with right translations; there exists one and only one distribution $\alpha$ such that

$$
\alpha(f)=X f^{\prime}(e),
$$

and since $f \rightarrow X f$ commutes with right translations, we get $X f=\alpha f$; of course, $\alpha \in U(G)$, and finally the algebra $U(G)$ is isomorphic to the algebra of all differential operators commuting with right translations.

We shall also use for distributions the operations $\alpha \rightarrow \alpha^{\prime}$ and $\alpha \rightarrow \alpha^{\circ} ; \alpha^{\prime}$ is the transform of $\alpha$ under $x \rightarrow x^{-1}$, and $\alpha^{\circ}$ is given by

$$
\alpha^{\circ}=\int \epsilon_{k} \alpha \epsilon_{k^{-1}} \cdot d k
$$

Of course we have

$$
\alpha(f)=f^{\prime} \alpha(e)=f \alpha^{\prime}(e)=\alpha^{\prime} f(e)=\alpha f^{\prime}(e)
$$

for every indefinitely differentiable $f$; and

$$
\alpha^{\circ}(f)=\alpha\left(f^{\circ}\right)
$$

under the same assumption.

We shall denote by $D^{\circ}(G)$ the set of $\alpha \in D(G)$ such that $\alpha^{\circ}=\alpha$, and we $\boldsymbol{U}(G)$.

(15) If all $p_{i}$ vanish, then we get the distribution $\epsilon: f \rightarrow f(e)$, which is the unit element of 
shall set $U^{\circ}(G)=D^{\circ}(G) \cap U(G)$. Of course $D^{\circ}(G)$ and $U^{\circ}(G)$ are subalgebras of $D(G)$ and $U(G)$. We shall use the following property:

LEMma 14. Let $f$ be an analytic function on $G$, invariant under $K$; then $f=0$ is equivalent to

$$
f(\alpha)=0 \quad \text { for every } \quad \alpha \in U^{\circ}(G) .
$$

Proof. Since $f$ is analytic and since $G$ is connected, it is clear that $f=0$ is equivalent to $f(\alpha)=0$ for every $\alpha \in U(G)$; since $f=f^{\circ}$ and since $f(\alpha)=f\left(\alpha^{\circ}\right)$, this proves the lemma.

We shall also use a topology on $D^{\circ}(G)$ :

Definition. We say that a variable $\alpha \in D^{\circ}(G)$ converges to a given $\alpha_{0}$ $\in D^{\circ}(G)$ if $f(\alpha)$ converges to $f\left(\alpha_{0}\right)$ for every analytic function $f$ satisfying $f=f^{\circ}$.

This topology is entirely different from that of L. Schwartz, but is much more useful than L. Schwartz's for studying analytic functions; this is mainly due to the following property:

Lemma 15. $U^{\circ}(G)$ is everywhere dense in $D^{\circ}(G)$.

Proof. By the Hahn-Banach theorem we have only to prove that if a continuous linear form $\alpha \rightarrow f(\alpha)$ on $D^{\circ}(G)$ vanishes on $U^{\circ}(G)$, then it vanishes on the whole of $D^{\circ}(G)$; but by the "weak duality" theory (see [4]) such a linear form is defined by an analytic function on $G$ invariant under $K$, so that Lemma 15 follows from Lemma 14.

To conclude these preliminary remarks, we recall some properties of elliptic differential operators (actually we shall consider only very special instances of that concept, so that we shall state these properties in the special situation which we are interested in). Let $\alpha \in U(G)$ and consider the corresponding operator $X_{\alpha} f=\alpha f$. Assume that, in a given coordinate system around $e, \alpha$ is given by a relation

$$
\alpha(f)=a \cdot f(e)+\sum a_{i} \cdot \frac{\partial f}{\partial x_{i}}(e)+\sum a_{i j} \frac{\partial^{2} f}{\partial x_{i} \partial x_{j}}(e)
$$

where the coefficients $a_{i j}$ form a strictly positive-definite matrix. Then it is easy to see that in every analytic coordinate system around an arbitrary point of $G$ we have a representation

$$
X_{\alpha} f(x)=a(x) f(x)+\sum a_{i}(x) \frac{\partial f}{\partial x_{i}}(x)+\sum a_{i j}(x) \frac{\partial^{2} f}{\partial x_{i} \partial x_{j}}(x)
$$

where the coefficients are analytic and where, for every $x$, the matrix $\left(a_{i j}(x)\right)$ is strictly positive-definite. Using a classical theorem due to S. Bernstein we conclude that every indefinitely differentiable function $f$ satisfying a relation

$$
X_{\alpha} f=\lambda f+g,
$$


where $\lambda$ is a scalar and where $g$ is an analytic function, is itself analytic $\left({ }^{16}\right)$. This implies that every solution of an equation $\left(X_{\alpha}-\lambda\right)^{n} f=0$ is analytic; in fact, if we assume the theorem is already proved for $n-1$ and if we set $g=\left(X_{\alpha}-\lambda\right) f$, then $g$ is analytic since we have $\left(X_{\alpha}-\lambda\right)^{n-1} g=0$; and since $f$ satisfies $X_{\alpha} f=\lambda \cdot f+g$ we see that $f$ is itself analytic, so that our assertion is proved by induction.

We observe that there exist distributions $\alpha \in U(G)$ satisfying the above conditions and $\alpha=\alpha^{\circ}$. To prove this it is enough to prove that if $\alpha$ satisfies the above conditions, then $\alpha^{\circ}$ does. But for a given $k$ put $y=k x k^{-1}$ and define $\rho_{i j}=\partial y_{i} /\left.\partial x_{j}\right|_{x=e}$; then it is clear that the matrix $\left(b_{i j}\right)$ associated with the distribution $\epsilon_{k} \alpha \epsilon_{k^{-1}}$ is given by $b_{i j}=\sum a_{p q} \rho_{p i} \rho_{g j}$, i.e., is strictly positive-definite; since a (continuous) sum of strictly positive-definite matrices is still strictly positive-definite, our assertion is proved. Finally we get the following basic result:

Lemma 16. There exists a distribution $\alpha \in U^{\circ}(G)$ such that every function $f$ satisfying a relation $\left(X_{\alpha}-\lambda\right)^{n} f=0$ is analytic.

19. Analytic vectors in irreducible representations. Let $g \rightarrow T_{g}$ be a representation of $G$ on some Banach space $\mathfrak{S}$. We shall denote by $\mathfrak{S}_{\infty}$ the set of finite linear combinations of vectors $T_{f} a$, where $\boldsymbol{a}$ is arbitrary in $\mathfrak{S}$ and where $f$ is an arbitrary indefinitely differentiable function with compact carrier on $G$. This "Gårding subspace" (see [5]) is everywhere dense in $\mathfrak{S}$ and invariant under the $T_{g}$. Since the convolution of an indefinitely differentiable function with compact carrier and an arbitrary distribution is an indefinitely differentiable function, it is clear that for every $\boldsymbol{a} \in \mathfrak{S}_{\infty}$ and every $\boldsymbol{b} \in \mathfrak{S}^{\prime}$ the corresponding "coefficient" $\left(T_{g} \boldsymbol{a}, \boldsymbol{b}\right)$ is indefinitely differentiable. As proved by Gårding, we may associate with every $\alpha \in D(G)$ a linear operator $T_{\alpha}$ on $\mathfrak{S}_{\infty}$, given by

$$
\left(T_{\alpha} \boldsymbol{a}, \boldsymbol{b}\right)=\int\left(T_{\theta} \boldsymbol{a}, \boldsymbol{b}\right) d \alpha(g), \quad \boldsymbol{a} \in \mathfrak{S}_{\infty}, \boldsymbol{b} \in \mathfrak{S}^{\prime}:
$$

in general $T_{\alpha}$ is not bounded, but it is easy to see that $\alpha \rightarrow T_{\alpha}$ is a representation of the algebra $D(G)$ on $\mathfrak{S}_{\infty}$; if $\alpha$ is a function or a measure, $T_{\alpha}$ reduces of course on $\mathfrak{S}_{\infty}$ to the (already defined) operator $T_{\alpha}$, and is bounded.

Lemma 17. Assume that $\mathfrak{S}(\mathfrak{b})$ is finite-dimensional for some $\mathfrak{b}$; then for every $\mathbf{a} \in \mathfrak{S}(\mathfrak{b})$ and every $\boldsymbol{b} \in \mathfrak{S}^{\prime}$ the function $\left(T_{g} \boldsymbol{a}, \boldsymbol{b}\right)$ is analytic on $G$.

Proof. Since $\mathfrak{S}_{\infty}$ is invariant under the operators $T_{\alpha}$, it is invariant under the projection operator $E(\mathfrak{D})$; since $\mathfrak{S}_{\infty}$ is everywhere dense in $\mathfrak{S}, E(\mathfrak{D}) \mathfrak{S}_{\infty}$ is everywhere dense in $\mathfrak{S}(\mathfrak{d})$, hence is identical with $\mathfrak{S}(\mathfrak{b})$ since $\operatorname{dim} \mathfrak{S}(\mathfrak{d})<+\infty$;

${ }^{(16)}$ Of course, "analytic" means: analytic with respect to the real parameters on $G$, even when $G$ has a complex structure. 
therefore, we see that $\mathfrak{S}(\mathfrak{D}) \subset \mathfrak{S}_{\infty}$, so that we may apply every $T_{\alpha}, \alpha \in D(G)$, to every $a \in \mathfrak{S}(\mathfrak{D})$. Now take the distribution $\alpha$ of Lemma 16; since $\alpha=\alpha^{\circ}$, it is clear that $T_{\alpha}$ commutes with the $T_{k}$, so that $T_{\alpha} \mathfrak{S}(\mathfrak{d}) \subset \mathfrak{S}(\mathfrak{D})$; since $\mathfrak{S}(\mathfrak{d})$ is finite-dimensional, we can find a basis $\left(\boldsymbol{e}_{i}\right)$ of $\mathfrak{S}(\mathfrak{d})$ such that every $\boldsymbol{e}_{i}$ satisfies a relation $\left(T_{\alpha}-\lambda\right)^{n} \boldsymbol{e}_{i}=0$; setting $\theta(g)=\left(T_{g} \boldsymbol{e}_{i}, b\right), b \in \mathfrak{S}^{\prime}$, we see that $\theta^{\prime}$ is an eigenfunction of $\left(X_{\alpha}-\lambda\right)^{n}$, hence is analytic, and this obviously proves Lemma 17.

In what follows, we shall say that a vector $a \in \mathfrak{E}$ is analytuc if the function $\left(T_{o} a, b\right)$ is analytic for every $b \in \mathfrak{S}^{\prime}$; this definition is somewhat weaker than that of a "well-behaved" vector due to Harish-Chandra; but for vectors belonging to $\mathfrak{S}_{\infty}$ the two definitions are practically identical.

THEOREM 11. Let $g \rightarrow T_{\theta}$ be a topologically irreducible representation of $G$ on $\mathfrak{S}$, and assume that $0<\operatorname{dim} \mathfrak{S}(\mathfrak{b})<+\infty$ for some $\mathfrak{\delta}$; then analytic vectors are everywhere dense in $\mathfrak{S}$.

Proof. The set of analytic vectors is an invariant subspace, so that Theorem 11 follows from Lemma 17.

Corollary. Assume that every irreducible representation of $K$ is contained a finite number of times only in every completely irreducible representation of $G$; then every spherical function is analytic.

We can now state our main result, which will be proved in the next sections and is an "infinitesimal" counterpart to Theorem 8 of $\$ 3$.

THEOREM 12. Let $\phi$ be a quasi-bounded analytic function on $G$, invariant under $K$; then $\phi$ is proportional to a spherical function of height $p$ if and only if there exists a p-dimensional irreducible representation $\alpha \rightarrow U_{\alpha}$ of $U^{\circ}(G)$ such that

$$
\phi(\alpha)=c \cdot \operatorname{Tr}\left(U_{\alpha}\right)
$$

for every $\alpha \in U^{\circ}(G)$; if $\phi$ is a spherical function of type $\delta$, then $c=\operatorname{dim}(\delta)$.

On semi-simple Lie groups, this result was known to Harish-Chandra (who did not explicitely publish it in his notes); Harish-Chandra's method of proof apparently reduces to constructing more or less explicitly the function satisfying (100), and in this method the assumption that $\phi$ is quasi-bounded is not useful; we shall need this assumption in order to be able to use Theorem 8 , and also because we do not know whether it can be avoided in not necessarily semi-simple groups. From the practical point of view, this assumption is not very important; the main problem is to represent spherical functions (more accurately, solutions of (100)) by integral formulas, and if this is done, then it is not difficult to verify that these functions correspond to representations of $G$ on Banach spaces!

20. Proof of Theorem 12. Direct part. Let $g \rightarrow T_{0}$ be a completely irreducible representation of $G$ on a Banach space $\mathfrak{S}$; consider the correspond- 
ing representation $\alpha \rightarrow T_{\alpha}$ of $D(G)$ on the Gårding subspace $\mathfrak{S}_{\infty}$; we recall that $\mathfrak{S}_{\infty}$ contains every $\mathfrak{S}(\mathfrak{d})$ (of course, we assume that $\operatorname{dim} \mathfrak{S}(\mathfrak{d})<+\infty$ for every $\mathfrak{D})$. If $\alpha \in D^{\circ}(G)$, then every $\mathfrak{S}(\mathfrak{D})$ is invariant under $T_{\alpha}$, so that $T_{\alpha}$ induces on every $\mathfrak{S}(\mathfrak{D})$ an operator $\widetilde{T}_{\alpha}(\mathcal{(})$; of course, $\alpha \rightarrow \widetilde{T}_{\alpha}(\mathfrak{d})$ is a representation of $D^{\circ}(G)$ on $\mathfrak{S}(\mathfrak{d})$. It is clear that every $\tilde{T}_{\alpha}(\mathfrak{d})$ commutes with every $T_{k}$; since $D^{\circ}(G)$ contains $L^{\circ}(G)$ and since the set of operators $\tilde{T}_{f}(\mathcal{D}), f \in L^{\circ}(G)$, is the commutator of the representation $k \rightarrow T_{k}$ of $K$ on $\mathfrak{S}(\delta)$ (see Lemma 9), we see that the set of operators $\widetilde{T}_{\alpha}(\delta), \alpha \in D^{\circ}(G)$, is the commutator of the representation $k \rightarrow T_{k}$ of $K$ on $\mathfrak{S}(\mathfrak{D})$. We shall prove a much stronger property:

Lemma 18. The commutator of the representation $k \rightarrow T_{k}$ of $K$ on $\mathfrak{S}(\mathfrak{d})$ is the set of operators $\widetilde{T}_{\alpha}(\mathfrak{b}), \alpha \in U^{\circ}(G)$.

Since we are dealing with finite-dimensional sets of operators, we have only to prove that the set of $\tilde{T}_{\alpha}(\delta), \alpha \in U^{\circ}(G)$, is everywhere dense in the set of $\widetilde{T}_{\alpha}(\delta), \alpha \in D^{\circ}(G)$; hence Lemma 18 will follow from Lemma 15 provided we can prove that the representation $\alpha \rightarrow \widetilde{T}_{\alpha}(\mathfrak{b})$ of $D^{\circ}(G)$ on $\mathfrak{S}(\mathfrak{b})$ is continuous, which means that for every $a \in \mathfrak{S}(\mathfrak{b})$ and every $b \in \mathfrak{S}^{\prime}\left({ }^{17}\right)$ the linear form $\alpha$ $\rightarrow \theta(\alpha)=\left(\widetilde{T}_{\alpha}(\boldsymbol{\delta}) \boldsymbol{a}, \boldsymbol{b}\right)=\left(T_{\alpha} \boldsymbol{a}, \boldsymbol{b}\right)=\int\left(T_{g} \boldsymbol{a}, \boldsymbol{b}\right) d \alpha(g)$ is continuous on $D^{\circ}(G)$. If we define $\theta(g)=\left(T_{g} a, b\right)$, we have $\theta(\alpha)=\int \theta(g) d \alpha(g)=\int \theta^{\circ}(g) d \alpha(g)$ since $\alpha=\alpha^{\circ}$; hence our assertion is equivalent to the fact that the function $\theta^{\circ}(\mathrm{g})$ is analytic on $G$. But by the proof of Lemma 17 we can write $\theta=\theta_{1}+\cdots+\theta_{q}$, where every $\theta_{i}$ is an eigenfunction of an elliptic operator invariant under $K$; of course, the functions $\theta_{i}^{\circ}$ are also eigenfunctions of such operators, hence are analytic, and this concludes the proof of Lemma 18.

We can now prove the necessity of (100). For every $\alpha \in D(G)$ we have $\phi_{\mathrm{b}}(\alpha)=\operatorname{Tr}\left[E(\mathfrak{b}) T_{\alpha}\right]$, in particular $\phi_{\mathrm{b}}(\alpha)=\operatorname{Tr}\left[\tilde{T}_{\alpha}(\mathfrak{\delta})\right]$ for every $\alpha \in U^{\circ}(G)$; but by Lemma 18 the set of operators $\widetilde{T}_{\alpha}(\delta), \alpha \in U^{\circ}(G)$, is isomorphic with the algebra of all $p \times p$ matrices (where $p$ is the height of the spherical function $\left.\phi_{0}\right)$, so that the existence of the representation $\alpha \rightarrow U_{\alpha}$ is proved as in no. 11 .

21. Proof of Theorem 12. Sufficiency of the condition. We now consider a function $\phi$ satisfying the assumptions of Theorem 12 ; to prove that $\phi$ is proportional to a spherical function, we shall establish that it satisfies the assumptions of Theorem 8 , so that we have to replace the given representation of $U^{\circ}(G)$ by a representation of some $L^{\circ}(\delta)$. This will be done in several steps.

Lemma 19. $U_{\alpha}=0$ is equivalent to $\alpha^{\prime} \phi=0$.

Proof. Define $\theta=\alpha^{\prime} \phi$; since $f \rightarrow \alpha^{\prime} f$ is a differential operator with analytic coefficients, $\theta$ is analytic, and satisfies $\theta=\theta^{\circ}$ since both $\alpha$ and $\phi$ do; hence (Lemma 14) $\theta=0$ is equivalent to $\theta(\beta)=0$ for every $\beta \in U^{\circ}(G)$, or to $\phi(\alpha \beta)$ $=0$, or to $\operatorname{Tr}\left(U_{\alpha} U_{\beta}\right)=0$, and since the set of operators $U_{\beta}$ is a full matrix

(17) $\mathfrak{S}^{\prime}$ denotes the Banach space dual to $\mathfrak{S}$. 
algebra (Burnside's theorem), Lemma 19 is proved.

Lemma 20. Let $\mathfrak{B}_{\phi}$ be the set of functions $\alpha \phi, \alpha \in U^{\circ}(G)$; then $\mathfrak{B}_{\phi}$ is a $p^{2}$ dimensional vector space.

Proof. Obvious by Lemma 19 and the fact that $U^{\circ}(G)$ is invariant under $\alpha \rightarrow \alpha^{\prime}$.

Lemma 21. $\beta \phi$ and $\phi \beta$ are analytic functions for every $\beta \in D(G)$.

Proof. We have $\phi \in \mathfrak{B}_{\phi}$ since $U^{\circ}(G)$ contains $\epsilon$; now consider the distribution $\alpha \in U^{\circ}(G)$ of Lemma $16 ; \mathfrak{B}_{\phi}$ is invariant under the differential operator $X_{\alpha}$; since $\phi \in \mathfrak{B}_{\phi}$, we can therefore decompose $\phi$ into a finite sum of eigenfunctions of operators $\left(X_{\alpha}-\lambda\right)^{n}$; but (associativity of the convolution product when all factors but one have compact carriers) if $\theta$ is an eigenfunction of $\left(X_{\alpha}-\lambda\right)^{n}$, the same is true for $\theta \beta$, whenever $\beta \in D(G)$; this of course proves that $\phi \beta$ is analytic; the same proof applies to $\beta \phi$.

Lemma 22. $\mathfrak{B}_{\phi}$ is identical with the set of functions $\alpha \phi, \alpha \in D^{\circ}(G)$, and also with the set of functions $f \phi, f \in L^{\circ}(G)$.

Proof. Denote by $\mathfrak{B}$ the space of all continuous functions on $G$ with the topology of pointwise convergence; $\mathfrak{B}$ is a locally convex topological vector space satisfying Hausdorff's axiom, so that every finite-dimensional subspace of $\mathfrak{B}$ is closed in $\mathfrak{B}$; in particular $\mathfrak{B}_{\phi}$ is closed in $\mathfrak{B}$. Now consider the mapping $\alpha \rightarrow \alpha \phi$ of $D^{\circ}(G)$ into $\mathfrak{B}$; this mapping is continuous because if $g \in G$, then the linear form $\alpha \rightarrow \alpha \phi(g)=\int \theta\left(g^{\prime}\right) d \alpha\left(g^{\prime}\right)$ [where $\theta=\left(\epsilon_{g}^{\prime} \alpha\right)^{\circ} \phi$ is analytic by Lemma 21 and invariant under $K$ ] is continuous on $D^{\circ}(G)$. Hence (Lemma 15) $\mathfrak{B}_{\phi}$ is everywhere dense in the set of functions $\alpha \phi, \alpha \in D^{\circ}(G)$, which proves the first part of Lemma 22. To prove the second part we have only to use similar arguments and to observe that $L^{\circ}(G)$ is everywhere dense in $D^{\circ}(G)$, which is obvious by Hahn-Banach's theorem.

Lemma 23. $\alpha \phi=\phi \alpha$ for every $\alpha \in D^{\circ}(G)$.

Proof. Consider the case where $\alpha \in U^{\circ}(G)$; for every $\beta \in U^{\circ}(G)$ we have $\alpha \phi(\beta)=\beta^{\prime} \alpha \phi(e)=\phi\left(\alpha^{\prime} \beta\right)=\operatorname{Tr}\left(U_{\alpha^{\prime}} U_{\beta}\right)$ and in the same way $\phi \alpha(\beta)$ $=\operatorname{Tr}\left(U_{\beta} U_{\alpha^{\prime}}\right)$; hence $\alpha \phi=\phi \alpha$ by Lemma 14. The general case follows from Lemma 15 and the fact that $\alpha \phi$ and $\phi \alpha$ depend continuously on $\alpha \in D^{\circ}(G)$ (see the proof of Lemma 22).

Lemma 24. There exists a character $\chi_{\triangleright}$ of $K$ such that $\chi_{\triangleright} \phi=\phi$.

Proof. By Péter-Weyl's theorem, there exists a character $\chi$ of $K$ such that $\chi \phi \neq 0$; since $\chi=\chi^{\circ}$, we have $\chi \in D^{\circ}(G)$, so that (Lemma 22) there exists some $\alpha \in U^{\circ}(G)$ such that $\chi \phi=\alpha \phi$; for every $\beta \in U^{\circ}(G)$ we have $\chi \beta=\beta \chi$ (for $\beta$ commutes with every $\epsilon_{k}$, hence with $\chi=\int \epsilon_{k} \cdot \chi(k) d k$, too), so that by using Lemma 23 we get $\beta \alpha \phi=\beta \chi \phi=\chi \beta \phi=\chi \phi \beta=\alpha \phi \beta=\alpha \beta \phi$; hence (Lemma 
19) the operator $U_{\alpha^{\prime}}$ commutes with every $U_{\beta}$, i.e. reduces to a scalar; this implies $\chi \phi=c \cdot \phi$ for some number $c$, and since $\chi \chi=\chi$ we finally obtain $\chi \phi=\phi$.

Conclusion of the proof. Since $\chi_{\diamond} \phi=\phi$, we have $f^{\prime} \phi=f^{\prime} \chi_{\diamond} \phi=g^{\prime} \phi$, where $g=\bar{\chi}_{b} f$; hence, by Lemma $22, \mathfrak{B}_{\phi}$ is identical with the set of functions $f^{\prime} \phi, f \in L^{\circ}(\mathfrak{d})$; we can therefore associate with every $f \in L^{\circ}(\mathfrak{d})$ an operator $U_{f}$ by choosing an $\alpha \in U^{\circ}(G)$ such that $f^{\prime} \phi=\alpha^{\prime} \phi$ and by setting $U_{f}=U_{\alpha}$; although $\alpha$ may be not unique, $U_{\alpha}$ is unique by Lemma 19. It is clear that $f \rightarrow U_{f}$ is a linear mapping of $L^{\circ}(\mathcal{D})$ onto the algebra of all $p \times p$ matrices; in addition from $f^{\prime} \phi=\alpha^{\prime} \phi$ and $g^{\prime} \phi=\beta^{\prime} \phi$ it follows by using Lemma 23 that $f^{\prime} g^{\prime} \phi=f^{\prime} \phi g^{\prime}=\alpha^{\prime} \phi g^{\prime}=\alpha^{\prime} g^{\prime} \phi=\alpha^{\prime} \beta^{\prime} \phi$, so that $f \rightarrow U_{f}$ is a representation of $L^{\circ}(\mathfrak{D})$. Since $f^{\prime} \phi=\alpha^{\prime} \phi$ implies $\phi(f)=f^{\prime} \phi(e)=\alpha^{\prime} \phi(e)=\phi(\alpha)=c \cdot \operatorname{Tr}\left(U_{\alpha}\right)=c \cdot \operatorname{Tr}\left(U_{f}\right)$ we see that $\phi$ satisfies the assumptions of Theorem 8 , so that the proof of Theorem 12 is now complete.

22. Another formulation of Theorem 12. Let $A$ be an associative algebra over an arbitrary field $\Omega$; let $\mathfrak{S}$ be a vector space over $\Omega$, and assume we are given:

(a) a linear representation $f \rightarrow U_{f}$ of $\boldsymbol{A}$ on $\mathfrak{S}$;

(b) a linear representation $f \rightarrow V_{f}$ of the opposite algebra $\left({ }^{18}\right)$ on $\mathfrak{S}$; then we shall say that $\{\mathfrak{S}, U, V\}$ is a double representation of $A$ if we have

$$
U_{f} V_{g}=V_{g} U_{f}
$$

for arbitrary $f, g \in A$. For the relations existing between the above definition and the classical theory of characters, see [16].

We shall say that a double representation $\{\mathfrak{S}, U, V\}$ is irreducible if the set of operators $U_{f}, V_{g}$ (taken jointly) is irreducible in the usual sense (of course, for double representations on Banach spaces we should distinguish between three kinds of irreducibility, but since we shall be concerned with finite-dimensional double representations such distinctions are useless here).

Now consider on $G$ an indefinitely differentiable function $\phi$ satisfying $\alpha \phi=\phi \alpha$ for every $\alpha \in U^{\circ}(G)$, and invariant under $K$. Let $\mathfrak{B}_{\phi}$ be the set of functions $\alpha \phi, \alpha \in U^{\circ}(G)$. Then for every $\alpha \in U^{\circ}(G)$ we can define on $\mathfrak{B}_{\phi}$ operators $U_{\alpha}: \theta \rightarrow \alpha \theta ; V_{\alpha}: \theta \rightarrow \theta \alpha$; clearly we get a double representation of $U^{\circ}(G)$ on $\mathfrak{B}_{\phi}$.

Theorem 13. Let $\phi$ be a quasi-bounded indefinitely differentiable function on $G$, invariant under $K$; then $\phi$ is proportional to a spherical function if and only if it satisfies the following conditions $\left({ }^{19}\right)$ :

(a) : $\alpha \phi=\phi \alpha$ for every $\alpha \in U^{\circ}(G)$;

(b) : the double representation of $U^{\circ}(G)$ on $\mathfrak{B}_{\phi}$ is irreducible and finitedimensional.

(18) This means that we have $V_{f g}=V_{g} V_{f}$ instead of $V_{f g}=V_{f} V_{g}$.

(19) Condition (a) is equivalent to $\int_{\phi}\left(k g k^{-1} g^{\prime}\right) d k=\int_{\phi}\left(k g^{\prime} k^{-1} g\right) d k$ (under the assumption that $\phi$ is analytic, which is satisfied by spherical functions). 
Proof. The necessity of (a) follows from Lemma 23. To prove the necessity of (b) we may assume that $\phi$ is analytic and that $\phi(\alpha)=\operatorname{Tr}\left(T_{\alpha}\right)$ for some irreducible $p$-dimensional representation $\alpha \rightarrow T_{\alpha}$ of $U^{\circ}(G)$; let $M$ be the set of operators $T_{\alpha} ; M$ is a full matrix algebra and by Lemma 19 there exists a oneto-one correspondence between $M$ and $\mathfrak{B}_{\phi}$, namely $T_{\alpha} \leftrightarrow \alpha^{\prime} \phi$; it is clear that if we identify $\mathfrak{B}_{\phi}$ with $M$, then the double representation of $U^{\circ}(G)$ on $\mathfrak{B}_{\phi}$ leads to a double representation on $M$, constructed in the following way: $U_{\alpha}$ transforms $A \in M$ into $A T_{\alpha^{\prime}}$, and $V_{\alpha}$ transforms $A \in M$ into $T_{\alpha^{\prime}} A$. Therefore, the subspaces of $\mathfrak{B}_{\phi}$ invariant under both the $U_{\alpha}$ and the $V_{\beta}$ correspond to twosided ideals in $M$, and since $\boldsymbol{M}$ is a simple algebra, the irreducibility of the double representation $\left\{\mathfrak{B}_{\phi}, U, V\right\}$ of $U^{\circ}(G)$ is established.

Consider conversely a function $\phi$ satisfying the assumptions of Theorem 13. Since $\mathfrak{B}_{\phi}$ is finite-dimensional, we see as in Lemma 21 that $\phi$ is analytic. Now consider in $\mathfrak{B}_{\phi}$ a minimal subspace $\mathfrak{W}$ invariant under the operators $V_{\alpha}$, and denote by $T_{\alpha}$ the operator induced by $V_{\alpha^{\prime}}$ on $\mathfrak{W}$; clearly $\alpha \rightarrow T_{\alpha}$ is an irreducible representation of $U^{\circ}(G)$ on $\mathfrak{W}$; furthermore, since $U_{\alpha}$ and $V_{\beta}$ commute and since the set of operators $U_{\alpha} V_{\beta}$ is irreducible on $\mathfrak{B}_{\phi}$, it is easy to see that $T_{\alpha}=0$ implies (hence is equivalent to) $V_{\alpha^{\prime}}=0$, hence implies $\phi \alpha^{\prime}=0$, hence implies $\phi(\alpha)=0$; consequently there exists on the set $M$ of operators $T_{\alpha}$ a linear form sp such that $\phi(\alpha)=s p\left(T_{\alpha}\right)$ for every $\alpha \in U^{\circ}(G)$. Now from $\alpha \phi=\phi \alpha$ follows in an obvious way that $\phi(\alpha \beta)=\phi(\beta \alpha)$, so that $s p\left(T_{\alpha} T_{\beta}\right)=s p\left(T_{\beta} T_{\alpha}\right)$; since $M$ is the algebra of all operators on $\mathscr{W}, s p$ is proportional to the ordinary trace on $\boldsymbol{M}$, and we see that $\phi$ satisfies all assumptions of Theorem 12; therefore $\phi$ is proportional to a spherical function.

THEOREM 14. Let $\phi$ be a quasi-bounded function on $G$, invariant under $K$; then $\phi$ is proportional to a spherical function of height one if and only if $\phi$ is an eigenfunction of every differential operator $f \rightarrow \alpha f, \alpha \in U^{\circ}(G)$.

The proof of this theorem is obvious: if $\phi$ is a spherical function of height $p=1$, then $\mathfrak{B}_{\phi}$ has dimension $p^{2}=1$, so that $\alpha \phi$ is proportional to $\phi$ for every $\alpha \in U^{\circ}(G)$; the converse follows from Theorem 13 .

Observe that in the statement of Theorem 14 we did not assume that $\phi$ is analytic, or that $\phi$ is indefinitely differentiable; in fact, even if we know that $\phi$ is a distribution only, then the fact that $\phi$ satisfies elliptic equations will imply that $\phi$ is an analytic function!

Theorem 14 could be proved by using the functional equation of spherical functions of height one instead of Theorem 13. In fact, consider a $\phi$ satisfying

$$
\alpha \phi=\lambda(\alpha) \cdot \phi
$$

for every $\alpha \in U^{\circ}(G)$, and for a given $y \in G$ consider the function

$$
\theta(x)=\int \phi\left(x k y k^{-1}\right) d k=\int \phi\left(k x k^{-1} y\right) d k ;
$$


we obviously have

$$
\alpha \theta(x)=\int \alpha \phi\left(x k y k^{-1}\right) d k=\lambda(\alpha) \theta(x)
$$

for every $\alpha \in U^{\circ}(G)$; comparing (102) and (104) we see that $\theta(\alpha)$ is proportional to $\phi(\alpha)$ for every $\alpha \in U^{\circ}(G)$, hence for every $\alpha \in U(G)$ since both $\phi$ and $\theta$ are invariant under $K$; but $\phi$ and $\theta$ are analytic functions (they satisfy elliptic equations); hence $\theta(x)$ is proportional to $\phi(x)$, and this implies that $\phi$ satisfies, up to a constant factor, the functional equation of spherical functions of height one.

The following theorem (which was never published although it must be known to many people!) is a consequence of Theorem 14.

THEOREM 15. Let $G$ be a compact connected Lie group, and let $\chi$ be a central function on $G$, i.e., $\chi(x y)=\chi(y x)$; then $\chi$ is proportional to a character of $G$ if and only if it is an eigenfunction for every differential operator belonging to the center of $U(G)$.

In fact, if we apply the general theory by taking $K=G$, it is obvious that every spherical function is of height one and reduces to a character of $G$, and conversely; hence to prove Theorem 15 we have only to observe that $U^{\circ}(G)$ reduces to the center of $U(G)$ and to apply Theorem 14 .

Theorem 14 can be used also in the following situation. Let $G$ be a compact connected Lie group and let $K$ be a maximal abelian subgroup of $G$. Let $g \rightarrow T_{g}$ be a (finite-dimensional unitary) irreducible representation of $G$ on a vector space $\mathfrak{S}$; it is well known that there exists at least one character $k \rightarrow \lambda(k)$ of $K$ which is contained exactly once in the given representation of $G$ ( $\lambda$ is the highest weight of the representation); let $a \in \mathfrak{S}$ be a vector belonging to the "weight" $\lambda$; then the function $\theta(g)=\left(T_{g} a, a\right)$ is a spherical function of height one (with respect to the subgroup $K$ ). Consequently we get a method for characterizing these functions by differential equations.

23. Infinitesimal irreducibility of representations. In the preceding sections, we proved that Theorem 8 has an infinitesimal counterpart. We now intend to prove a similar result concerning Theorem 6 of $\$ 2$, namely

THEOREM 16. Let $G$ be a connected Lie group, $K$ a connected compact subgroup of $G, g \rightarrow T_{o}$ a topologically irreducible representation of $G$ on a Banach space $\mathfrak{S}$; assume that $\mathfrak{S}(\mathfrak{b})$ is finite-dimensional for every irreducible representation $\delta$ of $K$, and let $\mathfrak{S}^{\infty}$ be the algebraic direct sum of the various $\mathfrak{S}(\delta)$; then $\mathfrak{S}^{\infty}$ is invariant under every $T_{\alpha}, \alpha \in U(G)$, and the representation $\alpha \rightarrow T_{\alpha}$ of $U(G)$ on $\mathfrak{S}^{\infty}$ is algebraically irreducible.

Of course this result was known to Harish-Chandra for semi-simple groups, but our method works in more general situations. To prove Theorem 16 we shall need some lemmas. 
Lemma 25. For a given vector $a \in \mathfrak{S}$, assume the vectors $T_{k}$ a span a finitedimensional subspace; then $a \in \mathfrak{S}^{\infty}$ and conversely.

Proof. Obvious.

Lemma 26. $\mathfrak{S}^{\infty}$ is invariant under the operators $T_{\alpha}, \alpha \in U(G)$.

Proof. Take an arbitrary vector $a$ in an arbitrary $\mathfrak{S}(\mathfrak{b})$. For every $\alpha \in \boldsymbol{U}(G)$, (105)

$$
T_{k} T_{\alpha} \mathbf{a}=T_{a d(k) \alpha} T_{k} \mathbf{a}
$$

where we set $a d(k) \alpha=\epsilon_{k} \alpha \epsilon_{k^{-1}}$; now it is clear that for a given $\alpha$ the distributions $a d(k) \alpha$ span a finite-dimensional subspace in $U(G)$; since the $T_{k}$ a remain in the finite-dimensional space $\mathfrak{S}(\mathfrak{d})$, we conclude that the $T_{k} T_{\alpha} a$, for a given $\alpha$, span a finite-dimensional subspace of $\mathfrak{S}$; hence

$$
T_{\alpha} a \in \mathfrak{S}^{\infty}
$$

by Lemma 25, and this proves Lemma 26.

LEMma 27. Let a be a nonvanishing vector in $\mathfrak{S}^{\infty}$; then the vectors $T_{\alpha} a$, $\alpha \in U(G)$, are everywhere dense in $\mathfrak{S}$.

Proof. By Hahn-Banach's theorem, we have only to prove that if a vector $\boldsymbol{b} \in \mathfrak{S}^{\prime}$ satisfies $\left(T_{\alpha} \boldsymbol{a}, \boldsymbol{b}\right)=0$ for every $\alpha \in \boldsymbol{U}(G)$, then $\boldsymbol{b}=0$. But if we set $\theta(g)=\left(T_{g} a, b\right)$, we know that $\theta$ is analytic on $G$, and since our assumption means that $\theta(\alpha)=0$ for every $\alpha \in U(G)$, this implies $\theta(g)=0$; hence $\boldsymbol{b}=0$ by the topological irreducibility of the representation $g \rightarrow T_{g}$ of $G$.

REMARK. Of course the above lemma is a special case of a general property of well-behaved vectors; see Theorem 1 in the first note [20] of HarishChandra.

LEMMA 28. Let a be a nonvanishing vector in $\mathfrak{S}^{\infty}$, and denote by $\mathfrak{W}$ the subspace of vectors $T_{\alpha} a, \alpha \in U(G)$; then $\mathfrak{W}$ is invariant under every $E(\mathfrak{b})$.

Proof. Let $\mathfrak{B}$ be the finite-dimensional subspace spanned by the vectors $T_{k} a$; since $K$ is a connected Lie group, the representation $k \rightarrow T_{k}$ on $\mathfrak{B}$ is generated by the corresponding representation $\alpha \rightarrow T_{\alpha}$ of $U(K)$ [of course we may identify $U(K)$ with a subalgebra of $U(G)]$, so that every $T_{k}$ is, on $\mathfrak{B}$, a linear combination of operators $T_{\alpha}, \alpha \in U(K)\left({ }^{20}\right)$; using (105) we see that $\mathfrak{W}$

$\left.{ }^{(20}\right)$ Let $k \rightarrow T_{k}$ be a finite-dimensional irreducible representation of a connected Lie group $K$ on a vector space $\mathfrak{B}$, and consider the corresponding representation $\alpha \rightarrow T_{\alpha}$ of $U(K)$; let $\left(e_{i}\right), 1 \leqq i \leqq n$, be a basis of $\mathfrak{B}$ and $\left(\boldsymbol{e}_{i}^{\prime}\right)$ a basis of the dual vector space $\mathfrak{B}^{\prime}$. Since $T_{k}$ is an analytic function of $k$, every function $\theta_{i j}(k)=\left(T_{k} \boldsymbol{e}_{i}, \mathbf{e}_{j}^{\prime}\right)$ is analytic on $K$, and it is clear that $\theta_{i j}(\boldsymbol{\alpha})$ $=\left(T_{\alpha} \boldsymbol{e}_{i}, \boldsymbol{e}_{j}^{\prime}\right)$ for every $c \in U(K)$. Now take some $k \in K$ such that $k=\exp (\alpha)$ where $\alpha \in U(K)$ belongs to the Lie algebra of $K$; since the functions $\theta_{i j}$ are in finite number we see that if $k$ belongs to a sufficiently small neighborhood of $e$, then we have (Taylor's formula), for arbitrary $i, j$, $\theta_{i j}(k)=\sum_{n \geqq 0} X_{\alpha}^{n} \theta_{i j}(e) / n !$ where we set as usual $X_{\alpha} f=\alpha f$ (convolution product); this means that $\left(T_{k} \mathbf{e}_{i}, \mathbf{e}_{j}^{\prime}\right)=\sum_{n \geqq 0}\left(T_{\alpha}^{n} \mathbf{e}_{i}, \mathbf{e}_{j}^{\prime}\right) / n$ !, so that $T_{k}=\sum_{n \geqq 0} T_{\alpha}^{n} / n$ ! (strongly convergent series); since the set of operators $T_{\alpha}, \alpha \in U(K)$, is a finite-dimensional algebra, we see that this algebra contains $T_{k}$ whenever $k$ is sufficiently close to $e$, hence contains every $T_{k}, k \in K$, by the connectedness of $K$, q.e.d. 
is invariant under $k \rightarrow T_{k}$. Since $\mathfrak{B} \subset \mathfrak{S}^{\infty}$ and since the representation $k \rightarrow T_{k}$ of $K$ on $\mathfrak{S}^{\infty}$ is completely reducible (in the purely algebraic meaning), it is now obvious that $E(\mathfrak{d}) \mathfrak{B}=\mathfrak{W} \cap \mathfrak{S}(\mathfrak{d})$ for every $\mathfrak{d}$.

Proof of Theorem 16. With the notation of Lemma 28 we have to prove that $\mathfrak{B}=\mathfrak{S}^{\infty}$; but since $\mathfrak{B}$ is everywhere dense in $\mathfrak{S}, E(\mathfrak{D}) \mathfrak{W}$ is everywhere dense in $\mathfrak{S}(\mathfrak{D})$ for every $\mathfrak{b}$; since $\mathfrak{S}(\mathfrak{D})$ is finite-dimensional, this proves that $E(\mathfrak{D}) \mathfrak{B}=\mathfrak{S}(\mathfrak{d})$; since $\mathfrak{B}$ is invariant under $E(\mathfrak{d})$, this proves that $\mathfrak{B} \supset \mathfrak{S}(\mathfrak{d})$, so that $\mathfrak{W}=\mathfrak{S}^{\infty}$ and Theorem 16 is entirely proved.

REMARK 12. The representation $\alpha \rightarrow T_{\alpha}$ of $U(G)$ on $\mathfrak{S}^{\infty}$ satisfies the assumptions of Theorem 4 in Harish-Chandra's paper [19], i.e., (a) this representation is algebraically irreducible; (b) the representation $\alpha \rightarrow T_{\alpha}$ of $U(K)$ on $\mathfrak{S}^{\infty}$ is completely reducible (in the algebraic sense); (c) if $\alpha$ belongs to the center $\boldsymbol{Z}(G)$ of $\boldsymbol{U}(G)$, then $T_{\alpha}$ reduces to a scalar on $\mathfrak{S}^{\infty}$ [in fact, $\boldsymbol{Z}(G)$ is contained in the center of $U^{\circ}(G)$; since for every $\delta$ the ring of operators $\widetilde{T}_{\alpha}(\mathfrak{b})$, $\alpha \in U^{\circ}(G)$, is isomorphic to a full matrix algebra, we see that every $\widetilde{T}_{\alpha}(\delta)$, $\alpha \in \boldsymbol{Z}(G)$, reduces to a scalar $c(\alpha, \delta)$; but by Schur's lemma applied to the representation $\alpha \rightarrow T_{\alpha}$ of $U(G)$ on $\mathfrak{S}^{\infty}$ we see that for every number $c$ either $T_{\alpha}=c \cdot 1$ or $T_{\alpha}-c \cdot 1$ has an inverse; this of course implies that $c(\alpha, \delta)$ does not depend on $\delta$, q.e.d.]. Therefore, if $G$ is a complex semi-simple group, we may use the "infinitesimal" results of Harish-Chandra in order to prove "integral" theorems.

For instance, let $g \rightarrow T_{\theta}$ and $g \rightarrow T_{g}^{\prime}$ be two irreducible unitary representations of $G$ on Hilbert spaces $\mathfrak{S}$ and $\mathfrak{S}^{\prime}$, and assume that the corresponding representation $\alpha \rightarrow T_{\alpha}$ and $\alpha \rightarrow T_{\alpha}^{\prime}$ of $U(G)$ on $\mathfrak{S}^{\infty}$ and $\mathfrak{S}^{\prime \infty}$ are algebraically equivalent; then for every $\mathfrak{d}$ the representations $\alpha \rightarrow \widetilde{T}_{\alpha}(\mathfrak{b})$ and $\alpha \rightarrow \widetilde{T}_{\alpha}^{\prime}(\mathfrak{d})$ of $U^{\circ}(G)$ on $\mathfrak{S}(\mathfrak{D})$ and $\mathfrak{S}^{\prime}(\mathfrak{d})$ are algebraically equivalent too, so that we have $\operatorname{Tr}\left[\tilde{T}_{\alpha}(\mathfrak{b})\right]=\operatorname{Tr}\left[\tilde{T}_{\alpha}^{\prime}(\mathfrak{D})\right]$ for every $\alpha \in U^{\circ}(G)$, i.e.,

$$
\phi_{b}(\alpha)=\phi_{\triangleright}^{\prime}(\alpha)
$$

where $\phi_{b}$ and $\phi_{b}^{\prime}$ are the spherical functions of type $\delta$ of the two representations; hence (Lemma 14) $\phi_{\mathfrak{b}}(g)=\phi_{\mathfrak{b}}^{\prime}(g)$ so that the given two representations are unitarily equivalent (see no. 13). Now if $\alpha \in \boldsymbol{Z}(G)$, put $T_{\alpha}=\zeta(\alpha) \cdot 1$ and call $\alpha \rightarrow \zeta(\alpha)$ the infinitesimal character of the representation; then by using Theorem 4 of [19] we see that if $G$ is a complex semi-simple Lie group, then there exists (up to unitary equivalence) only a finite number of irreducible unitary representations of $G$ with a given infinitesimal character and in which a given $\mathrm{b}$ does occur. Of course this result is due to Harish-Chandra; see Theorem 6 in his first note [20].

In the above argument, we asserted that if $\alpha \rightarrow T_{\alpha}$ and $\alpha \rightarrow T_{\alpha}^{\prime}$ are algebraically equivalent representations of $U(G)$, then the representations $\alpha \rightarrow \widetilde{T}_{\alpha}(\mathfrak{l})$ and $\alpha \rightarrow \widetilde{T}_{\alpha}^{\prime}(\mathfrak{D})$ of $U^{\circ}(G)$ on $\mathfrak{S}(\mathfrak{d})$ and $\mathfrak{S}^{\prime}(\mathfrak{D})$ are equivalent too; this requires a proof. Let $k \rightarrow U_{k}$ be an irreducible representation of $K$ belonging to the class $\mathfrak{b}$; let $\alpha \rightarrow U_{\alpha}$ be the corresponding representation of $U(K)$, and 
denote by $\boldsymbol{Z}(K)$ the center of $\boldsymbol{U}(K)$; then $U_{\alpha}$ reduces to a scalar for every $\alpha \in \boldsymbol{Z}(K)$, and it is clear that we have in a more precise way

$$
U_{\alpha}=\operatorname{dim}(\mathfrak{b})^{-2} \cdot \chi_{\mathfrak{b}}(\alpha) \cdot 1=\lambda_{\mathfrak{b}}(\alpha) \cdot 1
$$

where $\chi_{\delta}(k)$ is the character of $\delta$ with the normalization $\chi_{\mathfrak{b}}(e)=\operatorname{dim}(\mathfrak{d})^{2}$; of course $\alpha \rightarrow \lambda_{b}(\alpha)$ is a homomorphism of $\boldsymbol{Z}(K)$ onto the complex field, the infinitesimal character of $b$. It is easy to prove (see [19] and the appendix to [16]) that $\delta$ is uniquely defined by its infinitesimal character when $K$ is connected. Now consider the representation $\alpha \rightarrow T_{\alpha}$ of $U(K)$ on $\mathfrak{S}^{\infty}$; by the above argument we have $T_{\alpha} \boldsymbol{a}=\lambda_{\mathfrak{b}}(\alpha) \cdot \boldsymbol{a}$ for every $\boldsymbol{a} \in \mathfrak{S}(\mathfrak{b})$ and every $\alpha \in \boldsymbol{Z}(K)$; conversely assume that $T_{\alpha} a=\lambda_{b}(\alpha) \cdot a$ for some $a \in \mathfrak{S}^{\infty}$; then we have by the direct property

$$
\sum_{\mathfrak{b}^{\prime}} \lambda_{\mathfrak{b}^{\prime}}(\alpha) E\left(\mathfrak{d}^{\prime}\right) \boldsymbol{a}=\sum_{\mathfrak{b}^{\prime}} \lambda_{\mathfrak{b}}(\alpha) E\left(\boldsymbol{d}^{\prime}\right) \boldsymbol{a},
$$

and since $\mathfrak{b} \neq \mathfrak{d}^{\prime}$ implies $\lambda_{\mathfrak{b}} \neq \lambda_{\mathfrak{b}^{\prime}}$, this obviously implies $a \in \mathfrak{S}(\mathfrak{b})$. In other words, the vectors in $\mathfrak{S}(\delta)$ are characterized by $T_{\alpha} a=\lambda_{\delta}(\alpha) \cdot a$ for every $\alpha$ $\in Z(K)$; this obviously proves that if $a \rightarrow a^{\prime}$ is an isomorphism of $\mathfrak{S}^{\infty}$ onto $\mathfrak{S}^{\prime \infty}$ transforming every $T_{\alpha}$ into the corresponding $T_{\alpha}^{\prime}$, then $a \rightarrow a^{\prime}$ maps $\mathfrak{S}(\boldsymbol{b})$ onto $\mathfrak{S}^{\prime}(\mathfrak{d})$, so that this isomorphism transforms $\widetilde{T}_{\alpha}(\mathfrak{d})$ into $\tilde{T}_{\alpha}^{\prime}(\mathfrak{d})$ for every $\alpha$ $\in U^{\circ}(G)$ as asserted.

\section{APPENDIX}

\section{THE CLASSIFICATION OF FINITE-DIMENSIONAL REPRESENTATIONS OF CLASSICAL GROUPS}

In this appendix we want to discuss a simple method for getting a complete classification of finite-dimensional irreducible representations of the various "classical groups." This method essentially rests upon the following principle (see Lemma 7 in $\$ 1$ ): let $N$ be a connected solvable subgroup of $G$; then every finite-dimensional irreducible representation of $G$ has at least one coefficient $\theta$ satisfying $\theta(n g)=\alpha(n) \theta(g)$ for some character $n \rightarrow \alpha(n)$ of $N$. Of course this principle is not new, and the corresponding "infinitesimal" result has been intensively used by E. Cartan and H. Weyl; but we shall exclusively use "integral" arguments.

In order to simplify the discussion we shall consider the group $G=S L(n, R)$ of all real unimodular $n \times n$ matrices. Our constructions rest upon the existence in $G$ of solvable subgroups which exist in every (complex or real) semisimple Lie group and which have well known interpretations from the infinitesimal point of view (Cartan's subalgebras and roots). These subgroups are important also in the theory of infinite-dimensional irreducible representations, as shown by Gelfand and Naimark.

1. The Lie theorem. Since this theorem is basic in our discussion, we shall prove it by integral methods (which of course do not differ from the infinitesimal ones!). Let $N$ be a solvable group; we denote by $D(N)$ its commutator 
subgroup (generated by the elements $x y x^{-1} y^{-1}$ ) and we define $D^{i}(N)$ $=D\left[D^{i-1}(N)\right]$; the fact that $N$ is solvable is equivalent to $D^{p}(N)=e$ for some $p$; the smallest $p$ is the height of $N$.

LIE'S THEOREM. Let $N$ be a connected topological solvable group; then every finite-dimensional irreducible representation of $N$ is one-dimensional.

Proof. If $N$ has height one, i.e., if $N$ is abelian, the theorem is obvious (Schur's lemma). Assume that $N$ has height $p$ and the theorem is already proved for groups of height $p-1$; then we may use the theorem for the commutator subgroup $\left({ }^{21}\right) Z=D(N)$ of $N$. Let then $n \rightarrow T_{n}$ be a finite-dimensional irreducible representation of $N$ on a vector space ( . Since $(\xi$ is finite-dimensional, the representation $z \rightarrow T_{z}$ of $Z$ contains an irreducible representation of $Z$, so that we can find a character $z \rightarrow \lambda(z)$ and a nonvanishing vector $a_{\lambda} \in \mathbb{E}$ such that

$$
T_{z} a_{\lambda}=\lambda(z) a_{\lambda}
$$

for every $z \in Z$. Denote by $\phi$ the set of characters $\lambda$ of $Z$ such that (1) has a nonvanishing solution $a_{\lambda}$; of course, $\phi$ is a finite set. Now since $Z$ is invariant in $N$, for every character $\lambda$ of $Z$ and every $n \in N$ we can define a character $\lambda_{n}$ of $Z$ by

$$
\lambda_{n}(z)=\lambda\left(n^{-1} z n\right)
$$

from (1) follows

$$
T_{z} T_{n} \mathbf{a}_{\lambda}=T_{n} T_{n}^{-1} T_{z} T_{n} \mathbf{a}_{\lambda}=\lambda_{n}(z) T_{n} \mathbf{a}_{\lambda} ;
$$

hence $\lambda \in \phi$ implies $\lambda_{n} \in \phi$ for every $n \in N$. Now in the set of all characters of $Z$ we can define a natural topology: $\lambda$ converges to $\lambda^{\prime}$ if $\lambda(z)$ converges to $\lambda^{\prime}(z)$ for every $z$; in this topology $\phi$ is a discrete space; on the other hand, it is clear that, for a given $\lambda, \lambda_{n}$ depends continuously on $n \in N$; since $N$ is connected, we see that, for every $\lambda \in \phi$, the set of $\lambda_{n}$ is a connected and finite set; hence $\lambda_{n}=\lambda$ for every $\lambda \in \phi$ and every $n \in N$.

We therefore see that if $\lambda \in \phi$, then the set of vectors $a_{\lambda}$ which satisfy (1) is invariant under the $T_{n}$; since it is a vector subspace of $\mathbb{E}$ and since $\mathbb{E}$ is irreducible under the $T_{n}$ we conclude that

$$
T_{z}=\lambda(z) \cdot 1
$$

for every $z \in Z$ (so that $\phi$ contains one character of $Z$ ).

Now take an arbitrary $n_{0} \in N$ and choose an eigenvector $a_{0}$ of $n_{0}$;

(21) Of course we should prove that the commutator group $Z$ of a connected group $N$ is itself connected; but this is easy (first, the set of elements $x y x^{-1} y^{-1}$ is the map of $N \times N$ under the continuous mapping $(x, y) \rightarrow x y x^{-1} y^{-1}$ of $N \times N$ into $N$; hence this set $S$ is connected; now denote by $S_{n}$ the set of products $x_{1} \cdots x_{n}$, where every $x_{i}$ belongs to $S$; by a similar argument we see that every $S_{n}$ is connected; since $S_{n} \subset S_{n+1}$ and since $Z$ is the union of the $S_{n}$, we see that $Z$ is connected). 


$$
T_{n_{0}} \mathbf{a}_{0}=c \cdot \mathbf{a}_{0}
$$

for every $n$, we have $n_{0}^{-1} n^{-1} n_{0} n \in Z$ so that by (4)

$$
T_{n_{0}} T_{n}=\lambda\left(n_{0}^{-1} n^{-1} n_{0} n\right) T_{n} T_{n_{0}} ;
$$

this and (5) prove that for every $n \in N, T_{n} \mathbf{a}_{0}$ is an eigenvector of $T_{n_{0}}$; furthermore the corresponding eigenvalue $c \cdot \lambda\left(n_{0}^{-1} n^{-1} n_{0} n\right)$ depends continuously on $n$, hence, by the connectedness of $N$, does not depend on $n$. Therefore the set of eigenvectors satisfying (5) for given $c$ and $n_{0}$ is invariant under $T_{n}$; by the irreducibility, this proves that $T_{n_{0}}$ reduces to a scalar, and this concludes the proof of Lie's theorem.

2. Upper and lower weights of a representation of $G$. We shall consider, in the group $G=S L(n, R)$, the following subgroups:

the subgroup $H$ of diagonal matrices $h=\left(h_{1}, \cdots, h_{n}\right)$;

the subgroup $P$ of upper triangular matrices $p=\left(p_{i j}\right)$, with

$$
p_{i j}=0 \text { for } i>j \text {; }
$$

the subgroup $X$ of matrices $x \in P$ such that $x_{i i}=1,1 \leqq i \leqq n$;

the subgroup $N$ of lower triangular matrices $n=\left(n_{i j}\right)$ with

$$
n_{i j}=0 \text { for } i<j ;
$$

the subgroup $Y$ of matrices $y \in N$ such that $y_{i i}=1,1 \leqq i \leqq n$.

It is clear that $P$ (resp. $N$ ) is a solvable connected group whose commutator subgroup is $X$ (resp. $Y$ ).

Lemma 1. Let $g \in G$; define $g_{0}=1$ and

$$
g_{i}=\left|\begin{array}{c}
g_{11} \cdots g_{1 i} \\
\cdots \cdots \\
g_{i 1} \cdots g_{i i}
\end{array}\right|, \quad 1 \leqq i \leqq n,
$$

and assume that $g_{i} \neq 0,1 \leqq i \leqq n$; then $g$ has a unique representation $g=y \cdot h \cdot x$; furthermore

$$
h_{i}=g_{i} / g_{i-1} .
$$

We leave the proof to the reader.

Now consider an irreducible representation $g \rightarrow T_{g}$ of $G$ on a finite-dimensional vector space $\mathfrak{S}$ over the field of complex numbers. By Lie's theorem, there exists at least one character $p \rightarrow \lambda(p)$ of $P$ such that

$$
T_{p} \mathbf{a}=\lambda(p) \boldsymbol{a}
$$

for some nonvanishing vector $a \in \mathfrak{S}$; we shall say that $\lambda$ is the upper weight of the representation. 
Lemma 2. The upper weight $\lambda$ is unique; the solutions a of (8) form a onedimensional subspace of $\mathfrak{S}$; there exists at most one irreducible representation with a given upper weight.

To prove Lemma 2, consider the contragredient representation of $G$ on the vector space $\mathfrak{S}^{\prime}$ dual to $\mathfrak{S}$. Using Lie's theorem we see that there exists a character $n \rightarrow \mu(n)$ of $N$ and a nonvanishing vector $b \in \mathfrak{S}^{\prime}$ such that

$$
{ }^{t} T_{n} \boldsymbol{b}=\mu(n) \boldsymbol{b} ;
$$

now consider the coefficient

$$
\theta(g)=\left(T_{g} a, b\right)
$$

of the given representation; using (8) and (9) and the fact that $\lambda(x)=\mu(y)=1$ (this is due to the fact that $X$ and $Y$ are the commutator subgroups of $P$ and $N)$ we find at once

$$
\theta(y h x)=\lambda(h) \theta(e)=\mu(h) \theta(e) ;
$$

hence $\theta(e)=0$ would imply $\theta(g)=0$ for an everywhere dense set of $g$ (Lemma 1 ), hence by the continuity of $\theta$ would prove that $b$ is orthogonal to all vectors $T_{g} a$, which is impossible by the irreducibility of the representation and $b \neq 0$; hence $\theta(e) \neq 0$, so that (11) proves that $\lambda(h)=\mu(h)$; hence $\lambda$ is unique. Furthermore, for a fixed $b$ satisfying (9), relation (11) is equivalent to the fact that $\boldsymbol{a}$ satisfies (8), for (11) implies $\theta(g p)=\lambda(p) \theta(g)$, i.e., $\left(T_{p} \boldsymbol{a},{ }^{t} T_{\theta} \boldsymbol{b}\right)$ $=\lambda(p) \cdot\left(a,{ }^{t} T_{g} b\right)$, and since the vectors ${ }^{t} T_{g} b, g \in G$, span $\mathfrak{S}^{\prime}$, our assertion is proved. But it is clear by Lemma 1 that the space of functions $\theta$ satisfying (11) is one-dimensional; since the correspondence between the vector $a$ and the corresponding function $\theta$ is one-to-one, we see that $\boldsymbol{a}$ is unique up to a constant factor. Finally, if $\lambda$ is given, then by (11) and Lemma 1 we know a coefficient of the given representation, so that this irreducible representation is unique up to equivalence.

Of course, instead of using the upper weight $\lambda$, we could use the lower weight, i.e., the character $n \rightarrow \lambda^{\prime}(n)$ of $N$ such that equation

$$
T_{n} \boldsymbol{a}^{\prime}=\lambda^{\prime}(n) \boldsymbol{a}^{\prime}
$$

has at least one nonvanishing solution $a^{\prime}$.

3. Determination of all possible upper weights. Let $h \rightarrow \lambda(h)$ be a character of $H$. If $\lambda$ is the restriction to $H$ of the upper weight of some irreducible finite-dimensional representation of $G$, then by the above results there exists on $G$ a continuous function $\theta(g)$ which satisfies

$$
\theta(y h x)=\lambda(h)
$$

for arbitrary $x, h, y$. Setting

$$
\lambda(h)=\left|h_{1}\right|^{\rho_{1}} h_{1}^{\epsilon_{1}} \cdots\left|h_{n-1}\right|^{\rho_{n-1}} h_{n-1}^{\epsilon_{n-1}}
$$


(we recall that $H$ is isomorphic to $R_{*}^{n-1}$, where $R_{*}$ is the multiplicative group of nonvanishing real numbers) with arbitrary complex parameters $\rho_{1}, \cdots, \rho_{n-1}$ and with $\epsilon_{i}=0$ or 1 , we see by (7) that if every $g_{i}$ is $\neq 0$, then

$$
\theta(g)=\prod_{i=1}^{n-1}\left|g_{i}\right|^{p_{i}} \cdot g_{i}^{\eta_{i}}
$$

where

$$
\begin{aligned}
& \nu_{1}=\rho_{1}-\rho_{2} ; \cdots ; \nu_{n-2}=\rho_{n-2}-\rho_{n-1} ; \nu_{n-1}=\rho_{n-1}, \\
& \eta_{1}=\epsilon_{1}-\epsilon_{2} ; \cdots ; \eta_{n-2}=\epsilon_{n-2}-\epsilon_{n-1} ; \eta_{n-1}=\epsilon_{n-1} .
\end{aligned}
$$

To determine whether (14) corresponds to a finite-dimensional irreducible representation of $G$, we observe that such a representation is not only continuous but also analytic with respect to the parameters of $G$, so that (15) must be an analytic function on $G$.

Lemma 3. Assume there exists on $G$ an analytic function satisfying (15) when all $g_{i}$ are $\neq 0$; then $\nu_{i}$ is an even integer satisfying $\nu_{i}+\eta_{i} \geqq 0,1 \leqq i \leqq n-1$, and conversely.

To prove Lemma 3 we first observe that $S L(n, R)$ contains a subgroup isomorphic with $S L(n-1, R)$, namely the group of those $g \in G$ such that $g_{n i}=g_{i n}=\delta_{i n}$, and a subgroup isomorphic to $S L(2, R)$, namely the group of matrices

$$
\left(\begin{array}{ccccc}
1 & 0 & \cdots & 0 & 0 \\
0 & 1 & \cdots & 0 & 0 \\
\cdot & \cdot & \cdot & \cdot & \cdot \\
0 & 0 & \cdots & a & b \\
0 & 0 & \cdots & c & d
\end{array}\right)
$$

so that we see by induction on $n$ that we have to prove Lemma 3 in case $n=2$ only. But if we consider in $S L(2, R)$ the subset of matrices

$$
\left(\begin{array}{rr}
x & 1 \\
-1 & 0
\end{array}\right)
$$

and if we set $\nu_{1}=\nu, \eta_{1}=\eta$, we have to express that there exists on the line $R$ an analytic function $\theta(x)$ such that $\theta(x)=|x|^{\nu} \cdot x^{\nu}$ for $x \neq 0$; if we write that $\theta$ has a power series expansion in a neighborhood of $x=0$, we find at once the conditions $\nu=2 p, \nu+\eta \geqq 0$, so that the necessity of the conditions stated in Lemma 3 is established; the fact that they are sufficient is obvious.

Lemma 3 yields necessary conditions for (14) to be the upper weight of a finite-dimensional representation of $G$. By (17) and the fact that $\epsilon_{i}=0$ or 1 , we see that $\eta_{i}=1$ or 0 or -1 ; since $\nu_{i}$ must be an even integer and since 
$\nu_{i}+\eta_{i}$ must be $\geqq 0$, we see that every $\nu_{i}$ must be $\geqq 0$; since $\rho_{n-1}=\nu_{n-1}$ must be an even integer, we already see that we may write

$$
\rho_{i}=2 p_{i}
$$

where the $p_{i}$ are integers satisfying

$$
p_{1} \geqq p_{2} \geqq \cdots \geqq p_{n-1} \geqq 0 .
$$

Furthermore, by (18), we may write

$$
\lambda(h)=h_{1}^{r_{1}} \cdots h_{n-1}^{r_{n-1}}
$$

where

$$
r_{i}=\rho_{i}+\epsilon_{i},
$$

and since $\nu_{i}+\eta_{i}=r_{i}-r_{i+1}$, we see that

$$
r_{1} \geqq r_{2} \geqq \cdots \geqq r_{n-1} \geqq 0 ;
$$

conversely it is clear that every function (20) where the exponents satisfy (22) can be written in the form (14) in such a way that the conditions of Lemma 3 are satisfied.

Lemma 4. Let $r_{1}, \cdots, r_{n-1}$ be integers satisfying (22); then there exists one and only one finite-dimensional irreducible representation of $G$ whose upper weight is the function (20).

To prove Lemma 4, consider the function

$$
\theta(g)=\prod_{i=1}^{n-1} g_{i}^{\nu_{i}}
$$

where $\nu_{1}=r_{1}-r_{2}, \cdots, \nu_{n-1}=r_{n-1}$, and denote by $\mathfrak{B}$ the vector space spanned by the right translates of $\theta$; define a representation $g \rightarrow T_{\theta}$ of $G$ on $\mathfrak{B}$ by $T_{\theta} \phi\left(g^{\prime}\right)$ $=\phi\left(g^{\prime} g\right), \phi \in \mathfrak{B}$. First of all, (23) proves that $\theta$ is a polynomial with respect to the $g_{i j}$, so that $\mathfrak{B}$ is a set of polynomials whose degree is bounded; hence $\mathfrak{B}$ is finte-dimensional $\left({ }^{22}\right)$. Furthermore it is clear by the very construction of $\theta$ that

$$
T_{p} \theta=\lambda(p) \theta
$$

where $\lambda(h)$ is the function (20). Therefore Lemma 4 will be proved if we establish that the representation $g \rightarrow T_{g}$ is irreducible. But assume that $\mathfrak{B}$ decomposes into the direct sum of two invariant subspaces $\mathfrak{B}_{1}$ and $\mathfrak{B}_{2}$, and denote by $\theta_{1}$ and $\theta_{2}$ the components of $\theta$ on $\mathfrak{W}_{1}$ and $\mathfrak{W}_{2}$; by (24) it is obvious

(22) If $G$ were an arbitrary semi-simple group, this fact probably would require a nontrivial proof; it is interesting to observe that a similar problem arises in Harish-Chandra's method (see Lemma 15 in Part I of [19]). 
that every $\theta_{i}$ satisfies $T_{p} \theta_{i}=\lambda(p) \theta_{i}$; on the other hand it is clear that $\theta$ satisfies $\theta(y g)=\theta(g)$, and since right translations preserve this relation, we see that it holds for every $\phi \in \mathfrak{B}$; in particular we see that every $\theta_{i}$ satisfies $\theta_{i}(y h x)$ $=\lambda(h) \theta_{i}(e)$; hence by Lemma 1 every $\theta_{i}$ is proportional to $\theta$, which of course proves the irreducibility.

In conclusion, we have obtained a complete classification of all finitedimensional irreducible representations of $S L(n, R)$ : every such representation is characterized by integers $r_{1}, \cdots, r_{n-1}$ satisfying (22), and to every solution of (22) corresponds such a representation, whose upper weight is given by (20). Of course this result is not new.

4. A remark on $S L(n, C)$. The above method obviously applies to $S L(n, C)$ (and more generally to all classical groups), and it leads to an integral formula for the characters of $S L(n, C)$ which seems to be new.

In fact, let $g \rightarrow T_{g}$ be a complex-analytic finite-dimensional irreducible representation of $G=S L(n, C)$ on a vector space $\mathfrak{S}$. By using as in no. 2 subgroups $H, P, N, X, Y$ we see that the given representation has a coefficient $\theta$ satisfying $\theta(y h x)=\lambda(h)$ for some character of $H$; but since we are dealing with complex-analytic representations, $\lambda(h)$ must be a complex-analytic character of $H$, so that $\lambda(h)=h_{1}^{r_{1}} \ldots h_{n-1}^{r_{n-1}}$ with integers $r_{i}$; using arguments similar to the proof of Lemmas 3 and 4 we see that $r_{1}, \cdots, r_{n-1}$ correspond to a complex-analytic representation of $G$ if and only if $r_{1} \geqq r_{2} \geqq \cdots \cdot r_{n-1} \geqq 0$. Hence

$$
\theta(g)=g_{1}^{\nu_{1}} \cdots g_{n-1}^{\nu_{n-1}}
$$

where $\nu_{1}=r_{1}-r_{2}, \cdots, \nu_{n-1}=r_{n-1}$ are positive integers. Now denote by $K$ a maximal compact subgroup of $G$, e.g., the unitary subgroup $S U(n)$. Since $g \rightarrow T_{g}$ is analytic with respect to the complex parameters on $G$, it is clear by the unitary trick that $k \rightarrow T_{k}$ is an irreducible representation of $K$. But it is known that if $k \rightarrow T_{k}$ is an irreducible representation of a compact group $K$ on a vector space $\mathfrak{S}$, then for every operator $A$ on $\mathfrak{S}$ we have

$$
\operatorname{dim}(\mathfrak{S}) \cdot \int T_{k} A T_{k}^{-1} \cdot d k=\operatorname{Tr}(A) \cdot 1
$$

from this and (25) we conclude: the character $\chi(g)$ of the given representation of $G$ is the function

$$
\chi(g)=\operatorname{dim}(\mathfrak{S}) \cdot \int \theta\left(k g k^{-1}\right) d k
$$

where the function $\theta$ is given by (25).

The complete calculation of integrals such as (26) is unfortunately a nontrivial problem-but this problem can be solved by using a method due to Gelfand and Naimark (see pp. 68-77 of [12] where integrals similar to (26) arise in connection with spherical functions). The case $n=2$ however is an 
exception; setting

$$
g=\left(\begin{array}{ll}
x & y \\
z & t
\end{array}\right) ; \quad k=\left(\begin{array}{rr}
u & v \\
-\bar{v} & \bar{u}
\end{array}\right), \quad u \bar{u}+v \bar{v}=1,
$$

we find at once $\left(k g k^{-1}\right)_{1}=\bar{u}(u x+v z)+\bar{v}(u y+v t)$; hence if $g$ is a diagonal matrix:

$$
g=\left(\begin{array}{ll}
\lambda & 0 \\
0 & \lambda^{-1}
\end{array}\right)
$$

we get

$$
\theta(g)=\left(u \bar{u} \lambda+v \bar{v} \lambda^{-1}\right)^{\nu} ;
$$

setting $u=e^{2 \pi i \alpha} \cos \phi, v=e^{2 \pi i \beta} \sin \phi$, so that $d k=\sin \phi \cos \phi d \alpha d \beta d \phi$, we see that

$$
\chi(g)=(2 \pi)^{-1} \int_{0}^{2 \pi}\left(\lambda \cos ^{2} \phi+\lambda^{-1} \sin ^{2} \phi\right)^{\nu} \sin \phi \cos \phi d \phi
$$

up to a constant factor, from which the classical result follows, namely that

$$
\chi(g)=\frac{\lambda^{\nu+1}-\lambda^{-\nu-1}}{(\nu+1)\left(\lambda-\lambda^{-1}\right)}
$$

up to a constant factor.

It should be observed that the above method does not directly lead to the known formulas for the dimensions of the irreducible representations, while $\mathrm{H}$. Weyl's method does. Actually the dimension of the representation whose character is (27) is given by

$$
\left(\int|\chi(k)|^{2} d k\right)^{-1 / 2}
$$

provided $\chi$ is normalized by $\chi(e)=1$ and provided $d k$ is chosen (as done through this paper) in such a way that $\int d k=1$. Although (28) can be computed in an elementary way on $S U(2)$, the calculation of such integrals on $S U(n)$, arbitrary $n$, requires the integration formulas due to $\mathrm{H}$. Weyl.

\section{REFERENCES}

1. V. Bargman, Irreducible unitary representations of the Lorentz group, Ann. of Math. vol. 48 (1947) pp. 568-640.

2. N. Bourbaki, Topologie générale, Chap. X, Paris, 1949.

3. C. Chevalley, Theory of Lie groups, I, Princeton, 1946.

4. J. Dieudonné, La dualité dans les espaces vectoriels topologiques, Ann. École Norm. vol. 54 (1941) pp. 107-139.

5. L. Gårding, Note on continuous representations of Lie groups, Proc. Nat. Acad. Sci. U.S.A. vol. 33 (1947) pp. 331-332.

6. I. Gelfand, Spherical functions on symmetric Riemann spaces, Doklady Akad. Nauk. SSSR. vol. 70 (1950) pp. 5-8. 
7. I. Gelfand and M. Naimark, The principal series of irreducible representations of the complex unimodular group, Doklady Acad. Nauk. SSSR. vol. 56 (1947) pp. 3-4.

8. - Unitary representations of semi-simple Lie groups, I, Mat. Sbornik N.S. vol. 21 (1947) pp. 405-434.

9. - Unitary representations of the Lorentz group, Izvestiya Akad. Nauk SSSR. vol. 11 (1947) pp. 411-504.

10. - On the connexion between the representations of complex semi-simple Lie groups and their maximal compact subgroups, Doklady Acad. Nauk SSSR. vol. 63 (1948) pp. 225-228.

11. - Connexions between unitary representations of the complex unimodular group and its unitary subgroup, Izvestiya Akad. Nauk SSSR; vol. 14 (1950) pp. 239-260.

12. - Unitary representations of the classical groups, Trudy Matematičeskogo Instituta Imeni V. A. Steklova vol. 36 (1950) p. 288.

13. R. Godement, Les fonctions de type positif et la théorie des groupes, Trans. Amer. Math. Soc. vol. 63 (1948) pp. 1-84.

14. —— Thêorèmes taubêriens et thêorie spectrale, Ann. École Norm. vol. 64 (1948) pp. 119-138.

15. - Sur la théorie des représentations unitaires, Ann. of Math. vol. 53 (1951) pp. 68-124.

16. - Mémorie sur la théorie des caractères dans les groupes localement compacts unimodulaires, J. Math. Pures Appl. vol. 30 (1951) pp. 1-110.

17. Harish-Chandra, In finite irreducible representations of the Lorentz group, Proc. Royal Soc. London, Ser. A vol. 189 (1947) pp. 372-401.

299-330.

18. - Lie algebras and the Tannaka duality theorem, Ann. of Math. vol. 51 (1950) pp.

19. - On some applications of the universal enveloping algebra of a semi-simple Lie algebra, Trans. Amer. Math. Soc. vol. 70 (1951) pp. 28-96.

20. —, Representations of semi-simple Lie groups, Proc. Nat. Acad. Sci. U.S.A. vol. 37, pp. 170-173, 362-365, 366-369). 558.

21. K. Iwasawa, On some types of topological groups, Ann. of Math. vol. 50 (1949) pp. 507-

22. N. Jacobson, Structure theory of simple rings without finiteness assumptions, Trans. Amer. Math. Soc. vol. 57 (1945) pp. 228-245.

23. I. Kaplansky, Groups with representations of bounded degree, Canadian Journal of Mathematics vol. 1 (1949) pp. 105-112.

24. - The structure of certain operator algebras, Trans. Amer. Math. Soc. vol. 70 (1951) pp. 219-255.

25. G. W. Mackey, Imprimitivity for representations of locally compact groups, Proc. Nat. Acad. Sci. U.S.A. vol. 35 (1949) pp. 537-545.

26. M. Naimark, Normed rings with an involution, Uspehi Matematičeskih Nauk III, no. 5 (1948) pp. 52-145.

27. J. von Neumann, Zur algebra der Funktionaloperatoren und Theorie der normalen Operatoren, Math. Ann. vol. 102 (1929) pp. 370-427.

28. L. Schwartz, Theorie des Distributions I, II, Paris, 1950, 1951.

29. A. Weil, L'intégration dans les groupes topologiques et ses applications, Paris, 1940.

30. H. Weyl, Gruppen theorie und Quantenmechanik, 2d ed., Leipzig, 1931.

UNIVERSITY OF NANCY,

Nancy, France. 